\title{
Hydrogeologic and Water-Quality Data for the Explosive Experimental Area, Naval Surface Warfare Center, Dahlgren Site, Dahlgren, Virginia
}

By Emily C. Hammond and Clifton F. Bell

U.S. GEOLOGICAL SURVEY

Open-File Report 95-386

Prepared in cooperation with the

SAFETY AND ENVIRONMENTAL OFFICE, NAVAL SURFACE WARFARE CENTER, DAHLGREN SITE

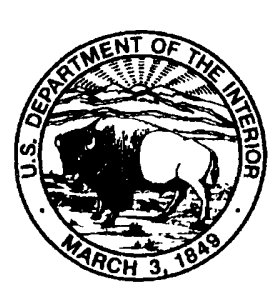

Richmond, Virginia 


\title{
U.S. DEPARTMENT OF THE INTERIOR \\ BRUCE BABBITT, Secretary
}

\author{
U.S. GEOLOGICAL SURVEY \\ Gordon P. Eaton, Director
}

For additional information write to:

District Chief

U.S. Geological Survey

3600 West Broad Street

Room 606

Richmond, VA 23230
Copies of this report can be purchased from:

U.S. Geological Survey

Earth Science Information Center

Open-File Reports Section

Box 25286, MS 517

Denver Federal Center

Denver, CO 80225 


\section{CONTENTS}

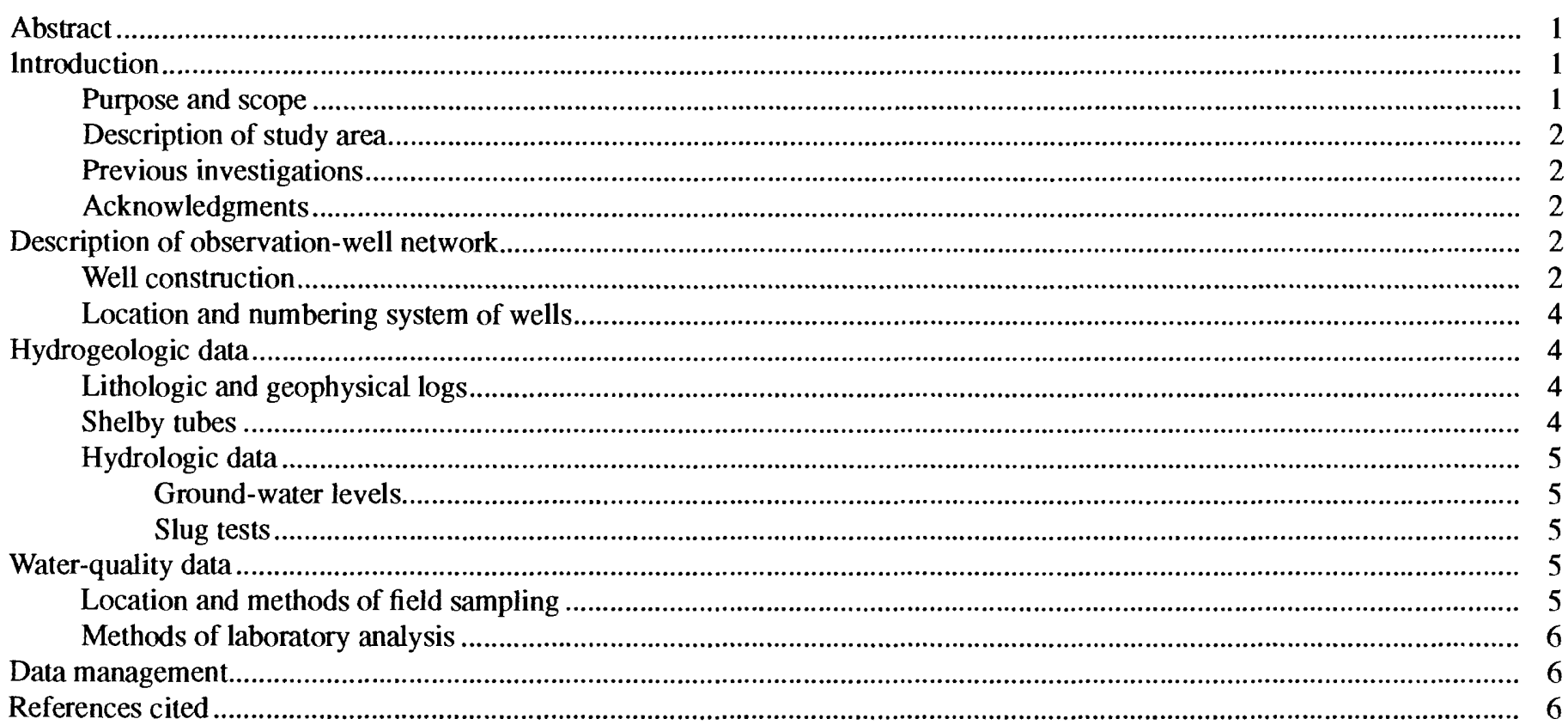

PLATE

1. Map showing locations of observation wells and surface-water sampling sites in the study area In pocket

\section{FIGURES}

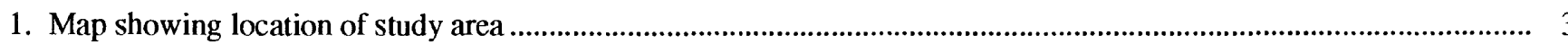

2,3. Schematic diagrams showing:

2. Geologic and hydrogeologic units of the shallow aquifer system. .......................................................................... 36

3. Standard construction of wells completed in a $(A)$ confined aquifer and $(B)$ unconfined aquifer ............................... 37

4. Graphs showing natural gamma-radiation logs of observation wells in the Columbia aquifer........................................ 38

5-12. Graphs showing selected geophysical logs of observation wells ........................................................................ 42

13-22. Hydrographs showing minimum daily water levels for selected observation wells...................................................... 50

23-49. Graphs showing rising-head and falling-head data for slug-test data of observation wells............................................ 55

\section{TABLES}

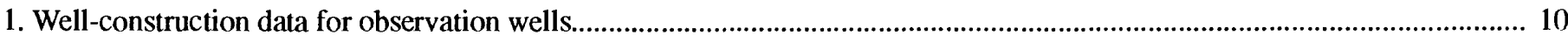

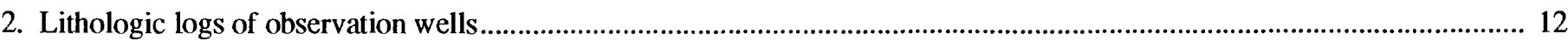

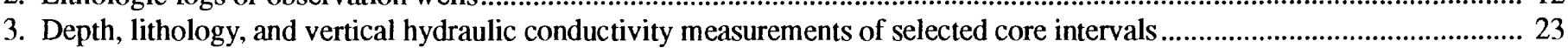

4. Relative percentage of abundance of minerals in selected core intervals............................................................................. 24

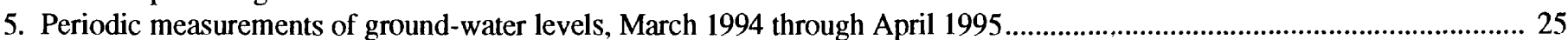

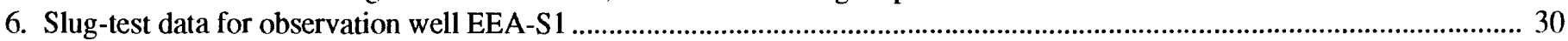

7. Reporting units and limits for selected water-quality constituents ................................................................................... 31

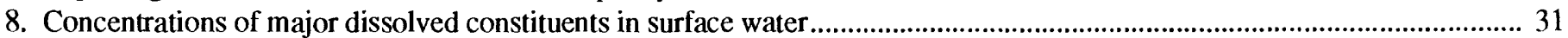

9. Concentrations of major dissolved constituents in ground water ..................................................................................... 32 


\section{CONVERSION FACTORS, VERTICAL DATUM, ABBREVIATED WATER-QUALITY UNITS, AND TRADE-NAME DISCLAIMER}

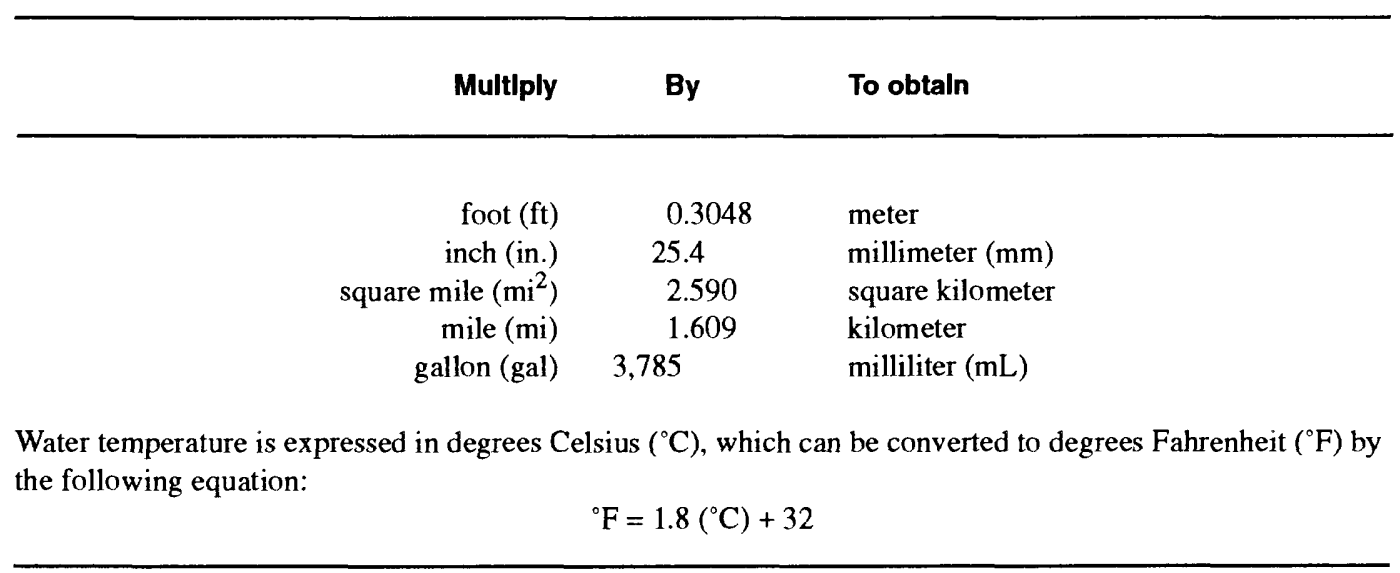

Sea level: In this report "sea level" refers to the National Geodetic Vertical Datum of 1929-a geodetic datum derived from a general adjustment of the first-order level nets of the United States and Canada, formerly called Sea Level Datum of 1929.

Abbreviated water-quality units: Chemical concentration and specific electrical conductance in this report are expressed in metric units. Chemical concentration is expressed in milligrams per liter $(\mathrm{mg} / \mathrm{L})$ or in mocrograms per liter $(\mu \mathrm{g} / \mathrm{L})$. Specific electrical conductance of water is expressed in microsiemens per centimeter at 25 degrees Celsius $(\mu \mathrm{S} / \mathrm{cm})$.

Trade-name disclalmer: Any use of trade or product names in this report is for identification purposes only, and does not constitute endorsement by the U.S. Geological Survey, nor impute responsibility for any existing or potential resources. 


\title{
Hydrogeologic and Water-Quality Data for the Explosive Experimental Area, Naval Surface Warfare Center, Dahlgren Site, Dahlgren, Virginia
}

\author{
By Emily C. Hammond and Clifton F. Bell
}

\begin{abstract}
Hydrogeologic and water-quality data were collected at the Explosive Experimental Area, Naval Surface Warfare Center, Dahlgren Site at Dahlgren, Virginia, as part of a hydrogeologic assessment of the shallow aquifer system begun in 1993. The U.S. Geological Survey conducted this study to provide the U.S. Navy with hydrogeologic data to aid in the evaluation of the effects from remediation of contaminated sites and to protect against additional contamination. This report describes the groundwater observation-well network, hydrogeologic, and water-quality data collected between October 1993 and April 1995. The report includes a description of the locations and construction of 28 observation wells on the Explosive Experimental Area. Hydrogeologic data include lithologic logs, geophysical logs, and vertical hydraulic conductivity measurements of selected core intervals. Hydrologic data include synoptic and hourly measurements of ground-water levels, and observation-well slug tests to determine horizontal hydraulic conductivity. Water-quality data include analyses of major dissolved constituents in ground water and surface water.
\end{abstract}

\section{INTRODUCTION}

The Naval Surface Warfare Center, Dahlgren Site (NSWCDL) at Dahlgren, Va., was established in 1918 as a proving ground for naval ordnance (fig. 1). Today the NSWCDL Mainside also designs both offensive and defensive naval surface warfare systems, including computer models for missile flight and artificial satellite motion. The Explosive Experimental Area (EEA), which is south of Machodoc Creek, is used for testing naval ordnance. In the past, activities conducted at the NSWCDL have had the potential to contaminate ground water, soil, and (or) surface water. The U.S.

Environmental Protection Agency placed the NSWCDL on the National Priority List (NPL) in February 1992, and categorized six sites on the EEA as Solid Waste Management Units (SWMU's) (Halliburton NUS, 1995). SWMU's are areas where solid wastes have been systematically released, even if the areas were not intended for the management of solid or hazardous wastes.

In 1993, the U. S. Geological Survey (USGS), in cooperation with the NSWCDL, began an investigation of the shallow aquifer system underlying the EEA. The USGS conducted this study to provide the U.S. Navy with information to assist in further NPL activities. The study was designed to provide basic hydrogeologic and water-quality data for describing the natural properties and characteristics of the shallow aquifer system. Characterization of individual SWMU's was not an objective of this study.

\section{Purpose and Scope}

The purpose of this report is to present hydrogeologic and general water-quality data collected at the EEA of the NSWCDL, Dahlgren, Va., between October 1993 and April 1995. The report includes (1) descriptions of the observation-well network, (2) hydrogeologic data, and (3) water-quality data. The hydrogeologic and water-quality data are presented in figures and tables.

Twenty-eight observation wells were installed at 20 locations on the EEA of the NSWCDL. Lithologic descriptions and geophysical logs from the 28 observation wells were recorded. Selected intervals were cored 
for analysis of mineralogic characteristics and vertical hydraulic conductivity. Data are presented from slug tests of 27 observation wells. These slug tests were subsequently used to estimate horizontal hydraulic conductivity. Synoptic water levels were measured periodically in all of the wells. Hourly water levels were recorded by analog-to-digital recorders (ADR's) on 10 wells. Water samples were collected from all 28 observation wells and from 3 surface-water sampling sites. The water samples were analyzed for selected constituents to establish background water-quality conditions.

\section{Description of Study Area}

The NSWCDL is located in King George County, Va., and is bounded on the east by the Potomac River (fig. 1). The topography at NSWCDL is generally flat, with a maximum relief of about $20 \mathrm{ft}$ over most of the site. The land use on the installation is forest, open field, marsh, and military operations facilities. Upper Machodoc Creek divides the installation into two areas: the Mainside to the north, which is approximately 2,678 acres, and the EEA to the south, which is approximately 1,614 acres (pl. 1). On the EEA, surface-water bodies include Black Marsh and several unnamed tributaries to Upper Machodoc Creek.

Unconsolidated sedimentary deposits of the Atlantic Coastal Plain underlie the NSWCDL. These deposits consist of tabular bodies of interbedded sand, silt, and clay, and range in age from Cretaceous to Quatemary (Meng and Harsh, 1988). Deposits of the Tabb Formation of Pleistocene age crop out at the surface of the installation (Mixon and others, 1989). At some locations on the EEA, the surficial material is fill.

The shallow aquifer system underlying the NSWCDL (fig. 2), as defined in this report, consists of the Columbia aquifer, which is usually unconfined, and a confined aquifer (unpublished data on file in the Virginia District office of the U.S. Geological Survey). The Columbia aquifer is underlain by the upper confining unit, which overlies the upper confined aquifer. The upper confined aquifer is underlain by the NanjemoyMarlboro confining unit. In some areas of the EEA, the upper confining unit and upper confined aquifer are missing and the Columbia aquifer lies directly on the Nanjemoy-Marlboro confining unit (unpublished data on file in the Virginia District office of the U.S. Geological Survey).

\section{Previous Investigations}

An Initial Assessment Study was performed at the NSWCDL in 1981 (Fred C. Hart Associates, 1983), which identified 36 potentially contaminated sites on the NSWCDL. Of the 36 potentially contaminated sites, 10 sites are located on the EEA; however, Confirmation Studies were not recommended for any of these sites (O'Brien and Gere, 1986). A final Site Management Plan was developed in 1995 (Halliburton NUS, 1995) that designated 2 of the 10 EEA sites as SWMU's, and provides estimated schedules for conducting Comprehensive Environmental Response, Compensation, and Liability Act activities. The USGS began a hydrogeologic framework investigation of the Mainside in 1992. Hydrogeologic and water-quality data collected for the study are presented in a data report (Bell and others, 1994) and in unpublished data on file in the Virginia District office of the U.S. Geological Survey.

\section{Acknowledgments}

The authors wish to thank Ann Swope, Billie Weedon, Collins Johnson, and other members of the Environmental Division, NSWCDL, for their assistance in project planning and implementation. James DeShazo, facilities manager, and Robert Hoyer assisted in coordinating activities at the EEA. The U.S. Army Corps of Engineers (COE) completed the drilling operations at the EEA in a safe and efficient manner under the supervision of Charles Brown.

\section{DESCRIPTION OF OBSERVATION-WELL NETWORK}

Observation wells were sited to obtain maximum areal coverage of the EEA study area, to have drill sites accessible by drilling equipment, and to avoid areas of known or suspected contamination. Observation well locations are shown on plate 1 .

\section{Well Construction}

All 28 observation wells installed at the NSWCDL for the hydrogeologic assessment were constructed of 4-in. inside-diameter polyvinyl chloride (PVC) casing. Twenty wells were screened in the Columbia aquifer, of which 17 wells were augered and 3 wells (wells EEA-S6, 


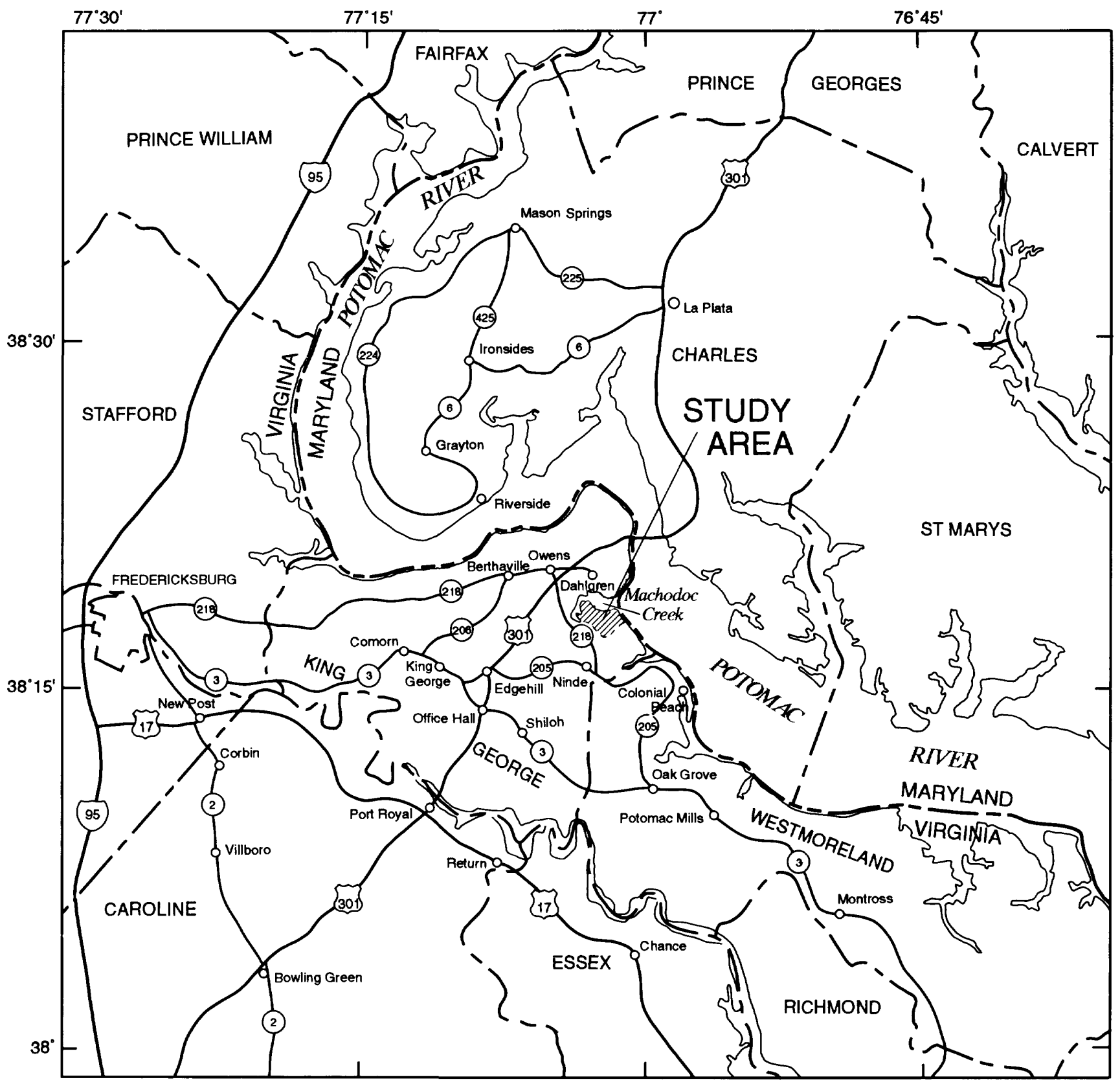

Base from U.S. Geologlcal Survey State base map, 1:500,000
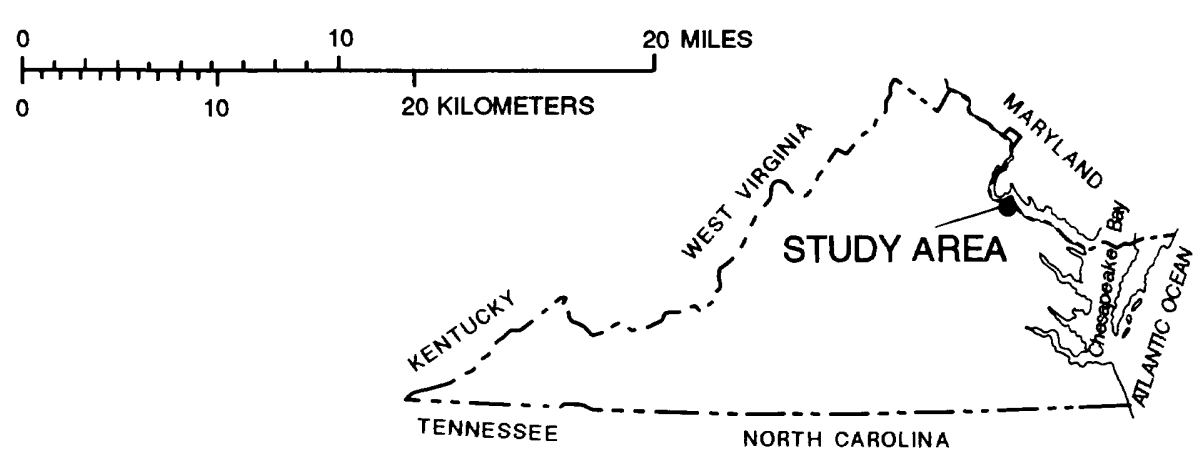

Figure 1. Location of study area at the Explosive Experimental Area, Naval Surface Warfare Center, Dahlgren Site, Dahlgren, Virginia. 
S8, S11) were drilled by hydraulic rotary method. Four wells were screened in the upper confined aquifer and four wells were screened in the Nanjemoy-Marlboro confining unit by hydraulic rotary methods. In order to avoid possible contamination of the confined aquifers from the Columbia aquifer during drilling, 10-in. insidediameter PVC casing was set and grouted into the upper confining unit before drilling continued through the aquifer below. Wells in the Columbia aquifer were constructed with 5-, 10-, 15-, or 20-ft screens, wells in the upper confined aquifer were constructed with 10-foot screens, and wells in the Nanjemoy-Marlboro confining unit were constructed with $10-\mathrm{ft}$ screens. Stainless steel centralizers were placed at the top and bottom of well screens. Details on the construction of individual wells are presented in table 1 and figure 3 .

\section{Location and Numbering System of Wells}

The 20 observation wells in the Columbia aquifer are each identified with a local number preceded by the letters "EEA-S" (EEA-S1 to S20). The eight wells in the upper confined aquifer and Nanjemoy-Marlboro confining unit are identified by a local number preceded by the letters "EEA-M" (EEA-M1 to M8). The locations of these wells on the installation are shown on plate 1 .

A unique USGS identifier was assigned to each well for the Ground-Water Site-Inventory file, a national data base maintained by the USGS. These USGS identifiers are based on the Virginia coordinate grid number of the USGS standard series 7.5-minute topographic quadrangle. Each well is numbered in accordance with the grid of its location and the chronological order in which the well was installed. For example, the USGS number $54 \mathrm{Q} 22$ corresponds to the $22 \mathrm{~d}$ well installed by the USGS in the area covered by the Dahlgren quadrangle, which has a Virginia coordinate grid number of $54 \mathrm{Q}$.

The latitudes and longitudes of observation wells were determined by a Magellan (NAV 5000 PRO) portable Global Positioning System receiver, and checked on topographic maps. Elevations of landsurface datums and measuring points were leveled in from bench marks by the USGS to an accuracy of $0.01 \mathrm{ft}$.

\section{HYDROGEOLOGIC DATA}

This section describes lithologic and geophysical logs for observation wells, mineralogic and vertical hydraulic conductivity data from laboratory analysis of 36 Shelby tube core samples, and hydrologic data from water-level measurements and aquifer tests.

\section{Lithologic and Geophysical Logs}

Sediment samples were recovered using split-spoon samplers and lithologic descriptions were logged during drilling operations at 20 observation wells. Grain size according to the Wentworth scale (Leeder, 1982) was recorded, as well as color (Munsell Color, 1975), sedimentary structures, degree of moistness, and other observations, such as the presence of organic material, shell material, indurated material, glauconite, or mica (table 2). At some intervals, no representative material was recovered with the samplers.

Geophysical logs were recorded at all drill sites (figs. 4-12). A probe was lowered to the bottom of the borehole and the geophysical response was recorded as the probe was raised. Natural gamma-radiation logs were run at all wells in the Columbia aquifer, the upper confined aquifer, and the Nanjemoy-Marlboro confining unit after the installation of casing. Natural gammaradiation, spontaneous potential, single-point resistance, 16-in. normal resistivity, and 64-in. normal resistivity logs were run at boreholes for wells screened in the upper confined aquifer and Nanjemoy-Marlboro confining unit prior to installation of the casing; natural gammaradiation and electromagnetic (EM) induction conductivity logs were run at these same boreholes after installation of the casing. Along certain intervals in the wells, the EM induction conductivity signal was affected by metal centralizers, therefore, these data are not presented.

\section{Shelby Tubes}

Thirty-six Shelby tube cores from 18 observation wells were analyzed by the U.S. Army COE, Ohio River Division Laboratory, Cincinnati, Ohio, for vertical hydraulic conductivity (table 3), and mineralogy (table 4). Vertical hydraulic conductivity was measured by the falling-head permeameter method. Mineralogy was determined by visual inspection and $\mathrm{x}$-ray diffraction. The core intervals were chosen to characterize vertical and areal variations in lithology. 


\section{Hydrologic Data}

Water levels in wells were monitored to determine the response of ground-water levels to precipitation, tidal influence, and evapotranspiration. Precipitation data, not shown in this report, were collected by the NSWCDL. Measurements of tidal fluctuations, also not shown in this report, were collected by the USGS from a tide gage installed on the north side of Upper Machodoc Creek.

\section{Ground-Water Levels}

Water levels were measured periodically at each of the 28 observation wells installed for the study (table 5). Measuring points were established for each well on the top of the casing; the elevations of these measuring points were surveyed by the USGS from bench marks to an accuracy of $0.01 \mathrm{ft}$. Water levels in wells were measured to an accuracy of $0.01 \mathrm{ft}$ by chalked steel tape. These water-level measurements were then subtracted from the elevations of the respective measuring points to obtain the elevations of the water levels.

In addition to the periodic measurements, ADR's were installed on 10 wells (EEA-M1, S1, M3, S3, M4, S4, M6, S6, M8, and S8) for the continuous monitoring of water levels. The ADR's recorded measurements every hour. Continuous (hourly) data are used to observe seasonal and short-term variations in water levels, and to allow direct comparison of the effects of tidal influences, precipitation events, and other hydrologic variables on water levels in different aquifers. Water-level data from ADR's are presented in hydrographs, showing minimum daily water levels (relative to sea level) (figs. 13-22) from March 1994 to April 1995.

\section{Slug Tests}

Slug tests, by the volume-displacement method, were conducted on all 28 observation wells to determine horizontal hydraulic conductivity. An In-Situ Hermit (1000B) data logger and pressure transducer were used to record the water levels. Prior to the introduction of the slug, the water level was monitored for 10 minutes so that any rising or falling background trend could be established and subtracted from the water-level recovery. The slug was introduced by a sand-filled PVC cylinder of known volume. The cylinder was rapidly lowered into the well, causing a temporary rise in the water level. The data logger recorded the rise and decline of the water level over time until the water level returned to equilibrium. Instantaneous water levels were recorded at intervals ranging from every 0.2 seconds at the beginning of the test to every 30 seconds at the end of the test (table 6). An additional slug test was performed on some wells by recording the rise in water level after removal of the slug from the well and the subsequent rise to equilibrium. Rising-head and falling-head data from slug tests are presented in graphs that show the depth of the water level below the measuring point over the course of the slug test (figs. 23-49).

\section{WATER-QUALITY DATA}

Field parameters and the concentrations of selected chemical constituents were measured in ground-water and surface-water samples from the NSWCDL. This water-quality data can be used to assess background geochemistry of ground water and surface water at the NSWCDL. Reporting limits for all water-quality constituents are given in table 7. Chemical analyses of surface-water and ground-water samples are listed in tables 8 and 9 , respectively.

\section{Location and Methods of Field Sampling}

Water samples were collected from the 28 observation wells, from 2 surface-water sites in Machodoc Creek, and from 1 surface-water site in Black Marsh (pl. 1) in July 1994. Ground-water samples were collected using a stainless steel submersible pump with a Teflon discharge tube. Each well was purged of at least three well volumes of water prior to sampling, unless the well went dry in which case the water level was allowed to recover prior to sampling. Surface-water samples were collected with a weighted-bottle sampler using the equal-width-increment method (Edwards and Glysson, 1988). Prior to collecting water-quality samples, all equipment was cleaned thoroughly with laboratory detergent and rinsed with tap and distilled water. The outside of the pump and Teflon tubing were rinsed with distilled water between wells, and the entire system was flushed with distilled water after each day of sampling.

Temperature, dissolved oxygen (DO), specific conductance, $\mathrm{pH}$, and alkalinity were measured in the field. Temperature and DO were measured in wells and surface water after sampling, using a YSI (model 54A) dissolved oxygen meter with cable and submersible stirrer. The DO meter was calibrated to air at least once 
per day. Specific conductance and $\mathrm{pH}$ were measured in unfiltered water samples using an Orion (model 122) specific conductance meter with glass conductivity cell and Orion (SA 250) pH meter with gel-filled pH electrode, respectively. The calibration of the specific conductance meter was checked each day using three solutions of known conductance. The $\mathrm{pH}$ meters were calibrated each day using two solutions of known $\mathrm{pH}$ values (usually pH 4 and 7). Alkalinities were determined by incremental titration of $100-\mathrm{mL}$ filtered water samples with sulfuric acid.

Water samples collected for dissolved inorganic analyses were filtered immediately after collection to minimize oxidation of any chemically reduced solutes and then placed in acid-rinsed bottles. The filtering was accomplished using a peristaltic pump and a 142-mmdiameter filter-plate assembly with $0.45-\mathrm{mm}$ pore-size filter membrane. The filter membranes were flushed with $200 \mathrm{~mL}$ of American Society of Testing and Materials Type II water before filtration of the sample. Water samples collected for major cations and metals were acidified to $\mathrm{pH} 2$ with concentrated nitric acid. All bottles containing water samples were placed in sealed plastic bags, chilled with ice, and shipped overnight to the laboratories in high-impact plastic ice chests.

Quality controls included duplicate samples, an equipment blank, and a carbon-free de-ionized water blank. Duplicate samples were collected at approximately 10 percent of the sampling sites, including one duplicate surface-water sample, to determine the reproducibility of water-quality data. Analyses of duplicate samples are presented in tables 8 and 9 with results from the other samples. A carbon-free de-ionized water blank and an equipment blank of carbon-free deionized water were passed through the pump and tubing to determine the cleanliness of equipment and samplecollection procedures.

\section{Methods of Laboratory Analysis}

All water-quality samples were sent to the USGS National Water Quality Laboratory in Arvada, Colo., for determination of inorganic constituents and organic carbon. Metals and major cations were determined using inductively coupled argon plasma. Chloride and fluoride were determined by a colorimetric method, and sulfate was determined by a turbidimetric method. Total dissolved solids were measured as residue-onevaporation at $180^{\circ} \mathrm{C}$. The analytical methods are described in Fishman and Friedman (1989) and Wershaw and others (1987). Laboratory quality-assurance procedures are described in Friedman and Erdmann (1982) and Jones (1987).

\section{DATA MANAGEMENT}

Data from field activities were recorded in bound notebooks and validated by the field personnel and a reviewer. Well-construction data and periodic waterlevel measurements were entered into the USGS GroundWater-Site-Inventory file, a storage and retrieval system that is part of the National Water Information System (NWIS). The continuous ground-water-level data were entered into the USGS Automated Data Processing System (ADAPS), which is also part of NWIS. ADAPS consists of a collection of computer programs and files designed to provide a comprehensive system of up-todate standardized water-data processing procedures. All water-quality data from the USGS National Water Quality Laboratory were reviewed and verified by laboratory and project personnel, and entered into the USGS Quality of Water Data Base, which is part of NWIS. The original field notebooks and hard copies of all well-construction, water-level, and water-quality data are stored at the USGS, Virginia District office, in Richmond, Va.

\section{REFERENCES CITED}

Bell, Clifton F., Bolles, Thomas P. and Harlow, George E. Jr., 1994, Hydrogeologic and Water-Quality Data for the Main Site, Naval Surface Warfare Center, Dahlgren Laboratory, Dahlgren, Virginia: U.S. Geological Survey Open-File Report 94-301, $81 \mathrm{p}$.

Edwards, Thomas K., and Glysson, G. Douglas, 1988, Field methods for measurement of fluvial sediment: U.S. Geological Survey Open-File Report 86-531, 118 p.

Fishman, M.J., and Friedman, L.C., 1989, Methods for the determination of inorganic substances in water and fluvial sediments (3d ed.): U. S. Geological Survey Techniques of Water-Resources Investigations, book 5, chap. A1, 545 p.

Fred C. Hart Associates, 1983, Initial Assessment Study of Naval Surface Weapons Center/Dahlgren Laboratory: Naval Energy and Environmental Support Activity, contract no. N62474-81-C-9384, 68 p. 
Friedman, L.C., and Erdmann, D.E., 1982, Quality assurance practices for the chemical and biological analyses of water and fluvial sediments: U. S. Geological Survey Techniques of Water-Resources Investigations, book 5, chap. A6, $181 \mathrm{p}$.

Halliburton NUS, 1995, Final Installation Restoration Site Management Plan for the Naval Surface Warfare Center Dahlgren Laboratory, Dahlgren, Virginia: Northern Division Naval Facilities Engineering Command, contract no. N62472-9D-D-1298, contract task order 0118, 76 p.

Jones, B.E., 1987, Quality control manual of the U. S. Geological Survey's National Water Quality Laboratory: U.S. Geological Survey Open-File Report 87-451, 17 p.

Leeder, M.R., 1982, Sedimentology: George Allen and Unwin Ltd., London, UK, 344 p.

Meng, A.A., III, and Harsh, J.F., 1988, Hydrogeologic framework of the Virginia Coastal Plain: U. S. Geological Survey Professional Paper 1404-C, 82 p.
Mixon, R.B., Berquist, C.R. Jr., Newell, W.L., and Johnson, G.H., 1989, Geologic map and generalized cross sections of the Coastal Plain and adjacent parts of the Piedmont, Virginia: U. S. Geological Survey Miscellaneous Investigations Series, Map I-2033, 2 sheets.

Munsell Color, 1975, Munsell color charts: Macbeth, a division of Kollmorgen Instruments Corporation, Baltimore, Md., $21 \mathrm{p}$.

O'Brien and Gere Engineers, Inc., 1986, Confirmation Studies at Naval Surface Weapons Center, Dahlgren, Virginia: Chesapeake Division Naval Facilities Engineering Command, contract no. P11 N62477-83-C-0113, 141 p.

Wershaw, R.L., Fishman, M.J., Grabbe, R.R., and Lowe, L.E., eds., 1987, Methods for the determination of organic substances in water and fluvial sediments: U. S. Geological Survey Techniques of Water-Resources Investigations, book 5 , chap. A3, $80 \mathrm{p}$. 


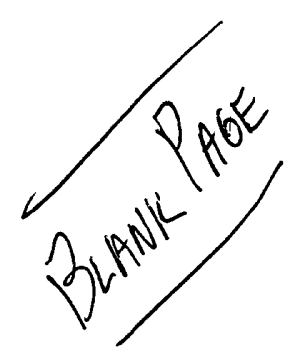


TABLES 
$\operatorname{rin} t a r+t a b i$

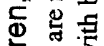

$\begin{array}{llllll}0 & 0 & 0 & 0 & 0\end{array}$

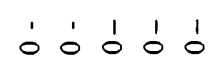

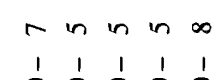

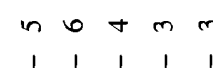

$\begin{array}{lll}7 & 7 & 0 \\ 1 & 1 & 1 \\ \vdots & 0 & 0\end{array}$

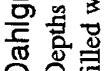

के

品

훙

范初

홍

要

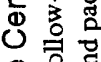

o 항

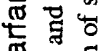

3.

\&

की

舀离

2

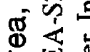

安田

을 을

通 중

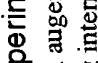

齐

is

응

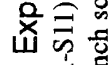

畄守

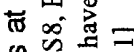

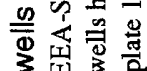

要

密尊

$\eta=m m a$ 유

$\begin{array}{rrrrr}1 & 1 & 4 & 4\end{array}$

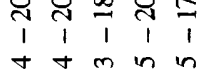

กี สิ

$\sin a n$

$+m$ in in

$\begin{array}{rrrr}1 & 1 & 1 \\ - & n & \text { in } & \infty\end{array}$

주군

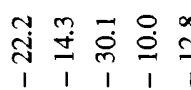

ํํำ ำ

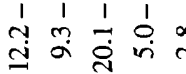

$\begin{array}{lllll}1 & 1 & 1 & 1 & 1\end{array}$

ปิ

舟官官

$\begin{array}{ccccc}1 & 1 & 1 & 1 & 1 \\ \infty & 0 & -a & a & 0\end{array}$

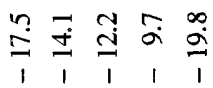

ก

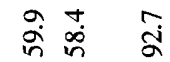

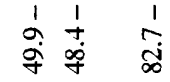

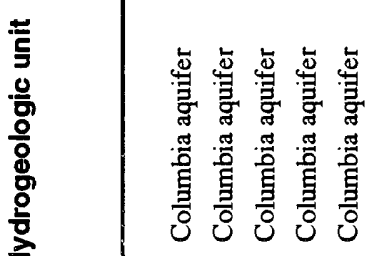

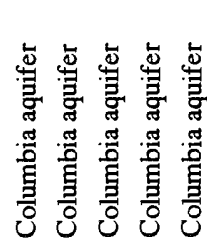

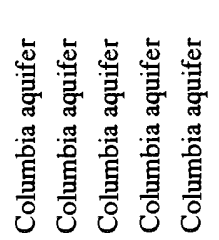

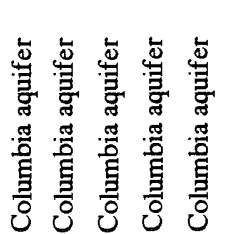

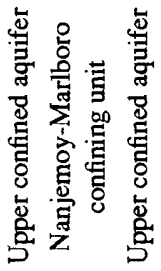

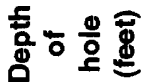

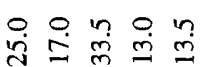

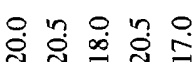

웅요융ำ

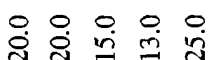

오융

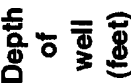

正焉

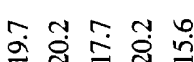

m

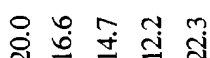

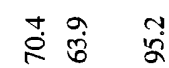

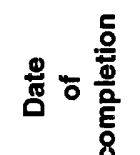

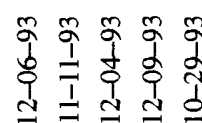

ติ

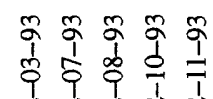

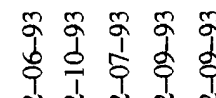

$\begin{array}{lll}a & m & a \\ \alpha & 0 & o \\ 1 & 1 & 9\end{array}$

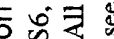

䎡市势

고웡

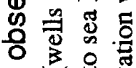

는

(1) 윤

舟

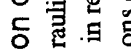

은 홀

总 总总宫

ठํํㄹ

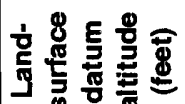

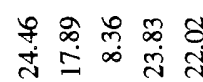

$\stackrel{1}{=} \pm \stackrel{1}{=}$

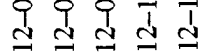

ป ป

홈

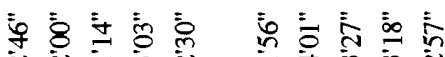

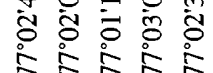

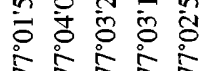

究帚

享。

$\pi$ 유 $m$

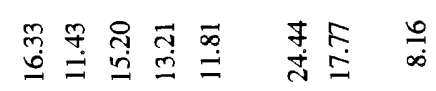

鄫

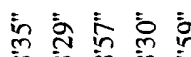

$\infty \underset{\infty}{\infty} \stackrel{\infty}{\infty} \stackrel{m}{\infty}$

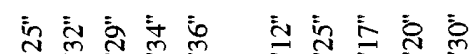

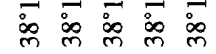

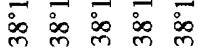

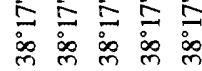

艺 三

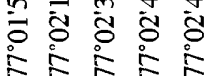

i

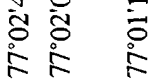

矛 $\overline{0}$

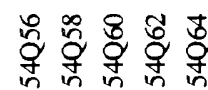

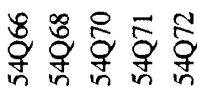

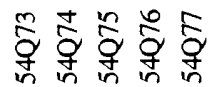

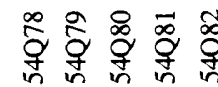

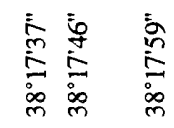

论

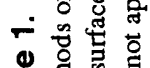

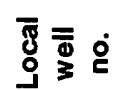

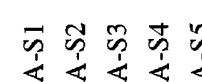

ஸ क क के

䒺离离出

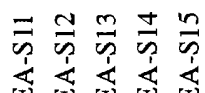

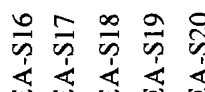

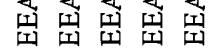

牙㓙田四田

空出出出出

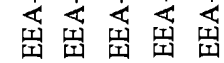

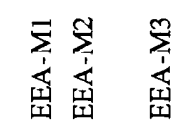




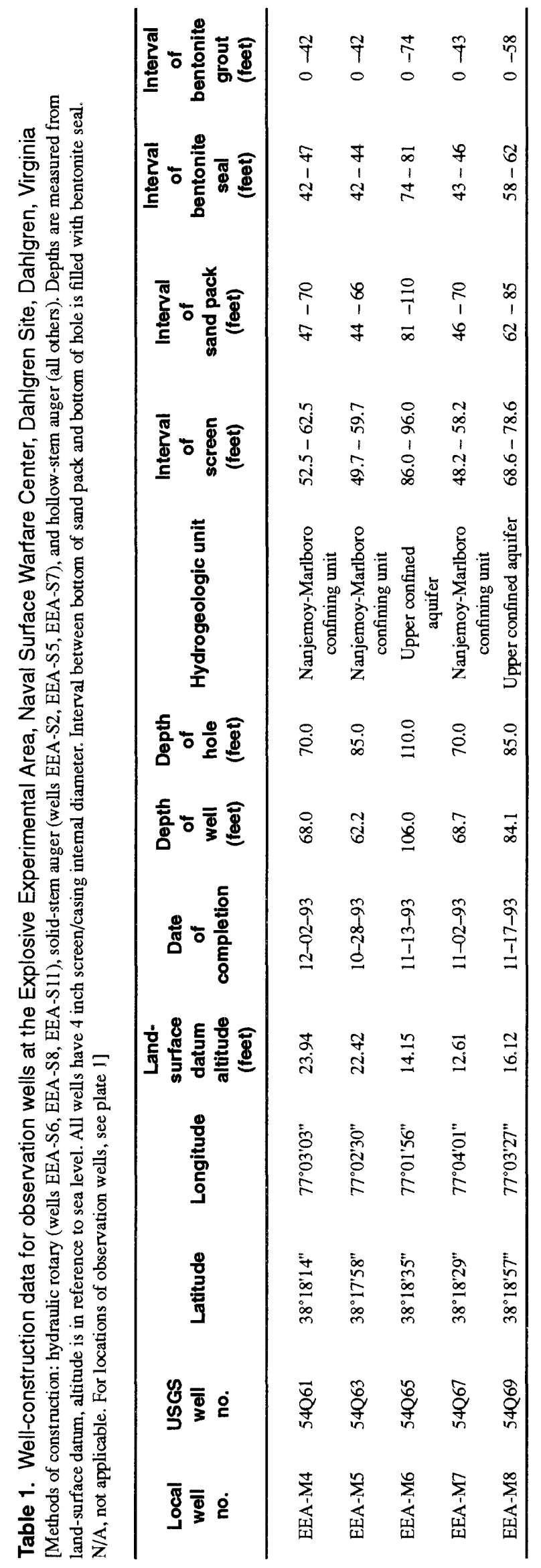


Table 2. Lithologic logs of observation wells at the Explosive Experimental Area, Naval Surface Warfare Center, Dahlgren Site, Dahlgren, Virginia

[in., inch; ft, foot; $\mathrm{cm}$, centimeter, $\mathrm{mm}$, millimeter;\%, percent: <, less than; >, greater than; depth, feet below land surface datum; for locations of observation wells, see plate 1; codes in parentheses are color codes according to Munsell Color (1975), grain size is according to Wentworth scale (Leeder, 1982)]

\section{Depth (feet) Description \\ Well EEA-S9 (54Q 71) \\ $0.0-2.0$ \\ 2.5-5.0 \\ $5.0-7.0$ \\ $10.0-12.0$ \\ 17.0 \\ Clay, (10YR 6/2) and (10YR 7/4) and (10YR 6/6), plastic, no mica, no glauconite. Mottled. \\ Clayey silt which grades downward into silty sand, very-fine-grained, (5Y 6/4), some mica, some dark minerals. Silty sand, very-fine-grained, (5Y 5/2) and (10YR 6/6), micaceous. Contains ironstone nodules. Mottled. Silty pebbly clayey sand, very-fine-grained, (5Y 6/1) to (5Y 4/1), very poorly sorted, some mica, mottled, grades downward into silty sand, very-fine-grained, (5Y 6/1) to $(5 Y 4 / 1)$, micaceous, some glauconite, with clasts of clay, (10YR 4/2). \\ Silty sand, very-fine-grained, (5GY 4/1), micaceous, glauconitic. Contains moderate numbers of bivalve fragments.}

$0.0-2.0$

2.0-5.0

5.0-7.0

9.0

$10.0-15.5$

$15.5-17.0$

$0.0-3.2$

$3.2-4.0$

4.0-6.0

6.0-8.0

$8.0-10.0$

$10.0-12.0$

$12.0-14.0$

$14.0-16.0$

$16.0-18.0$

18.0-20.0

20.0-22.0

24.5

$30.0-31.8$

31.8-32.0

34.0

\section{$0.0-2.0$}

2.0-5.0

$5.0-7.0$

\section{Well EEA-S10 (54Q 72)}

Silty clay, (10YR 6/2), no mica, no glauconite. Slightly mottled.

Silty clayey sand, very-fine- to fine-grained, (5Y 6/1), no mica, no glauconite.

Sand, very-fine- to medium-grained, (5YR 5/2) grading downward to (10YR 7/4), little mica, 3-5\% dark minerals

Driller indicates greenish sediment on augers

Silty sand, very-fine-grained, (5GY 2/1), micaceous, some glauconite. Massively bedded.

Silty sand, very-fine-grained, (5Y 4/1), micaceous, some glauconite. Contains abundant Macrocallista.

\section{Well EEA-S11 (54Q 73)}

Clayey silt, (10YR 6/2) to (10YR 4/2), micaceous, no glauconite. Contains roots and other plant material. Sand, very-fine- to fine-grained, (10YR $8 / 2$ ), well-sorted, some dark minerals.

Sand, fine- to coarse-grained, interbedded $(3-5 \mathrm{~cm})$ with sand, very-fine- to fine-grained, well-sorted. Both sediments mottled, color (5YR 5/6) and (10YR 8/2) and (10YR 6/6). Contains 30-cm diameter quartz pebble. Sand, very-fine- to fine-grained, (10YR 8/2), well-sorted, some mica, some dark minerals. Slightly mottled. Sand, very-fine-grained, (5YR 5/6) and (10YR 6/2), well-sorted, some mica some dark minerals. Mottled. Silty sand, very-fine-grained, (5Y 6/1), some mica, some dark minerals.

Clayey sandy silt, (5Y 6/1) to (5Y 4/1), micaceous, some dark minerals. Sand is very-fine-grained.

Sandy silt, (5Y 4/1), some mica, some dark minerals. Sand is very-fine-grained.

Sand, fine- to very-fine-grained, (5G 6/1) little mica, some dark minerals.

Sand, very-fine to fine-grained, (5G 6/1), some mica, some dark minerals.

Sand, very-fine-grained, (5G 6/1), some mica, some dark minerals.

Sand, very-fine-grained, (5Y 6/1), some mica, some dark minerals. Contains little plant material (leaves).

Sand, fine- to medium-grained, (5Y 6/1) to (5Y 4/1), some mica, some dark minerals.

Clay, (5Y 4/1), plastic, little mica, no glauconite. Contains black (organic?) streaks and plant material.

Clay, (5GY 6/1) to (5Y 6/1), plastic, no mica, no glauconite. Contains very chalky carbonate fragments.

\section{Well EEA-S12 (54Q 74)}

Sandy silt, (10YR 5/4) to (10YR 4/2), some mica, no glauconite. Sand is very-fine-grained. Contains modern roots and other plant material.

Sand, very-fine- to medium-grained, (10YR 6/6), little mica, some dark minerals.

Sand, as 2-5 ft interval, grades downward into pebbly sand, medium- to fine-grained, (10YR 8/2) and (10YR

7/4). Sand is subangular. Pebbles are $2-4 \mathrm{~mm}$ in diameter. Mottled. 
Table 2. Lithologic logs of observation wells at the Explosive Experimental Area, Naval Surface Warfare Center, Dahlgren Site, Dahlgren, Virginia-Continued

\section{Well EEA-S12 (54Q 74)-Continued}

7.0-10.0 Sand, as bottom of 5-7 ft interval except some coarse-grained sand. At $7 \mathrm{ft}$ hit lag of subrounded pebbles, 5-10 mm diameter.

10.0-12.0 Pebbly sand, medium- to fine-grained, (10YR 5/4), no mica, no glauconite. Pebbles are 5-10 mm in diameter and rounded.

14.0 Sand, fine-grained, (5Y 6/1), well-sorted, massively bedded, no mica, no glauconite.

15.0-20.4 Sand, as at $14 \mathrm{ft}$ except some mica.

20.4-22.0 Clay, (5GY 6/1) to (5GY 4/1), plastic, little mica, no glauconite. Contains black (organic?) streaks.

\section{Well EEA-S13 (54Q 75)}

0.0-2.0 Sandy silt, (10YR 5/4) to (10YR 4/2), no mica, no glauconite. Sand is very-fine-grained. Contains modern wood and roots.

3.0-5.0 Sandy silt, (10YR 5/4) to (10YR 4/2), no mica, no glauconite. Sand is very-fine-grained. Contains modern wood and roots.

5.0-10.0 Sand, fine-grained, (10YR 8/2) and (10YR 7/4), well-sorted, some dark minerals. At $6 \mathrm{ft}$ hit thin pebble lag.

10.0-12.0 Sand, as 5-10 ft interval except has thin $(<1 \mathrm{~mm})$ bands of iron staining, (10YR 6/6).

15.0-17.0 Sand, medium-grained, (10YR 8/2) to (10YR 7/4), no mica, some dark minerals. Contains pebbles, 5-10 mm diameter, at $16.5-17.0 \mathrm{ft}$.

20.0-22.0 Sand, fine- to very-fine-grained, some medium-grained, (10YR 7/4) and (5YR 5/6), some dark minerals. Contains $2-3 \mathrm{~cm}$ bands of iron staining.

25.0-27.0 Not recovered.

25.0-30.0 Sand, fine-grained, (5Y 6/1), well-sorted.

$30.0-32.0$

37.0

Sand, very-fine-grained, (5Y 6/1), well-sorted, no mica, some dark minerals.

Clay, (5GY 4/1), plastic, some mica, no glauconite.

\section{Well EEA-S14 (54Q 76)}

0.0-2.0 Sand, very-fine-grained, (10YR 7/4) to (10YR 5/4), no mica, some dark minerals. Contains moderns roots and leaves.

2.0-5.0 Pebbles at 2.5-3.0 ft High TIP reading and root-like odor at $5.0 \mathrm{ft}$.

5.0-6.8

6.8-7.0 Pebbly sand, fine- to medium-grained, (10YR 8/6) to (10YR 6/6), no mica, no glauconite. Pebbles are 4-12 mm in diameter.

10.0-12.0 Sand, fine- to medium-grained. Very mottled. $10.5 \mathrm{ft}$.

15.0-15.5 Silty sand, very-fine-grained, (10YR 6/6) and (10YR 5/4), no mica, some dark minerals.

15.5-17.0 Silty clayey sand, very-fine-grained, (10YR 5/4) grading downward into (5GY 4/1), some mica.

21.0 Silty sand, very-fine-grained, (5GY 4/1), micaceous, some glauconite.

\section{Well EEA-S15 (54Q 77)}

0.0-2.0 Silty sand, very-fine-grained, (10YR 5/4), some mica, no glauconite. Contains abundant modern root and leaves.

4.0

5.0-7.0

$7.0-10.0$

$10.0-12.0$

$15.0-17.0$
Thin pebble lag.

Sand, very-fine-grained, (N9), well-sorted, massively bedded, no mica, no glauconite.

Sand, fine-grained, (10YR 8/6), well-sorted, no mica, some dark minerals. Dry.

Sand, very-fine- to fine-grained, some medium-grained, (10YR 8/2) and (10YR 7/4) and (10YR 6/6), well-

sorted, no mica, some dark minerals. Mottled. Dry.

Sand, very-fine-grained, (10YR 8/2), well-sorted, micaceous, some dark minerals. 
Table 2. Lithologic logs of observation wells at the Explosive Experimental Area, Naval Surface Warfare Center, Dahlgren Site, Dahlgren, Virginia-Continued

\begin{tabular}{ll}
\hline Depth (feet) & \multicolumn{1}{c}{ Description } \\
\hline Well EEA-S15 (54Q 77)-Continued \\
20.0-21.7 & $\begin{array}{l}\text { Sand, medium- to very-fine-grained, some coarse-grained, (5YR 6/6) and (5Y 7/2), little mica, some dark } \\
\text { minerals, grades downward into silty sand, very-fine-grained. Mottled. }\end{array}$ \\
$21.7-22.0$ & $\begin{array}{l}\text { Clayey silt, (5GY 4/1), micaceous, some glauconite, very-fine-grained. Contains clasts of clay, (10YR 4/2). } \\
\text { Clayey sandy silt, micaceous, some glauconite. Sand is very-fine-grained. Contains poorly preserved wood } \\
\text { fragments. }\end{array}$
\end{tabular}

Well EEA-S16 (54Q 78)

0.0-2.0 Clayey sandy silt, (10YR 6/2), little mica, no glauconite. Sand is very-fine-grained. Contains modern roots, other plant material. Slightly mottled.

2.0-5.0 Sand, very-fine-grained, (10YR 8/2) to (10YR 7/4), no mica, no glauconite. Mottled.

5.0-7.0 Sand, very-fine grained grading downward to fine-grained, (10YR 7/4) to (10YR 6/2), some mica, no glauconite. Mottled.

7.0-9.0 Silty sand, fine- to very-fine-grained, (10YR 7/4) to (10YR 6/2).

9.0-11.3 Silty sand, fine- to very-fine-grained, (10YR 8/2) and (10YR 6/6), some mica, no glauconite. Mottled. Sediments wet at approximately $10 \mathrm{ft}$.

11.3-12.0 Sand, medium- to fine-grained, (N7) and (10YR 6/6), no mica. Mottled.

13.5-15.0 Silty clayey sand, very-fine-grained, (5GY 4/1), very micaceous, no glauconite. Contains poorly preserved organic material. Color change occurs at $13.5 \mathrm{ft}$.

15.0-20.0 Silty sand, very-fine-grained, (5Y 4/1), very micaceous, some dark minerals. Bioturbated appearance.

20.0-22.0 Silty sand, very-fine-grained, (5GY 4/1), micaceous, glauconitic. Bioturbated. Contains abundant Macrocallista shell and shell fragments. Contains clasts of silty clay, (10YR $4 / 2$ ), very micaceous.

\section{Well EEA-S17 (54Q 79)}

0.0-2.0 Silty pebbly sand, very-fine-grained, (10YR 5/4) to (10YR 4/2), very poorly sorted, some mica, no glauconite. Pebbles are $2-5 \mathrm{~mm}$ in diameter. Contains modern roots.

2.0-5.3 Clayey sandy silt, (10YR 6/6) to (10YR 5/4). Sand is very-fine-grained. Contains some plant material.

5.3-7.0 Clay, (10YR 7/2) and (5YR 5/6), plastic, no mica, no glauconite. Contains ironstone nodules (2-5 mm diameter). Mottled. Driller reports "gravel sound" at $7 \mathrm{ft}$.

10.0-12.0 Silty pebbly sand, very-fine-grained, (10YR 4/2), little mica, grades downward into sand, very-fine-grained, (10YR 7/4) and (5YR 5/6), well-sorted, some mica, some dark minerals. Wet.

15.0-17.0 Silty clay, (5Y 4/1), micaceous, no glauconite. Massively bedded.

22.0 Clay, (5Y 4/1), plastic, micaceous, some dark minerals. Contains rare white chalky grains $(<1 \mathrm{~mm}$ diameter $)$.

\section{Well EEA-S18 (54Q 80)}

0.0-2.0 Sandy clayey silt, (10YR 6/2) to (10YR 4/2), no mica, no glauconite. Sand is very-fine-grained. Contains modern roots and other plant material. Slightly mottled.

2.0-3.0 Silty clay, (10YR 6/2) and (10YR 6/6), plastic, no mica, no glauconite. Very mottled.

$5.0-7.0$

$10.0-12.0$

17.0 Clay, (10YR 6/2) and (10YR 6/6), plastic, no mica, no glauconite. Very mottled. Wet at $9 \mathrm{ft}$.

Silty clay, micaceous, some glauconite, grades downward into sandy clayey silt, (5GY 4/1), micaceous, some glauconite. Sand is very-fine-grained. Lower unit contains streaks of clay, (5YR 3/2), micaceous, no glauconite. Silty sand, very-fine-grained, (5GY 4/1), micaceous, glauconitic. Contains abundant Macrocallista, rare Turritella. 
Table 2. Lithologic logs of observation wells at the Explosive Experimental Area, Naval Surface Warfare Center, Dahlgren Site, Dahlgren, Virginia-Continued

\begin{tabular}{ll}
\hline Depth (feet) Description \\
\hline
\end{tabular}

Well EEA-S19 (54Q 81)

0.0-2.0 Silty sand, very-fine-grained, (10YR 6/2) to (10YR 4/2), no mica, no glauconite. Contains modern roots. Probably fill material.

3.0 Clayey sandy silt, (5Y 4/1), little mica, no glauconite. Sand is very-fine-grained.

5.0-7.0 Silty sand, fine- to very-fine-grained, (10YR 4/2), some mica, no glauconite. Contains wood fragments. Color changes to $(5 Y$ 6/1) at $6.5 \mathrm{ft}$. Possibly fill material.

10.0-10.5 Silty sand, very-fine-grained, (5Y 4/1), micaceous, glauconitic. Massively bedded. Slightly mottled.

10.5-12.0 Silty sand, very-fine-grained, (5Y 4/1) and (5GY 4/1), micaceous, glauconitic. Bioturbated. Contains abundant Macrocallista.

$15.0 \quad$ Silty sand, as 10.5-12 ft interval. Contains Macrocallista, Venericardia, one scaphopod shell (Dentalium?) noted.

\section{Well EEA-S20 (54Q 82)}

0.0-2.0 Clayey silt, (10YR 6/2) to (10YR 4/2), no mica, no glauconite.

3.0-7.0 Clay, (10YR 7/4) and (10YR 6/6), plastic, little mica, no glauconite. Mottled.

7.0 Clayey pebbly sand, fine- to medium-grained, (10YR 6/2) to (10YR 4/2), poorly sorted. Pebbles are $10-25 \mathrm{~mm}$ in diameter, subrounded.

10.0-12.0 Clay, (10YR 6/2) and (5YR 5/6), plastic, little mica, no glauconite. Contains small (1-5 mm) nodules of ironstone. Mottled.

15.0-17.0 Clay, as 10-12 ft interval.

17.0

20.0-22.0

$25.0-27.0$

Sand, very-fine-grained, (5Y 6/1) to (5Y 4/1), well-sorted, some mica, no glauconite.

Sand, very-fine-grained, (5Y 6/1) to (5Y 4/1), well-sorted, micaceous, some dark minerals.

Clayey silt grading downward into clay, (5Y 6/1) to (5Y 4/1), micaceous, no glauconite.

\section{Well EEA·M1 (54Q 55)}

0.0-2.0 Clayey sandy silt, olive-gray (5Y 4/1), little mica, no glauconite. Sand is very-fine-grained. Contains plant material. Slightly mottled. Dry.

2.0-4.0 Clay which coarsens downward into silty clay, very-pale-orange (10YR 8/2) and pale-yellowish-brown paleyellowish-brown (10YR 6/2), no mica, no glauconite. Top 6 in. is organic rich. Mottled. Dry.

4.0-4.5 Silty clay, very-pale-orange (10YR 8/2) and brownish-gray (5YR 4/1), no mica, no glauconite. Slightly mottled. Pebble lag at $4.5 \mathrm{ft}$.

4.5-6.0 Pebbly sand, very-pale-orange (10YR 8/2) to pale-yellowish-brown (10YR 6/2), very-fine- to fine-grained, wellsorted, some mica, no glauconite. Pebbles are $1-10 \mathrm{~mm}$ in diameter, angular.

6.0-8.0 Sand, light-gray (N6) and grayish-orange (10YR 7/4), very-fine- to medium-grained, about $1 \%$ dark minerals, no mica, no glauconite. Slightly mottled.

8.0-10.0 Pebbly sand, very-pale-orange (10YR 8/2) to pale-yellowish-brown (10YR 6/2), medium- to coarse-grained, no mica, no glauconite. Pebbles are 1-5 mm in diameter. Wet.

13.0

$14.0-16.0$

$18.0-19.7$

$19.7-20.0$

$22.0-24.0$

28.5

$30.0-40.0$

Sand, dark-greenish-gray (5GY 4/1), very-fine-grained, well-sorted, no mica, no glauconite.

$40.0-42.0$

$42.0-50.0$ Silty sand, olive-gray (5Y 4/1), very-fine-grained, no mica, no glauconite. Contains dark (organic?) stains and rare clay flasers.

As 14-16 ft interval except mottled; color dark-greenish-gray (5GY 4/1) and medium-bluish-gray (5B 5/1). Pebbly sand, brownish-gray (5YR 4/1), very-fine-grained. Pebbles are 1-5 mm in diameter, subangular. Silty clay, dark-greenish-gray (5GY 4/1), micaceous, no glauconite. Driller notes "good clay" at $23.5 \mathrm{ft}$. Clay, dark-greenish-gray (5G 4/1), plastic, some mica, no glauconite. Contains rare plant material. Clay, as at $28.5 \mathrm{ft}$. Abundant wood fragments/ plant material. Clay, dark-greenish-gray (5G 4/1), plastic, micaceous, no glauconite. Rare plant material. Clay, as 40-42 ft interval, some sand, very-fine- to fine-grained, some pebbles $(1-5 \mathrm{~mm})$. Contains abundant plant material. Clay speckled. Driller notes "sand" at $44-48 \mathrm{ft}$; "gravel" at $48 \mathrm{ft}$; "out of gravel" at $49.5 \mathrm{ft}$. 
Table 2. Lithologic logs of observation wells at the Explosive Experimental Area, Naval Surface Warfare Center, Dahlgren Site, Dahlgren, Virginia-Continued

\begin{tabular}{ll}
\hline Depth (feet) & Description \\
\hline
\end{tabular}

\section{Well EEA-M1 (54Q 55)-Continued}

50.0-52.0 Sand, dark-greenish-gray (5GY 4/1), very-fine-to fine-grained, well-sorted, micaceous, some glauconite.

50.0-60.0 Clay, as 40-50 ft interval, and sand, very-fine- to fine-grained, micaceous, increasingly glauconitic. Driller notes "interbedded sand and clay."

60.0-62.0 Sand, medium- to coarse-grained, pebbles, and silty clay, interbedded, olive-gray (5Y 4/1) to dark-greenish-gray (5GY 4/1), micaceous, some glauconite in sand. Contains wood fragments.

60.0-65.0 Sand, medium- to coarse-grained. Contains wood fragments. Driller notes "much harder material” at $64 \mathrm{ft}$.

65.0-70.0 Sand, coarse- to medium-grained, and pebbles, and clay, very glauconitic.

70.0-72.0 Silty sandy clay, olive-gray (5Y 4/1), very micaceous, very glauconitic. Sand is very-fine-grained. Contains moderate numbers of bivalves (Venericardia, Macrocallista).

77.0 Silty sandy clay, greenish-black (5GY 2/1) to dark-greenish-gray (5GY 4/1), micaceous, very glauconitic. Sand is very-fine-grained. Contains bivalve fragments.

\section{Well EEA-M2 (54Q 57)}

0 -2.0 Sand is very-fine- to fine-grained. Contains rare quartz pebbles (1-5 mm), and modern rootlets. Not mottled.

2.0-4.5 Pebbly sand, dark-yellowish-orange (10YR 6/6) to moderate-yellowish-brown (10YR 5/4), very-fine- to finegrained, some mica. Slightly mottled.

4.5-5.5 Sand, grayish-orange (10YR 7/4), fine-grained, well-sorted, little mica. Contains $8 \mathrm{~cm}$ quartz pebble at $5.5 \mathrm{ft}$.

5.5-6.0 Pebbly sand, dark-yellowish-orange (10YR 6/6) to moderate-yellowish-brown (10YR 5/4), very-fine- to finegrained, poorly-sorted. Pebbles are subangular to subrounded. Mottled.

6.0-8.0 Pebbly sand, dark-yellowish-orange (10YR 6/6) to moderate-yellowish-brown (10YR 5/4), very-fine- to finegrained, poorly-sorted, some mica. Pebbles are subrounded. Contains roots and woody material. Grades downward into sand, grayish-orange (10YR 7/4) to dark-yellowish-orange (10YR 6/6), fine-grained, well-sorted, no mica, about $1 \%$ dark minerals.

9.0 Pebbly sand, very-pale-orange (10YR 8/2) to pale-yellowish-orange (10YR 8/6), fine-grained, well-sorted, about $1 \%$ dark minerals. Slightly mottled. Dry.

10.0-12.0 Pebbly sand, very-pale-orange (10YR 8/2) and dark-yellowish-orange (10YR 6/6) and light-brown (5YR 5/6), fine- to coarse-grained, pebbles are 1-5 mm in diameter. Mottled.

12.0-14.0 Pebbly sand, light-brown (5YR 5/6) and (10YR 6/2) and very-pale-orange (10YR 8/2), fine- to very-finegrained. Pebbles are 1-5 mm in diameter. Mottled. Damp. Contains pale-yellowish-brown (10YR 6/2) clay bed 13.1-13.7 ft. Damp.

14.0-16.0 Silty clayey sand, dark-greenish-gray (5GY 4/1), very-fine-grained, no mica, no glauconite.

$16.0-18.0$

19.0 Silty clay, dark-greenish-gray (5GY 4/1), some mica, no glauconite.

20.0-30.0 Silty clay, dark-greenish-gray (5GY 4/1), some mica.

Clay, as previous description; also in cuttings: clay, dark-yellowish-brown (10YR 4/2), plastic.

30.0-32.0 Clay, medium-bluish-gray (5B 5/1) to dark greenish-gray (5G 4/1), plastic, no mica, no glauconite. Contains sparse shell material, organic material. Slightly mottled.

30.0-40.0 Clay, as 30-32 ft interval until about $36 \mathrm{ft}$, where driller reports chatter. Driller says "only a few inches of gravel." Orange-stained pebbles and woody material in cuttings

40.0-42.0 Silty sand, dark greenish-gray (5G 4/1), very-fine-grained, micaceous, glauconitic. Wavy bedding. Contains sparse shell material and organic material.

40.0-45.0 Silty sand, greenish-black (5GY 2/1), very-fine-grained, micaceous, glauconitic.

45.0-50.0 Sandy silt, dark-greenish-gray (5GY 4/1), very glauconitic. Sand is very-fine-grained. Contains shell fragments. Driller reports "softer to cut" at about $48 \mathrm{ft}$.

50.0-52.0 Clayey sand, greenish-black (5GY 2/1), very-fine- to fine-grained, micaceous, glauconitic. Bioturbated. Contains moderate numbers of shells (Macrocallista).

71.5 Sandy clay, dark-greenish-gray (5GY 4/1), micaceous, glauconitic. Sand is very-fine-grained. 
Table 2. Lithologic logs of observation wells at the Explosive Experimental Area, Naval Surface Warfare Center, Dahlgren Site, Dahlgren, Virginia-Continued

\begin{tabular}{ll}
\hline Depth (feet) & Description \\
\hline
\end{tabular}

\section{Well EEA-M3 (54Q 59)}

0.0-2.0 Sandy silt, dark-yellowish-orange (10YR 6/6), very-fine-grained, no mica. Contains rare subangular pebbles and modern plant material.

2.0-4.0 Silty sand, grayish-orange (10YR 7/4) to dark-yellowish-orange (10YR 6/6), very-fine-grained, some mica. Contains subrounded pebble lag at $4.0 \mathrm{ft}$. Mottled.

4.0-4.4 Silty sand, dark-yellowish-orange (10YR 6/6), very-fine-grained, no mica. Contains pebble lag at $4.4 \mathrm{ft}$.

4.4-6.0 Silty sandy clay, pale-yellowish-brown (10YR 6/2) and dark-yellowish-orange (10YR 6/6), some mica. Sand is very-fine-grained. Contains roots and other plant material. Mottled.

6.0-8.0 Clayey sandy silt, pale-yellowish-brown (10YR 6/2) and dark-yellowish-orange (10YR 6/6), little mica, $<1 \%$ dark minerals. Sand is very-fine-grained. Contains roots and other plant material. Mottled. Moist at $7 \mathrm{ft}$.

$10.0 \quad$ Pebbly sand, grayish-orange (10YR 7/4), medium-grained

10.0-12.0 Pebbly sand, pinkish gray (5YR 8/1) to grayish-orange (10YR 7/4), medium- to coarse-grained, about $1 \%$ dark minerals. Pebbles are up to $10 \mathrm{~mm}$ in diameter, subrounded to angular.

12.0-14.0 Pebbly sand, light-gray (N6), medium- to coarse-grained, poorly-sorted, about $1 \%$ dark minerals. Pebbles are up to $10 \mathrm{~mm}$ in diameter, subrounded.

14.0-16.0 Pebbly sand, light-gray (N6), medium- to coarse-grained. Pebbles are up to 20 mm in diameter, subrounded to subangular.

16.0-18.0 Pebbly sand, moderate-yellowish-brown (10YR 5/4) to dark-yellowish-orange (10YR 6/6), medium- to coarsegrained. Pebbles are up to $20 \mathrm{~mm}$ in diameter, subrounded. Contains some silt, very little clay.

18.0-20.0 Sandy pebbles, dark-yellowish-orange (10YR 6/6) to moderate-yellowish-brown (10YR 5/4), up to $30 \mathrm{~mm}$ in diameter, subrounded to subangular. Sand is medium- to coarse-grained.

25.0-27.0 Pebbly sand, pale-yellowish-brown (10YR 6/2), medium- to coarse-grained. Pebbles are up to $5 \mathrm{~mm}$ in diameter, subangular to subrounded.

30.0-32.0 Contact at about $30 \mathrm{ft}$. Silty sand, olive-gray (5Y 4/1), very-fine-grained, well sorted, some mica, no glauconite. Massively bedded.

37.0

$40.0-42.0$

Clay, dark-greenish-gray (5GY 4/1), plastic, very little mica, no glauconite. Contains large wood fragments.

$40.0-50.0$ clams (Mercenaria?).

50.0-52.0 Clay, dark-greenish-gray (5GY 4/1), plastic, no mica, no glauconite. Massively bedded. Contains sparse organic material.

60.0-62.0 Clay, olive-gray (5Y 4/1), plastic, little mica. Massively bedded. Contains moderate amounts of organic material. $60.0-70.0$

$70.0-72.0$

81.0

$80.0-90.0$ as $60-62 \mathrm{ft}$ interval

Sand, dark-greenish-gray (5GY 4/1), medium- to fine-grained, and sandy clayey silt, dark-greenish-gray (5GY $4 / 1)$, thinly interbedded (10-30 mm), no mica, glauconitic. Coarsens downward. Sand occurs as lenses in clayey silt and is very-fine- to fine-grained. Driller notes "sand starts at about $70.5 \mathrm{ft}$."

90.0-92.0 Sand, (5GY 2/1), fine-grained, some mica, very glauconitic.

90.0-110.0 Driller notes "gravel" at $94 \mathrm{ft}$. Driller notes 'good clay" at $98.5 \mathrm{ft}$; "sand and clay" at $102 \mathrm{ft}$; "clay" at $106 \mathrm{ft}$. 112.0 Silty sandy clay, dark-greenish-gray (5GY 4/1) to greenish-black (5GY 2/1), micaceous, very glauconitic. Most sand is glauconite and is very-fine- to fine-grained. Bioturbated.

\section{Well EEA-M4 (54Q 61)}

0-2.0 Sandy clayey silt, pale-yellowish-brown (10YR 6/2), little mica. Sand is very-fine- to fine-grained. Contains modern roots. Slightly mottled.

2.0-4.0 Silty sand, pale-yellowish-brown (10YR 6/2), very-fine-grained, grading downwards into sand, pale-yellowishbrown (10YR 6/2), fine- to medium-grained, well-sorted, about $1 \%$ dark minerals. Slightly mottled.

4.0-4.5 Sand, pale-yellowish-brown (10YR 6/2), medium-grained, well-sorted. 
Table 2. Lithologic logs of observation wells at the Explosive Experimental Area, Naval Surface Warfare Center, Dahlgren Site, Dahlgren, Virginia-Continued

\begin{tabular}{ll}
\hline Depth (feet) & Description \\
\hline
\end{tabular}

\section{Well EEA-M4 (54Q 61)-Continued}

4.5-5.1 Clay, dark gray (N3), plastic. Contains roots, plant material.

5.1-5.5 Pebbly sand, olive-gray (5Y 4/1), coarse-grained. Pebbles are subrounded.

5.5-6.0 Sand, light-brown (5YR 5/6) to dark-yellowish-orange (10YR 6/6), medium-grained, poorly sorted, some mica. Mottled.

6.0-8.0 Pebbly sand, pale-yellowish-brown (10YR 6/2) and dark-yellowish-orange (10YR 6/6) and grayish-green (10GY $5 / 2$ ), medium-grained. Mottled. Grades downward into sand, greenish-black (5GY 2/1), fine- to very-finegrained, micaceous, some glauconite. Contains ghost fossils.

8.0-10.0 Silty sand, dark-greenish-gray (5GY 4/1) to olive-gray (5Y 4/1), very-fine-grained, micaceous, some glauconite. Contains some lenses of sand, very-fine-grained. Bioturbated. Dry.

10.0-12.0 Silty sand, greenish-black (5GY 2/1), fine- to very-fine-grained, some silt, micaceous, little glauconite. Massively bedded. Contains moderate numbers of bivalve fragments (2-4 mm).

12.0-14.0 Silty sand, very-fine-grained, micaceous, some glauconite (very-fine-grained). Massively bedded. Contains abundant bivalve fragments and whole shells (Macrocallista).

14.0-16.0 Silty sand, dark-greenish-gray (5GY 4/1) to greenish-black (5GY 2/1), very-fine-grained, micaceous, some glauconite (very-fine-grained). Massively bedded. Contains abundant bivalve shells (Macrocallista).

18.0-20.0 Silty sand, olive-gray (5Y 4/1) to dark-greenish-gray (5GY 4/1), very-fine-grained, micaceous. little glauconite (very-fine-grained). Contains moderate to abundant bivalve shells (Macrocallista).

21.7 Silty sand, dark-greenish-gray (5GY 4/1) to greenish-black (5GY 2/1), very-fine-grained, micaceous, little glauconite (very-fine-grained). Contains abundant bivalve shells (Macrocallista, Turritella).

20.0-30.0 As previous description, more glauconite.

30.0-32.0 Silty sand, dark-greenish-gray (5GY 4/1) to greenish-black (5GY 2/1) very-fine- to fine-grained, micaceous, some glauconite (very-fine-grained). Contains abundant large Venericardia, Macrocallista (up to $2 \mathrm{~cm}$ ).

40.0-42.0 Silty sand, greenish-black (5GY 2/1), very-fine- to fine-grained, micaceous, very glauconitic. Appears bioturbated. Contains rare bivalve fragments and clay-filled burrows.

40.0-50.0 No apparent change. Driller notes "harder" at $44 \mathrm{ft}$. Switched to rock bit; out of hard beds at $44.4 \mathrm{ft}$. Carbonatecemented Nanjemoy in cuttings.

50.0-52.0 Silty sand, dark-greenish-gray (5GY 4/1) to greenish-black (5GY 2/1), fine- to very-fine-grained, micaceous, glauconitic (fine-grained). Massively bedded. Contains rare (Venericardia). Driller notes 'sandier' at $58.8 \mathrm{ft}$.

60.0-62.0 Sand and silty clay, interbedded, dark-greenish-gray (5GY 4/1) to greenish-black (5GY 2/1), micaceous, glauconitic. Bioturbated. Sand is very-fine-grained and occurs in lenses and thin beds. Contains rare bivalves.

70.0-72.0 Clay and sand, interbedded, dark-greenish-gray (5GY 4/1) to greenish-black (5GY 2/1), micaceous. Sand is very-fine-grained and glauconitic. Bioturbated. Contains sand-filled burrows and rare Venericardia.

\section{Well EEA-M5 (54Q 63)}

$0-2.0$

2.0-3.0

$3.0-4.0$

4.0-5.0

$5.0-6.0$

$6.0-8.0$

8.0-9.0

$9.0-10.0$

$10.0-11.0$

$11.0-12.0$

$12.0-13.0$

13.0-14.0

$14.0-16.0$
Silty sand, light-olive-gray ( 5 Y $5 / 2$ ), fine- to very-fine-grained. Contains rootlets.

Silty sand, light-olive-gray (5Y 5/2), fine-grained.

Sandy silt, moderate-brown (5YR 4/1). Contains subrounded to well-rounded white quartz pebbles (2-5 mm).

Silty sand, pale-yellowish-brown (10YR 6/2), fine- to medium-grained. Contains $40 \mathrm{~mm}$ pebble lag at $5 \mathrm{ft}$.

Sand, pale-yellowish-brown (10YR 6/2), medium-grained, subangular to subrounded.

Sand, pale-yellowish-brown (10YR 6/2). medium-grained, subangular to subrounded, well-sorted.

Pebbly sand, pale-yellowish-brown (10YR 6/2), medium-grained, subangular to subrounded, well-sorted.

Pebbles are 10-15 $\mathrm{mm}$ in diameter, subangular.

Pebbly sand, yellowish-gray (5Y 7/2), medium-grained. Pebbles are angular to subangular.

Sand, yellowish-gray (5Y 7/2) to pale-yellowish-brown (10YR 6/2), medium-grained.

Silty sand, dark-greenish-gray ( $5 \mathrm{GY} 4 / 1$ to $5 \mathrm{G} 4 / 1$ ), fine- to very-fine-grained. Large rounded quartz pebble $(50 \mathrm{~mm})$ at $11 \mathrm{ft}$.

Silty sand, dark-greenish-gray (5G 4/1), fine- to very-fine-grained.

Silt, grayish-olive-green ( $5 \mathrm{GY} 3 / 2)$, some mica.

same as $13-14 \mathrm{ft}$. 
Table 2. Lithologic logs of observation wells at the Explosive Experimental Area, Naval Surface Warfare Center, Dahlgren Site, Dahlgren, Virginia-Continued

Depth (feet) Description

\section{Well EEA-M5 (54Q 63)-Continued}

18.5

20.0-22.0

$25.0-27.0$

$30.0-32.0$

$35.0-37.0$

$40.0-42.0$

$45.0-47.0$

$50.0-52.0$

$55.0-57.0$

$60.0-62.0$

$65.0-67.0$

$70.0-72.0$

$75.0-77.0$

$80.0-82.0$

$85.0-87.0$

2.5-3.7

$3.7-4.0$

$4.0-6.0$

$6.0-8.0$

8.0-10.8

$10.8-12.0$

$12.0-14.0$

$14.0-16.0$

Sand, greenish-black (5GY 2/1), fine- to very-fine-grained, micaceous, very glauconitic.

Sand, dark-greenish-gray (5GY 4/1), very-fine- to fine-grained, micaceous, glauconitic. Contains shell fragments. Contains quartz pebble $(75 \mathrm{~mm})$ at $21.5 \mathrm{ft}$ (from above?).

Silty sand, dark-greenish-gray (5GY 4/1) to greenish-black (5GY 2/1), fine-grained, micaceous, glauconitic. Contains abundant bivalve fragments and whole shells (Macrocallista, Cubitostrea), some shells articulated.

Silty sand, dark-greenish-gray (5GY 4/1), fine-grained, micaceous, glauconitic. Contains bivalve fragments and whole shells (Macrocallista, Cubitostrea). Massively bedded.

Silty sand, dark-greenish-gray (5GY 4/1), fine-grained, micaceous, glauconitic. Contains bivalve fragments and whole shells (Macrocallista, Cubitostrea). Massively bedded.

Silty sand, dark-greenish-gray (5GY 4/1) to greenish-black (5GY 2/1), medium- to fine-grained, micaceous, very glauconitic. Contains bivalves (Macrocallista, Venericardia, Cubitostrea). Massively bedded.

Silty sand, greenish-gray (5GY4/1) to greenish-black (5GY 2/1) very-fine- to medium-grained, micaceous, very glauconitic. Massively bedded. Sparsely fossiliferous (Macrocallista, Venericardia).

Silty sand, greenish-black (5GY 2/1), fine-grained, micaceous, very glauconitic. Massively bedded. Sparsely fossiliferous (Venericardia), shells chalky. Contains 3" diameter carbonate concretion at $51.7 \mathrm{ft}$.

Silty sand, greenish-black (5GY 2/1), fine- to very-fine-grained, micaceous, very glauconitic. Bioturbated. Moderately fossiliferous (Venericardia, Macrocallista).

Silty sand. dark-greenish-gray (5GY4/1), fine- to very-fine-grained. micaceous, glauconitic. Bioturbated. Sparsely fossiliferous (Venericardia).

Clayey silty sand, dark-greenish-gray (5GY 4/1) to greenish-black (5GY 2/1), fine-grained, micaceous, glauconitic. Bioturbated. Contains plant material. Fossiliferous (Venericardia, Cubitostrea).

Sandy silty clay, dark-greenish-gray (5GY4/1), micaceous, glauconitic. Sand is very-fine-grained. Sparsely fossiliferous. Contains plant stem.

Silty sandy clay, dark-greenish-gray (5GY 4/1), micaceous, glauconitic. Sand is very-fine-grained. Contains sparse chalky shell fragments.

Silty sandy clay, micaceous, glauconitic. Sand is fine- to medium-grained. Bioturbated. Contains thin layers of bivalves (Venericardia).

Clayey sandy silt, olive-gray (5Y4/1) to greenish-black (5GY2/1), micaceous, glauconitic. Sand is mediumgrained, occurs in lenses of mostly ( $>80 \%$ ) glauconite. Contains thin layers of bivalves (Venericardia).

\section{Well EEA-M6 (54Q 65)}

Oyster shells (Crassostrea virginica) in matrix of organic-rich silt, grayish-brown (5YR 3/2) to dusky-brown (5YR 2/2). Contains modern roots. Probably fill material.

Clayey silt, moderate-yellowish-brown (10YR 5/4), some mica.

Sand, grayish-orange (10YR 7/4) to dark-yellowish-orange (10YR 6/6), very-fine to medium-grained, poorly sorted, little mica, $<1 \%$ dark minerals. Mottled.

Sand, dark-yellowish-orange (10YR 6/6) to moderate-yellowish-brown (10YR 5/4), which fines downward from fine-grained to coarse-grained at base of interval, medium-sorted, some mica. Contains pebble lag at $5.8 \mathrm{ft}$ and plant material. Wet at $5.3 \mathrm{ft}$.

Clay, pale-yellowish-brown (10YR 6/2) and light-brown (5YR 5/6), plastic. Wavily mottled. Contains carbonate nodules $(0.5-3 \mathrm{~cm})$.

Clay, very-pale-orange (10YR 8/2) to pale-yellowish-brown (10YR 6/2), plastic, no mica, no glauconite.

Massively bedded. Slightly mottled.

Pebbly sand, (10YR 8/6) and very-pale-orange (10YR 8/2) and light-brown (5YR 5/6), fine-grained, no mica, no glauconite. Pebbles are $5-20 \mathrm{~mm}$ in diameter. Mottled.

Sand, very-pale-orange (10YR 8/2), fine-grained, well-sorted, no mica, about $2 \%$ dark minerals. Mottled. Wet. Sand, dark-yellowish-orange (10YR 6/6), fine- to very-fine-grained, well-sorted, no mica, about $1 \%$ dark minerals. Massively bedded. 
Table 2. Lithologic logs of observation wells at the Explosive Experimental Area, Naval Surface Warfare Center, Dahlgren Site, Dahlgren, Virginia-Continued

\begin{tabular}{ll}
\hline Depth (feet) & Description \\
\hline
\end{tabular}

\section{Well EEA-M6 (54Q 65)-Continued}

16.8-17.6 Pebbly sand, (N6), coarse-grained, no mica, no glauconite. Pebbles are 2-10 mm in diameter. Contains thin peaty bed at $16.8 \mathrm{ft}$.

17.6-18.0 Sandy silt, micaceous, no glauconite. Sand is very-fine-grained. Contains plant material, wood fragments.

18.0-20.0 Silty clay, olive-gray (5Y 4/1) to dark-greenish-gray (5GY 4/1), fining downward into clay, dark-greenish-gray (5GY 4/1), plastic, micaceous, no glauconite. Contains plant material, wood fragments.

22.0 Clay, medium-light-gray (N6) and dark-yellowish-orange (10YR 6/6), plastic, little mica, no glauconite. Contains lenses of very-fine- to fine-grained sand. Mottled.

20.0-30.0 Clay, medium-light-gray (N6) and dark-yellowish-orange (10YR 6/6), plastic. Contains abundant wood fragments.

30.0-32.0 Sandy clayey silt, medium-gray (N5), no mica, about $2 \%$ dark minerals. Sand is very-fine-grained. Contains plant material.

30.0-40.0 Clay and coarse-grained sand. Contains abundant plant fragments, possibly from above. Driller noted "harder at $34 \mathrm{ft}$," "softer again" at $38 \mathrm{ft}$.

40.0-40.8 Silty sand, medium-gray (N5), very-fine-grained, micaceous, no glauconite, $<1 \%$ dark minerals.

40.8-42.0 Silty clay, medium-gray (N5), micaceous, no glauconite. Contains black organic stains and sparse shell material.

40.0-50.0 Clay, plastic, and very-fine-grained sand, and pebbles $(2-5 \mathrm{~mm})$, glauconite. One shell fragment noted.

50.0-52.0 Silty clay with lenses of very-fine-grained sand, pale-brown (5YR 5/2), micaceous, organic rich. Contains small (<1 mm) shell/fossil material. Contains plant material.

50.0-60.0 Sandy clay, micaceous, no glauconite. Sand is very-fine- to medium-grained.

60.0-80.0 Silty clay with abundant lenses of very-fine-grained sand, grayish-brown (5YR 3/2), very micaceous, no lauconite, organic-rich. Contains abundant plant material and sparse carbonate fossil material $(<1 \mathrm{~mm})$.

80.0-90.3 Clay and silt thinly interbedded $(1-3 \mathrm{~mm})$ with very-fine-grained sand, dark-greenish-gray (5GY 4/1), micaceous, no glauconite. Contains abundant plant material.

90.3-92.0 Pebbly sand, greenish-black (5GY 2/1), fine- to medium-grained, poorly sorted, no mica, very glauconitic. Pebbles are 2-10 $\mathrm{mm}$ in diameter. Contains wood fragments.

90.0-100.0 Clay, some silt, and sand, very-fine- to coarse-grained, glauconitic. Driller says "out of sand and gravel" at approximately $93.5 \mathrm{ft}$.

$102.0 \quad$ Silty sand, greenish-black (5GY 2/1), very-fine-grained, micaceous, glauconitic.

100.0-110.0 Silty sand, very-fine-grained. Glauconitic.

110.0-112.0 Sand, fine-grained, and clay, interbedded, greenish-black (5GY 2/1), some mica, glauconitic. Heavily bioturbated.

\section{Well EEA-M7 (54Q 67)}

0-3.4 Sandy silt, pale-yellowish-brown (10YR 6/2) to dark-yellowish-orange (10YR 6/6), no mica, no glauconite. Sand is very-fine-grained. Contains modern roots. Not mottled.

3.4-4.0 Silty clay, light-brown (5YR 5/6), no mica, no glauconite. Not mottled. Dry.

4.0-6.0 Sand, dark-yellowish-orange (10YR 6/6) and light-brown (5YR 5/6), very-fine-to fine-grained, no mica, no glauconite. Below $5 \mathrm{ft}$ contains thin $(<2 \mathrm{~mm})$ flasers of mottled clay.

6.0-8.0 Sand, very-pale-orange (10YR 8/2), fine- to very-fine-grained, well-sorted, no mica, no glauconite. Darkyellowish-orange (10YR 6/6) below $7.7 \mathrm{ft}$.

8.0-11.2 Sand, pale-yellowish-brown (10YR 6/2) to dark-yellowish-orange (10YR 6/6), fine-grained, well-sorted. Mottled. Pebble lag at $11.2 \mathrm{ft}$. Wet at approximately $11 \mathrm{ft}$.

11.2-12.0 Silty clayey sand, dusky-yellow-green (5GY 5/2), very-fine- to fine-grained, micaceous, some glauconite. Contains ironstone concretions.

12.0-14.0 Sandy clayey silt, dusky-yellow-green (5GY 5/2), some mica, glauconitic. Sand is very-fine-grained. Contains ironstone concretions.

16.0 Clayey sandy silt, some mica, glauconitic. Sand is very-fine-grained. Contains thin $(<1 \mathrm{~mm})$ lenses of sand.

16.0-18.0 Clayey sandy silt, dusky-yellow-green (5GY 5/2), some mica, glauconitic. Sand is very-fine-grained. Bioturbated. Contains organic material and thin $(<1 \mathrm{~mm})$ lenses of sand. Mottled. Wet at approximately $17 \mathrm{ft}$. 
Table 2. Lithologic logs of observation wells at the Explosive Experimental Area, Naval Surface Warfare Center, Dahlgren Site, Dahlgren, Virginia-Continued

Depth (feet) Description

\section{Well EEA-M7 (54Q 67)-Continued}

18.0-20.0 Clayey sandy silt, dusky-yellow-green (5GY 5/2) and light-brown (5YR 5/6), micaceous, glauconitic. Sand is very-fine-grained. Mottled. Bottom 3 in.: silty clay, dark-greenish-gray (5GY 4/1), micaceous, no glauconite.

20.0-30.0 Silt, some clay, some very-fine-grained sand, dark-greenish-gray (5G 4/1) to greenish-black (5G $2 / 1)$, some mica, glauconitic. Most sand is glauconite. Contains shell fragments.

30.0-32.0 Silty sand, very-fine-grained to fine-grained, and clay, dark-greenish-gray (5G 4/1) to greenish-black (5G $2 / 1)$, micaceous, glauconitic. Clay occurs in flasers and interbeds. Bioturbated. Contains fragmental and whole shells (Macrocallista, Venericardia).

30.0-40.0 Sandy clayey silt, dark-greenish-gray (5G 4/1) to greenish-black (5G 2/1), some mica, glauconitic. Sand is veryfine- to fine-grained. Contains shell fragments.

40.0-42.0 Silty clayey sand, dark-greenish-gray (5G 4/1) to greenish-black (5G $2 / 1)$, very-fine-to fine-grained, some mica, glauconitic. Moderately fossiliferous with chalky bivalves.

40.0-50.0 Sandy clayey silt, dark-greenish-gray (5G 4/1) to greenish-black (5G 2/1), micaceous, glauconitic. Sand is veryfine- to fine-grained. Contains abundant shell fragments. At bottom of interval material becomes clayey silt, dark-greenish-gray (5GY 4/1), less glauconitic.

52.0 Silty clayey sand, greenish-black (5GY 2/1), very-fine-grained, micaceous, very glauconitic. Contains sparse shells and shell fragments.

50.0-60.0 Silt and clay, some sand, dark-greenish-gray (5GY 4/1), some mica, glauconitic. Contains shell fragments.

60.0-62.0 Silty sandy clay, dark-greenish-gray (5GY 4/1), micaceous, glauconitic. Sand is very-fine-to fine-grained. Bioturbated. Contains lenses of sand ( $>80 \%$ glauconite) and thin $(2-4 \mathrm{~cm})$ beds of chalky bivalves (Venericardia).

60.0-65.0 Clayey sandy silt, dark-greenish-gray (5GY 4/1), micaceous, glauconitic. Contains shell fragments.

67.0 Sand, fine- to very-fine-grained, and silty clay, interbedded, dark-greenish-gray (5GY 4/1), micaceous, glauconitic. Bioturbated.

70.0-72.0 Silty clay, with abundant lenses of very-fine- to fine-grained sand, dark-greenish-gray (5GY 4/1), micaceous, glauconitic. Contains moderate amounts of shell material.

\section{Well EEA-M8 (54Q 69)}

0-0.5 Silt, moderate-yellowish-brown (10YR 5/4) to pale-yellowish-brown (10YR 6/2), some mica, no glauconite. Contains modern roots. Contains medium-grained sand bed $(1 \mathrm{~cm}$ thick $)$ at $0.5 \mathrm{ft}$.

0.5-2.0 Clay, light-brown (5YR 5/6) to moderate-yellowish-orange (10YR 6/4), plastic, no mica, no glauconite. Contains organic material. Wavily mottled.

2.0-2.2 Clayey silt, moderate-yellowish-brown (10YR 5/4), little mica, no glauconite. Mottled.

2.2-4.0 Pebbly sand, very-pale-orange(10YR 8/2) to dark-yellowish-orange (10YR 6/6), fine- to coarse-grained, poorly sorted, fining upwards, no mica, no glauconite. Pebbles are $1-5 \mathrm{~mm}$ in diameter.

4.0-6.0 Pebbly sand, light-brown (5YR 5/6), coarse- to fine-grained, poorly sorted, no mica, $<1 \%$ dark minerals. Pebbles are $1-25 \mathrm{~mm}$ in diameter and subrounded. Heavily mottled.

6.0-8.0 Pebbly sand, dark-yellowish-orange (10YR 6/6), coarse- to medium-grained, poorly sorted, no mica, $<1 \%$ dark minerals. Pebbles are 1-20 mm in diameter. Mottled.

8.0-10.0 Pebbly sand, dark-yellowish-orange (10YR 6/6), very-fine-grained, little mica, $<1 \%$ dark minerals. Pebbles are $1-5 \mathrm{~mm}$ in diameter. Medium-bluish-gray (5B 5/1) at bottom.

10.0-12.0 Sand, olive-gray (5Y 4/1), very-fine- to fine-grained, well-sorted, little mica, $<1 \%$ dark minerals. Wet.

12.0-15.2 Sand, dark-greenish-gray (5GY 4/1), very-fine- to fine-grained, well-sorted, no mica, $<1 \%$ dark minerals.

Contains dark organic(?) stains. Contains pebble lag at $15.2 \mathrm{ft}$.

15.2-16.0 Silty clay, dark-greenish-gray ( $5 \mathrm{GY} 4 / 1)$, some mica, no glauconite.

16.0-20.0 Clay, dark-greenish-gray (5GY 4/1), plastic, little mica. Speckled (glauconite? organic material?).

24.0 Clay, dark-greenish-gray (5GY 4/1), plastic, some mica, no glauconite. Contains sparse chalky fossil fragments and plant material.

30.0-32.0 Silty clay with lenses of sand, olive-gray ( $5 \mathrm{Y} 4 / 1)$, some mica, no glauconite. Sand is very-fine-grained. Contains abundant plant material, wood fragments. 
Table 2. Lithologic logs of observation wells at the Explosive Experimental Area, Naval Surface Warfare Center, Dahlgren Site, Dahlgren, Virginia-Continued

Depth (feet) Description

\section{Well EEA-M8 (54Q 69)-Continued}

30.0-40.0 No apparent change

40.0-42.0 Clay, olive-gray (5Y 4/1), plastic, micaceous, speckled. Contains moderate amounts of chalky fossil fragments.

40.0-50.0 No apparent change; some very-fine-grained sand in cuttings

50.0-51.0 Clay, as 40-42 ft interval

51.0-52.0 Sand, olive-gray (5Y 4/1), fine-grained, well-sorted, micaceous, $2-3 \%$ dark minerals (glauconite?).

50.0-60.0 Clay, as 40-50 ft interval. Abundant wood fragments, abundant dark very fine sand (glauconite? organic material?). Little mica. Olive-gray (5Y 4/1) and dark-greenish-gray (5GY 4/1). Driller reports "hit log at approximately $54 \mathrm{ft}$.

60.0-62.0 Sand, very-fine-grained, and sand, medium-grained, and clay, and peat (wood), interbedded, olive-gray (5Y 4/1) and dark-greenish-gray (5GY 4/1), little mica. Pebbles at base of interval.

60.0-70.0 Peat, and sand, very-fine- to fine-grained, and large wood fragments, and pebbles $(1-20 \mathrm{~mm})$.

70.0-72.0 Clay, and sand, medium-grained, and peat, interbedded $(4-6 \mathrm{~cm})$, olive-gray $(5 \mathrm{Y} 4 / 1)$ to dark-greenish-gray (5GY 4/1). Clay has some mica. Sand is glauconitic.

70.0-80.0 Sand and pebbles and wood fragments. Wood is packed with glauconitic fine sand and gravel. Driller notes chatter at $74-78.5 \mathrm{ft}$.

80.0-82.0 Silty sand, dark-greenish-gray (5GY 4/1), very-fine-grained, micaceous, some glauconite. Abundant Macrocallista.

87.0 Sitty sand, dark-greenish-gray ( $5 \mathrm{GY} 4 / 1)$, very-fine-grained, micaceous, some glauconite. Abundant bivalve fragments (Macrocallista). 
Table 3. Depth, lithology, and vertical hydraulic conductivity measurements of selected core intervals at the Explosive Experimental Area, Naval Surface Warfare Center, Dahlgren Site, Dahlgren, Virginia

[Datum is sea level; (-) indicates below sea level; $\mathrm{ft}$-BLS, feet below land surface; $\mathrm{ft} / \mathrm{d}$, foot per day; --, no data]

\begin{tabular}{|c|c|c|c|c|c|c|c|}
\hline $\begin{array}{l}\text { Local } \\
\text { well } \\
\text { no. }\end{array}$ & $\begin{array}{l}\text { Sample } \\
\text { no. }\end{array}$ & $\begin{array}{l}\text { Depth } \\
\text { to top } \\
\text { of in- } \\
\text { terval } \\
\text { (ft-BLS) }\end{array}$ & $\begin{array}{l}\text { Depth } \\
\text { to bot- } \\
\text { tom of } \\
\text { Interval } \\
\text { (ft-BLS) }\end{array}$ & $\begin{array}{l}\text { Altitude } \\
\text { of top } \\
\text { of in- } \\
\text { terval } \\
\text { (feet) }\end{array}$ & $\begin{array}{l}\text { Hydrogeologic unit } \\
\text { of sample }\end{array}$ & $\begin{array}{l}\text { Lithology } \\
\text { conductlvity }\end{array}$ & $\begin{array}{c}\text { Vertical } \\
\text { hydraulic } \\
\text { (ftd) }\end{array}$ \\
\hline EEA-S3 & ST-22 & 8.0 & 9.0 & 0.4 & Columbia aquifer & Sandy clay & $2.27 \times 10^{-3}$ \\
\hline EEA-S6 & ST-14 & 14.0 & 16.0 & .0 & Columbia aquifer & Silty sand & $1.42 \times 10^{-1}$ \\
\hline EEA-S9 & ST-29 & 15.0 & 17.0 & 6.2 & Nanjemoy-Marlboro confining unit & Silty sand & $1.56 \times 10^{-2}$ \\
\hline EEA-S10 & ST-24 & 17.0 & 18.3 & 7.8 & Nanjemoy-Marlboro confining unit & Silty sand & $1.08 \times 10^{-2}$ \\
\hline EEA-S11 & ST-20 & 22.0 & 24.5 & 3.1 & Columbia aquifer & Silty sand & $1.16 \times 10^{-1}$ \\
\hline EEA-S11 & ST-21A & 32.0 & 33.0 & -6.9 & Upper confining unit & Clayey sand & $1.34 \times 10^{-3}$ \\
\hline EEA-S11 & ST-21B & 33.0 & 33.7 & -7.9 & Upper confining unit & Sandy clay & $3.68 \times 10^{-5}$ \\
\hline EEA-S12 & ST-25 & 12.0 & 14.0 & .7 & Columbia aquifer & Silty sand & $8.22 \times 10^{-2}$ \\
\hline EEA-S12 & ST-26A & 20.0 & 21.0 & -7.3 & Upper confining unit & Sandy clay & $2.47 \times 10^{-4}$ \\
\hline EEA-S12 & ST-26B & 21.0 & 21.4 & -8.3 & Upper confining unit & Sandy silt & $1.84 \times 10^{-2}$ \\
\hline EEA-S13 & ST-27 & 35.0 & 37.0 & -11.9 & Upper confining unit & Clay & $4.25 \times 10^{-5}$ \\
\hline EEA-S14 & ST-30 & 20.0 & 21.0 & 2.2 & Upper confining unit & Silty sand & $1.08 \times 10^{-2}$ \\
\hline EEA-S18 & ST-23 & 15.0 & 17.0 & .2 & Nanjemoy-Marlboro confining unit & Silty sand & $2.38 \times 10^{-2}$ \\
\hline EEA-S19 & ST-28 & 13.0 & 15.0 & .2 & Nanjemoy-Marlboro confining unit & Silty sand & $2.72 \times 10^{-2}$ \\
\hline EEA-M1 & ST-17 & 26.5 & 28.5 & -2.1 & Upper confining unit & Clay & $1.30 \times 10^{-3}$ \\
\hline EEA-M1 & ST-18 & 75.0 & 77.0 & -50.6 & Nanjemoy-Marlboro confining unit & Sandy clay & $3.40 \times 10^{-4}$ \\
\hline EEA-M2 & ST-08A & 8.0 & 8.4 & 9.8 & Columbia aquifer & Silty sand & $1.19 \times 10^{-0}$ \\
\hline EEA-M2 & ST-08B & 8.4 & 9.0 & 9.4 & Columbia aquifer & Sand & $4.25 \times 10^{-0}$ \\
\hline EEA-M2 & ST-09 & 18.0 & 20.0 & .2 & Upper confining unit & Sandy clay & $1.59 \times 10^{-4}$ \\
\hline EEA-M2 & ST-10 & 55.0 & 57.0 & -37.2 & Nanjemoy-Marlboro confining unit & Silty sand & $3.68 \times 10^{-2}$ \\
\hline EEA-M2 & ST-11 & 69.5 & 71.5 & -51.7 & Nanjemoy-Marlboro confining unit & Sandy silt & $5.95 \times 10^{-5}$ \\
\hline EEA-M3 & ST-06 & 35.0 & 37.0 & -26.8 & Upper confining unit & Clay & $1.30 \times 10^{-4}$ \\
\hline EEA-M3 & ST-07 & 110.0 & 112.0 & -101.8 & Nanjemoy-Marlboro confining unit & Silty sand & $8.79 \times 10^{-3}$ \\
\hline EEA-M4 & ST-19 & 20.0 & 21.7 & 3.9 & Nanjemoy-Marlboro confining unit & Silty sand & $3.68 \times 10^{-3}$ \\
\hline EEA-M5 & ST-01 & 16.0 & 18.5 & 6.4 & Nanjemoy-Marlboro confining unit & Silty sand & $1.05 \times 10^{-2}$ \\
\hline EEA-M6 & ST-12A & 20.0 & 20.3 & -6.0 & Upper confining unit & Clayey sand & $9.64 \times 10^{-5}$ \\
\hline EEA-M6 & ST-12B & 20.3 & 20.8 & -6.2 & Upper confining unit & Gravelly sand & $7.09 \times 10^{-1}$ \\
\hline EEA-M6 & ST-12C & 20.8 & 21.4 & -6.7 & Upper confining unit & Sandy clay & $5.10 \times 10^{-5}$ \\
\hline EEA-M6 & ST-13A & 100.0 & 100.4 & -85.8 & Nanjemoy-Marlboro confining unit & Sandy gravelly clay & -- \\
\hline EEA-M6 & ST-13B & 100.4 & 101.7 & -86.2 & Nanjemoy-Marlboro confining unit & Silty sand & $7.09 \times 10^{-2}$ \\
\hline EEA-M7 & ST-02 & 5.0 & 7.0 & 7.6 & Columbia aquifer & Silty sand & $2.07 \times 10^{-1}$ \\
\hline EEA-M7 & ST-03 & 14.0 & 16.0 & -1.4 & Nanjemoy-Marlboro confining unit & Silty sand & $5.10 \times 10^{-3}$ \\
\hline EEA-M7 & ST-04 & 50.0 & 52.0 & -37.4 & Nanjemoy-Marlboro confining unit & Clayey sand & $1.42 \times 10^{-3}$ \\
\hline EEA-M7 & ST-05 & 65.0 & 67.0 & -52.4 & Nanjemoy-Marlboro confining unit & Clayey sand & $1.25 \times 10^{-4}$ \\
\hline EEA-M8 & ST-15 & 22.0 & 24.0 & -5.9 & Upper confining unit & Clay & $6.52 \times 10^{-6}$ \\
\hline EEA-M8 & ST-16 & 85.0 & 87.0 & -68.9 & Nanjemoy-Marlboro confining unit & Silty sand & $3.12 \times 10^{-3}$ \\
\hline
\end{tabular}


Table 4. Relative percentage of abundance of minerals in selected core intervals at the Explosive Experimental Area, Naval Surface Warfare Center, Dahlgren Site, Dahlgren, Virginia

[values given in weight percent; --, mineral not found; depth of samples can be found on table 3]

\begin{tabular}{|c|c|c|c|c|c|c|c|c|c|}
\hline Sample & $\begin{array}{l}\text { Local } \\
\text { well } \\
\text { no. }\end{array}$ & Quartz & $\begin{array}{l}\text { Ortho- } \\
\text { clase }\end{array}$ & $\begin{array}{l}\text { Plagio- } \\
\text { clase }\end{array}$ & Illite & Chlorite & Pyrite & Siderite & Calcite \\
\hline ST-22 & EEA-S3 & 96.2 & -- & -- & 3.6 & -- & -- & -- & -- \\
\hline ST-14 & EEA-S6 & 87.3 & 5.5 & 1.2 & 5.9 & -- & -- & -- & -- \\
\hline ST-29 & EEA-S9 & 80.0 & 8.0 & (2) & 9.6 & -- & 2.2 & -- & -- \\
\hline ST-24 & EEA-S10 & 79.1 & 6.7 & (2) & 11.9 & -- & 2.3 & -- & -- \\
\hline ST-20 & EEA-S11 & 71.5 & (2) & 7.1 & 14.3 & 7.1 & -- & -- & -- \\
\hline ST-21A & EEA-S11 & 99.5 & (2) & -- & (2) & -- & -- & -- & -- \\
\hline ST-21B & EEA-S11 & 75.8 & 5.1 & -- & 10.6 & 8.5 & (2) & -- & -- \\
\hline ST-25 & EEA-S12 & 99.8 & (2) & -- & -- & -- & -- & -- & -- \\
\hline ST-26A & EEA-S12 & 99.8 & (2) & -- & -- & -- & -- & -- & -- \\
\hline ST-26B & EEA-S12 & 96.3 & 3.7 & (2) & -- & -- & -- & -- & -- \\
\hline ST-27 & EEA-S13 & 85.9 & 4.0 & 3.6 & 2.4 & 4.1 & -- & -- & -- \\
\hline ST-30 & EEA-S14 & 87.7 & 2.0 & (2) & 9.2 & -- & 1.2 & -- & -- \\
\hline ST-23 & EEA-S18 & 88.9 & 2.7 & -. & 6.2 & -- & 2.1 & -- & -- \\
\hline ST-28 & EEA-S19 & 68.8 & 17.0 & 3.1 & 8.4 & -- & 2.7 & -- & -- \\
\hline ST-17 & EEA-M1 & 79.4 & $-\cdot$ & 4.0 & 11.9 & 4.6 & -- & -- & -- \\
\hline${ }^{1}$ ST-18 & EEA-M1 & 87.4 & -- & -- & 12.5 & -- & (2) & -- & -- \\
\hline ST-08A & EEA-M2 & 93.6 & 2.3 & 4.1 & (2) & (2) & -- & -- & -- \\
\hline ST-08B & EEA-M2 & 99.8 & (2) & (2) & -- & -- & -- & -- & -- \\
\hline ST-09 & EEA-M2 & 91.1 & 2.3 & (2) & 4.2 & 2.4 & -- & -- & -- \\
\hline ST-10 & EEA-M2 & 90.5 & -- & -- & 9.4 & -- & (2) & -- & -- \\
\hline ST-11 & EEA-M2 & 86.2 & -- & -- & 13.8 & -. & (2) & -- & -- \\
\hline ST-06 & EEA-M3 & 60.1 & -- & 6.1 & 15.4 & 8.0 & 10.4 & -- & -- \\
\hline ST-07 & EEA-M3 & 95.1 & -- & -- & 4.9 & -- & -- & -. & -- \\
\hline ST-19 & EEA-M4 & 90.3 & 5.4 & (2) & 3.3 & -- & 1.0 & -- & -- \\
\hline ST-01 & EEA-M5 & 91.3 & 5.1 & (2) & 3.8 & -- & (2) & -- & -- \\
\hline ST-12A & EEA-M6 & 97.1 & (2) & (2) & 2.8 & -- & -- & -- & -- \\
\hline ST-12B & EEA-M6 & 97.3 & (2) & -- & 2.6 & -- & -- & -- & -- \\
\hline ST-12C & EEA-M6 & 88.3 & 3.4 & 4.4 & 2.1 & -- & -- & 5.3 & -- \\
\hline${ }^{1} \mathrm{ST}-13 \mathrm{~A}$ & EEA-M6 & 88.4 & (2) & -- & 11.5 & -- & (2) & -- & -- \\
\hline ST-13B & EEA-M6 & 99.8 & (2) & -- & -- & -- & - & -- & - \\
\hline ST-02 & EEA-M7 & 98.6 & -- & -- & 1.4 & -- & -- & -- & -- \\
\hline ST-03 & EEA-M7 & 87.2 & 5.7 & (2) & 7.2 & -. & -- & -- & -- \\
\hline${ }^{1}$ ST-04 & EEA-M7 & 58.9 & 11.1 & -- & 13.0 & -- & 6.2 & (2) & 10.8 \\
\hline ST-05 & EEA-M7 & 79.8 & 5.6 & -- & 14.2 & -- & (2) & -- & -- \\
\hline ST-15 & EEA-M8 & 91.9 & 5.7 & -- & 2.3 & -- & - & -- & -- \\
\hline ST-16 & EEA-M8 & 81.7 & 2.3 & -- & 15.8 & -. & (2) & -- & -- \\
\hline
\end{tabular}

${ }^{1}$ Mixed-layer clay present.

${ }^{2}$ Trace amounts (less than 1 percent). 
Table 5. Periodic measurements of ground-water levels at the Explosive Experimental Area, Naval Surface Warfare Center, Dahlgren Site, Dahlgren, Virginia, March 1994 through April 1995

[Land-surface datum elevation is in feet above sea level; water levels are in feet above or below (-) sea level]

Local well number: EEA-S1

USGS well number: 54Q56

Land-surface datum elevation: 24.46
Local well number: EEA-S2

USGS well number: 54 Q58

Land-surface datum elevation: 17.89

\begin{tabular}{|c|c|c|c|c|c|c|c|c|c|}
\hline & Date & $\begin{array}{l}\text { Water } \\
\text { level }\end{array}$ & Date & $\begin{array}{l}\text { Water } \\
\text { level }\end{array}$ & & Date & $\begin{array}{l}\text { Water } \\
\text { level }\end{array}$ & Date & $\begin{array}{l}\text { Water } \\
\text { level }\end{array}$ \\
\hline & 1994 & & Aug. & 19.14 & & 1994 & & Dec. 5 & 3.65 \\
\hline Mar. & 17 & 23.27 & Oct. 4 & 18.81 & Mar. & 29 & 11.49 & Dec. & 3.68 \\
\hline Mar. & 23 & 23.38 & Oct. & 18.41 & May & 2 & 7.98 & 1995 & \\
\hline Mar. & 29 & 23.92 & Dec. & 19.19 & May & 31 & 6.75 & Jan. & 3.88 \\
\hline May & 2 & 21.88 & 1995 & & July & 6 & 5.60 & Feb. & 4.08 \\
\hline May & 31 & 20.06 & Jan. $\quad 27$ & 21.03 & Aug. & 3 & 4.91 & Apr. & 4.36 \\
\hline July & 6 & 18.91 & Feb. & 21.61 & Aug. & 29 & 4.46 & & \\
\hline Aug. & 1 & 18.92 & Apr. & 21.03 & Oct. & 4 & 3.82 & & \\
\hline \multicolumn{5}{|c|}{$\begin{array}{l}\text { Local well number: EEA-S3 } \\
\text { USGS well number: } 54 Q 60 \\
\text { Land-surface datum elevation: } 8.36\end{array}$} & \multicolumn{5}{|c|}{$\begin{array}{l}\text { Local well number: EEA-S4 } \\
\text { USGS well number: } 54 Q 62 \\
\text { Land-surface datum elevation: } 23.83\end{array}$} \\
\hline & Date & $\begin{array}{l}\text { Water } \\
\text { level }\end{array}$ & Date & $\begin{array}{l}\text { Water } \\
\text { level }\end{array}$ & & Date & $\begin{array}{l}\text { Water } \\
\text { level }\end{array}$ & Date & $\begin{array}{l}\text { Water } \\
\text { level }\end{array}$ \\
\hline & 1994 & & Aug. 29 & 1.18 & & 1994 & & Aug. 29 & 19.93 \\
\hline Mar. & 16 & 1.11 & Oct. & .78 & Mar. & 17 & 22.73 & Oct. & 19.83 \\
\hline Mar. & 23 & 1.52 & Oct. & 1.53 & Mar. & 23 & 22.99 & Oct. & 19.47 \\
\hline Mar. & 29 & 1.38 & Dec. 20 & .52 & Mar. & 29 & 23.59 & Dec. 20 & 20.10 \\
\hline May & 2 & .27 & 1995 & & May & 2 & 21.81 & 1995 & \\
\hline May & 31 & .86 & Jan. & .48 & May & 31 & 19.92 & Jan. & 22.07 \\
\hline July & 6 & 1.08 & Feb. & .96 & July & 6 & 19.43 & Feb. & 22.46 \\
\hline Aug. & 1 & 1.18 & Apr. & .78 & Aug. & 3 & 22.13 & Apr. & 21.85 \\
\hline
\end{tabular}

Local well number: EEA-S5

USGS well number: 54Q64

Land-surface datum elevation: 22.02
Local well number: EEA-S6

USGS well number: 54Q66

Land-surface datum elevation: 14.05

\begin{tabular}{|c|c|c|c|c|c|c|c|c|}
\hline & Date & $\begin{array}{l}\text { Water } \\
\text { level }\end{array}$ & Date & $\begin{array}{l}\text { Water } \\
\text { level }\end{array}$ & Date & $\begin{array}{l}\text { Water } \\
\text { level }\end{array}$ & Date & $\begin{array}{l}\text { Water } \\
\text { level }\end{array}$ \\
\hline & 1994 & & Dec. 20 & 15.81 & 1994 & & Oct. & 3.33 \\
\hline May & 2 & 21.30 & 1995 & & Mar. 16 & 6.96 & Oct. 25 & 2.88 \\
\hline May & 31 & 18.40 & Jan. 27 & 16.76 & Mar. 23 & 5.87 & Dec. 20 & 3.22 \\
\hline July & 6 & 16.33 & Feb. & 17.38 & Mar. 29 & 11.32 & 1995 & \\
\hline Aug. & 1 & 16.63 & Apr. & 17.53 & May 31 & 3.23 & Jan. 27 & 4.73 \\
\hline Aug. & 29 & 16.16 & & & July 6 & 2.74 & Feb. & 4.55 \\
\hline Oct. & 4 & 16.04 & & & Aug. $\quad 1$ & 3.52 & Apr. & 3.58 \\
\hline Dec. & 5 & 15.82 & & & Aug. 29 & 3.34 & & \\
\hline
\end{tabular}


Table 5. Periodic measurements of ground-water levels at the Explosive Experimental Area, Naval Surface Warfare Center, Dahlgren Site, Dahlgren, Virginia, March 1994 through April 1995-Continued

[Land-surface datum elevation is in feet above sea level; water levels are in feet above or below (-) sea level]

Local well number: EEA-S7

USGS well number: $54 Q 68$

Land-surface datum elevation: 12.45
Local well number: EEA-S8

USGS well number: $54 Q 70$

Land-surface datum elevation: 16.17

\begin{tabular}{|c|c|c|c|c|c|c|c|c|c|}
\hline & Date & $\begin{array}{l}\text { Water } \\
\text { level }\end{array}$ & Date & $\begin{array}{l}\text { Water } \\
\text { level }\end{array}$ & & Date & $\begin{array}{l}\text { Water } \\
\text { level }\end{array}$ & Date & $\begin{array}{l}\text { Water } \\
\text { level }\end{array}$ \\
\hline & 1994 & & Dec. 5 & 0.62 & & 1994 & & Aug. 29 & 8.75 \\
\hline Mar. 2 & 29 & 4.02 & Dec. & .83 & Mar. & 15 & 14.01 & Oct. & 8.65 \\
\hline May & 2 & 2.59 & 1995 & & Mar. & 23 & 13.36 & Oct. & 8.25 \\
\hline May & 31 & 1.87 & Jan. 27 & .87 & Mar. & 29 & 15.16 & Dec. 20 & 8.22 \\
\hline July & 6 & 1.05 & Feb. $\quad 24$ & .85 & May & 2 & 11.22 & 1995 & \\
\hline Aug. & 3 & .71 & Apr. & 1.18 & May & 31 & 9.75 & Jan. & 9.14 \\
\hline Aug. & 29 & .61 & & & July & 6 & 8.89 & Feb. & 9.11 \\
\hline Oct. & 4 & .66 & & & Aug. & 3 & 9.07 & Apr. & 9.11 \\
\hline
\end{tabular}

Local well number: EEA-S9

USGS well number: $54 Q 71$

Land-surface datum elevation: 21.24

Local well number: EEA-S10

USGS well number: 54 Q72

Land-surface datum elevation: 24.76

\begin{tabular}{|c|c|c|c|c|c|c|c|}
\hline Date & $\begin{array}{l}\text { Water } \\
\text { level }\end{array}$ & Date & $\begin{array}{l}\text { Water } \\
\text { level }\end{array}$ & Date & $\begin{array}{l}\text { Water } \\
\text { level }\end{array}$ & Date & $\begin{array}{l}\text { Water } \\
\text { level }\end{array}$ \\
\hline 1994 & & Dec. 5 & 13.47 & 1994 & & Dec. 5 & 18.92 \\
\hline Mar. 29 & 21.24 & Dec. 20 & 13.70 & Mar. 29 & 24.67 & Dec. 20 & 18.85 \\
\hline May 2 & 18.27 & 1995 & & May 2 & 22.76 & 1995 & \\
\hline May 31 & 15.50 & Jan. 27 & 14.52 & May & 20.25 & Jan. $\quad 27$ & 21.63 \\
\hline July 6 & 13.97 & Feb. $\quad 24$ & 14.90 & July & 18.97 & Feb. $\quad 24$ & 22.97 \\
\hline Aug. 3 & 14.74 & Apr. & 15.14 & Aug. 3 & 21.77 & Apr. & 22.03 \\
\hline Aug. 29 & 14.37 & & & Aug. 29 & 19.94 & & \\
\hline Oct. & 13.83 & & & Oct. 4 & 18.67 & & \\
\hline \multicolumn{4}{|c|}{$\begin{array}{l}\text { Local well number: EEA-S11 } \\
\text { USGS well number: } 54 Q 73 \\
\text { Land-surface datum elevation: } 25.14\end{array}$} & \multicolumn{4}{|c|}{$\begin{array}{l}\text { Local well number: EEA-S12 } \\
\text { USGS well number: } 54 Q 74 \\
\text { Land-surface datum elevation: } 12.70\end{array}$} \\
\hline Date & $\begin{array}{l}\text { Water } \\
\text { level }\end{array}$ & Date & $\begin{array}{l}\text { Water } \\
\text { level }\end{array}$ & Date & $\begin{array}{l}\text { Water } \\
\text { level }\end{array}$ & Date & $\begin{array}{l}\text { Water } \\
\text { level }\end{array}$ \\
\hline 1994 & & Dec. 5 & 18.45 & 1994 & & Dec. & 5.18 \\
\hline Mar. 29 & 23.80 & Dec. 20 & 18.50 & Mar. 29 & 7.82 & Dec. 20 & 5.20 \\
\hline May & 21.67 & 1995 & & May & 6.35 & 1995 & \\
\hline May 31 & 20.62 & Jan. $\quad 27$ & 19.34 & May & 5.71 & Jan. & 5.41 \\
\hline July & 17.58 & Feb. & 19.62 & July & 5.00 & Feb. & 5.35 \\
\hline Aug. & 17.54 & Apr. & 19.54 & Aug. & 5.05 & Apr. & 5.30 \\
\hline Aug. 29 & 17.66 & & & Aug. 29 & 4.99 & & \\
\hline Oct. $\quad 4$ & 17.44 & & & Oct. 4 & 4.93 & & \\
\hline
\end{tabular}


Table 5. Periodic measurements of ground-water levels at the Explosive Experimental Area, Naval Surface Warfare Center, Dahlgren Site, Dahlgren, Virginia, March 1994 through April 1995-Continued

[Land-surface datum elevation is in feet above sea level; water levels are in feet above or below (-) sea level]

Local well number: EEA-S13

USGS well number: $54 Q 75$

Land-surface datum elevation: 23.11
Local well number: EEA-S14

USGS well number: 54 Q76

Land-surface datum elevation: 22.20

\begin{tabular}{|c|c|c|c|c|c|c|c|c|}
\hline & Date & $\begin{array}{l}\text { Water } \\
\text { level }\end{array}$ & Date & $\begin{array}{l}\text { Water } \\
\text { level }\end{array}$ & Date & $\begin{array}{l}\text { Water } \\
\text { level }\end{array}$ & Date & $\begin{array}{l}\text { Water } \\
\text { level }\end{array}$ \\
\hline & 1994 & & Dec. 5 & 6.65 & 1994 & & Dec. 5 & 7.09 \\
\hline Mar. & 29 & 8.70 & Dec. 20 & 6.65 & Mar. 29 & 18.20 & Dec. 20 & 7.08 \\
\hline May & 2 & 8.39 & 1995 & & May 2 & 13.34 & 1995 & \\
\hline May & 31 & 7.90 & Jan. 27 & 6.69 & May 31 & 11.63 & Jan. 27 & 7.20 \\
\hline July & 6 & 7.30 & Feb. $\quad 24$ & 6.73 & July 6 & 9.89 & Feb. $\quad 24$ & 7.41 \\
\hline Aug. & 1 & 7.11 & Apr. 3 & 6.82 & Aug. & 7.53 & Apr. 3 & 7.89 \\
\hline Aug. & 29 & 6.91 & & & Aug. 29 & 7.76 & & \\
\hline Oct. & 4 & 6.65 & & & Oct. 4 & 6.35 & & \\
\hline \multicolumn{5}{|c|}{$\begin{array}{l}\text { Local well number: EEA-S15 } \\
\text { USGS well number: 54Q77 } \\
\text { Land-surface datum elevation: } 21.73\end{array}$} & \multicolumn{4}{|c|}{$\begin{array}{l}\text { Local well number: EEA-S16 } \\
\text { USGS well number: } 54 Q 78 \\
\text { Land-surface datum elevation: } 16.33\end{array}$} \\
\hline & Date & $\begin{array}{l}\text { Water } \\
\text { level }\end{array}$ & Date & $\begin{array}{l}\text { Water } \\
\text { level }\end{array}$ & Date & $\begin{array}{l}\text { Water } \\
\text { level }\end{array}$ & Date & $\begin{array}{l}\text { Water } \\
\text { level }\end{array}$ \\
\hline & 1994 & & Dec. 5 & -0.05 & 1994 & & Dec. 5 & 9.17 \\
\hline Mar. & 29 & 9.63 & Dec. 20 & .26 & Mar. 29 & 16.03 & Dec. 20 & 9.32 \\
\hline May & 2 & 9.40 & 1995 & & May 2 & 12.93 & 1995 & \\
\hline May & 31 & 7.04 & Jan. 27 & .85 & May 31 & 10.68 & Jan. 27 & 10.87 \\
\hline July & 6 & 3.38 & Feb. $\quad 24$ & 1.15 & July 6 & 8.85 & Feb. $\quad 24$ & 11.35 \\
\hline Aug. & 1 & 1.55 & Apr. 3 & 1.46 & Aug. & 9.17 & Apr. 3 & 10.82 \\
\hline Aug. & 29 & .97 & & & Aug. 29 & 9.09 & & \\
\hline Oct. & 4 & -.64 & & & Oct. 4 & 8.73 & & \\
\hline \multicolumn{5}{|c|}{$\begin{array}{l}\text { Local well number: EEA-S17 } \\
\text { USGS well number: 54Q79 } \\
\text { Land-surface datum elevation: } 11.43\end{array}$} & \multicolumn{4}{|c|}{$\begin{array}{l}\text { Local well number: EEA-S18 } \\
\text { USGS well number: } 54 Q 80 \\
\text { Land-surface datum elevation: } 15.20\end{array}$} \\
\hline & Date & $\begin{array}{l}\text { Water } \\
\text { level }\end{array}$ & Date & $\begin{array}{l}\text { Water } \\
\text { level }\end{array}$ & Date & $\begin{array}{l}\text { Water } \\
\text { level }\end{array}$ & Date & $\begin{array}{l}\text { Water } \\
\text { level }\end{array}$ \\
\hline & 1994 & & Dec. 5 & 3.39 & 1994 & & Dec. 5 & 11.50 \\
\hline Mar. 2 & 29 & 9.81 & Dec. 20 & 3.35 & Mar. 29 & 13.41 & Dec. 20 & 10.99 \\
\hline May & 2 & 2.98 & 1995 & & May 2 & 11.62 & 1995 & \\
\hline May & 31 & 2.57 & Jan. 27 & 3.86 & May & 10.75 & Jan. 27 & 11.42 \\
\hline July & 6 & 1.80 & Feb. $\quad 24$ & 3.61 & July & 9.79 & Feb. $\quad 24$ & 11.45 \\
\hline Aug. & 1 & 2.13 & Apr. 3 & 3.08 & Aug. 3 & 11.95 & Apr. 3 & 11.21 \\
\hline Aug. & 29 & 2.94 & & & Aug. 29 & 10.08 & & \\
\hline Oct. & 4 & 3.15 & & & Oct. 4 & 10.18 & & \\
\hline
\end{tabular}


Table 5. Periodic measurements of ground-water levels at the Explosive Experimental Area, Naval Surface Warfare Center, Dahlgren Site, Dahlgren, Virginia, March 1994 through April 1995-Continued

[Land-surface datum elevation is in feet above sea level; water levels are in feet above or below (-) sea level]

Local well number: EEA-S19

USGS well number: 54Q81

Land-surface datum elevation: 13.21
Local well number: EEA-S20

USGS well number: 54Q82

Land-surface datum elevation: 11.81

\begin{tabular}{|c|c|c|c|c|c|c|c|c|}
\hline & Date & $\begin{array}{l}\text { Water } \\
\text { level }\end{array}$ & Date & $\begin{array}{l}\text { Water } \\
\text { level }\end{array}$ & Date & $\begin{array}{l}\text { Water } \\
\text { level }\end{array}$ & Date & $\begin{array}{l}\text { Water } \\
\text { level }\end{array}$ \\
\hline & 1994 & & Dec. 20 & 10.11 & 1994 & & Dec. & 9.53 \\
\hline May & 2 & 11.17 & 1995 & & Mar. 29 & 11.43 & Dec. & 8.12 \\
\hline May & 31 & 10.65 & Jan. 27 & 10.31 & May & 9.22 & 1995 & \\
\hline July & 6 & 10.60 & Feb. $\quad 24$ & 10.06 & May & 8.36 & Jan. & 8.65 \\
\hline Aug. & 3 & 11.60 & Apr. & 9.93 & July & 7.78 & Feb. & 8.39 \\
\hline Aug. & 29 & 10.67 & & & Aug. & 10.64 & Apr. & 7.86 \\
\hline Oct. & 4 & 10.57 & & & Aug. & 8.21 & & \\
\hline Dec. & 5 & 10.23 & & & Oct. & 8.11 & & \\
\hline
\end{tabular}

Local well number: EEA-M1

USGS well number: 54Q55

Land-surface datum elevation: 24.44

Local well number: EEA-M2

USGS well number: 54Q57

Land-surface datum elevation: 17.77

\begin{tabular}{|c|c|c|c|c|c|c|c|c|}
\hline & Date & $\begin{array}{l}\text { Water } \\
\text { level }\end{array}$ & Date & $\begin{array}{l}\text { Water } \\
\text { level }\end{array}$ & Date & $\begin{array}{l}\text { Water } \\
\text { level }\end{array}$ & Date & $\begin{array}{l}\text { Water } \\
\text { level }\end{array}$ \\
\hline & 1994 & & Aug. 29 & 14.52 & 1994 & & Dec. & 5.04 \\
\hline Mar. & 17 & 16.73 & Oct. 4 & 14.06 & Mar. 29 & 6.33 & Dec. & 5.03 \\
\hline Mar. & 23 & 16.75 & Oct. 25 & 13.90 & May & 6.03 & 1995 & \\
\hline Mar. & 29 & 16.92 & Dec. 20 & 13.85 & May 31 & 5.71 & Jan. $\quad 27$ & 5.09 \\
\hline May & 2 & 16.56 & 1995 & & July & 5.42 & Feb. & 5.12 \\
\hline May & 31 & 16.00 & Jan. & 14.22 & Aug. & 5.35 & Apr. & 5.09 \\
\hline July & 6 & 15.00 & Feb. & 14.69 & Aug. 29 & 5.21 & & \\
\hline Aug. & 1 & 14.55 & Apr. & 15.06 & Oct. & 5.03 & & \\
\hline \multicolumn{5}{|c|}{$\begin{array}{l}\text { Local well number: EEA-M3 } \\
\text { USGS well number: } 54 \text { Q599 } \\
\text { Land-surface datum elevation: } 8.16\end{array}$} & \multicolumn{4}{|c|}{$\begin{array}{l}\text { Local well number: EEA-M4 } \\
\text { USGS well number: } 54 \text { Q } 61 \\
\text { Land-surface datum elevation: } 23.94\end{array}$} \\
\hline & Date & $\begin{array}{l}\text { Water } \\
\text { level }\end{array}$ & Date & $\begin{array}{l}\text { Water } \\
\text { level }\end{array}$ & Date & $\begin{array}{l}\text { Water } \\
\text { level }\end{array}$ & Date & $\begin{array}{l}\text { Water } \\
\text { level }\end{array}$ \\
\hline & 1994 & & Aug. 29 & 1.85 & 1994 & & Aug. 29 & 19.27 \\
\hline Mar. & 16 & 1.84 & Oct. 4 & 1.62 & Mar. 17 & 23.26 & Oct. & 19.09 \\
\hline Mar. & 23 & 2.12 & Oct. & 2.03 & Mar. 23 & 23.30 & Oct. & 18.73 \\
\hline Mar. & 29 & 1.76 & Dec. 20 & 1.42 & Mar. 29 & 23.74 & Dec. & 19.34 \\
\hline May & 2 & 1.46 & 1995 & & May & 22.04 & 1995 & \\
\hline May & 31 & 1.77 & Jan. & 1.30 & May 31 & 19.97 & Jan. & 21.71 \\
\hline July & 6 & 1.85 & Feb. & 1.64 & July & 18.69 & Feb. & 22.55 \\
\hline Aug. & 1 & 1.84 & Apr. & 1.57 & Aug. & 20.06 & Apr. & 21.89 \\
\hline
\end{tabular}


Table 5. Periodic measurements of ground-water levels at the Explosive Experimental Area, Naval Surface Warfare Center, Dahlgren Site, Dahlgren, Virginia, March 1994 through April 1995-Continued

[Land-surface datum elevation is in feet above sea level; water levels are in feet above or below (-) sea level]

Local well number: EEA-M5

USGS well number: 54 Q63

Land-surface datum elevation: 22.42
Local well number: EEA-M6

USGS well number: 54 Q65

Land-surface datum elevation: 14.15

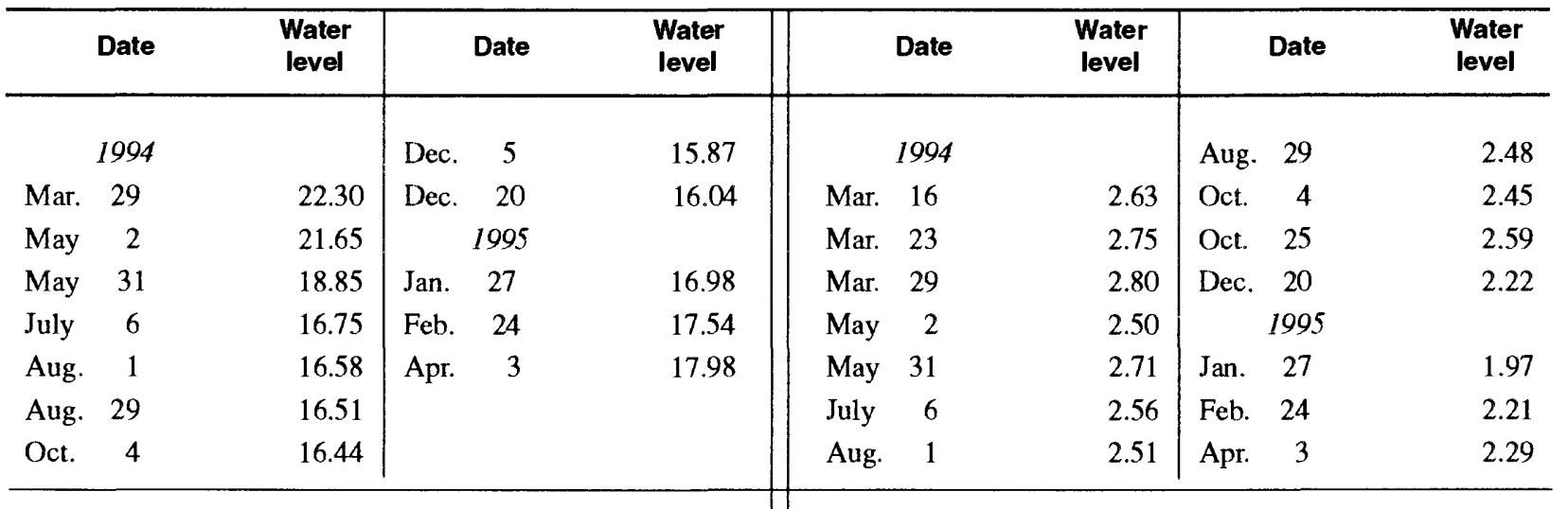

Local well number: EEA-M7

USGS well number: 54Q67

Land-surface datum elevation: 12.61

Local well number: EEA-M8

USGS well number: 54Q69

Land-surface datum elevation: 16.12

\begin{tabular}{|c|c|c|c|c|c|c|c|c|c|}
\hline & Date & $\begin{array}{l}\text { Water } \\
\text { level }\end{array}$ & Date & $\begin{array}{l}\text { Water } \\
\text { level }\end{array}$ & & Date & $\begin{array}{l}\text { Water } \\
\text { level }\end{array}$ & Date & $\begin{array}{l}\text { Water } \\
\text { level }\end{array}$ \\
\hline & 1994 & & Dec. 5 & 0.62 & & 1994 & & Aug. & 3.24 \\
\hline Mar. & 29 & 1.96 & Dec. 20 & .68 & Mar. & 15 & 3.08 & Oct. & 2.96 \\
\hline May & 2 & 2.15 & 1995 & & Mar. & 23 & 3.53 & Oct. & 3.11 \\
\hline May & 31 & 1.88 & Jan. 27 & .73 & Mar. & 29 & 3.20 & Dec. & 2.69 \\
\hline July & 6 & -3.91 & Feb. & .83 & May & 2 & 2.95 & 1995 & \\
\hline Aug. & 3 & .91 & Apr. & .89 & May & 31 & 3.58 & Jan. & 2.85 \\
\hline Aug. 2 & 29 & .78 & & & July & 6 & 3.01 & Feb. & 2.92 \\
\hline Oct. & 4 & .66 & & & Aug. & 3 & 3.12 & Apr. & 2.71 \\
\hline
\end{tabular}


Table 6. Slug-test data for observation well EEA-S1 at the Explosive Experimental Area, Naval Surface Warfare Center, Dahlgren Site, Dahlgren, Virginia ${ }^{1}$

[For location of observation well, see plate 1]

\section{Well EEA-S1}

Static water level: 9.67 feet below measuring point

Date: March 10, 1994

\begin{tabular}{|c|c|}
\hline $\begin{array}{c}\text { Elapsed } \\
\text { time } \\
\text { (mlnutes) }\end{array}$ & $\begin{array}{c}\text { Depth of water } \\
\text { below static } \\
\text { water level } \\
\text { (feet) }\end{array}$ \\
\hline 0.0034 & 1.98 \\
\hline .0067 & 1.98 \\
\hline .0100 & 1.98 \\
\hline .0134 & 1.98 \\
\hline .0167 & 1.97 \\
\hline .0200 & 1.97 \\
\hline .0234 & 1.97 \\
\hline .0267 & 1.97 \\
\hline .0300 & 1.97 \\
\hline .0334 & 1.97 \\
\hline .0367 & 1.97 \\
\hline .0400 & 1.96 \\
\hline .0434 & 1.96 \\
\hline .0467 & 1.96 \\
\hline .0500 & 1.96 \\
\hline .0534 & 1.96 \\
\hline .0567 & 1.96 \\
\hline .0600 & 1.96 \\
\hline .0634 & 1.96 \\
\hline .0667 & 1.96 \\
\hline .0700 & 1.96 \\
\hline .0734 & 1.96 \\
\hline .0767 & 1.96 \\
\hline .0800 & 1.95 \\
\hline .0834 & 1.95 \\
\hline .0867 & 1.95 \\
\hline .0900 & 1.95 \\
\hline .0934 & 1.95 \\
\hline .0967 & 1.95 \\
\hline .1000 & 1.95 \\
\hline .1034 & 1.95 \\
\hline .1067 & 1.95 \\
\hline
\end{tabular}

${ }^{1}$ The remaining pages of table 6 are stored on diskette, located at the back of this report. 
Table 7. Reporting units and limits for selected water-quality constituents

$\left[{ }^{\circ} \mathrm{C}\right.$, degrees Celsius; $\mu \mathrm{g} / \mathrm{L}$, micrograms per liter; $\mathrm{mg} / \mathrm{L}$, milligrams per liter, $\mu \mathrm{S} / \mathrm{cm}$, microsiemens per centimeter at $\left.25^{\circ} \mathrm{C}\right]$

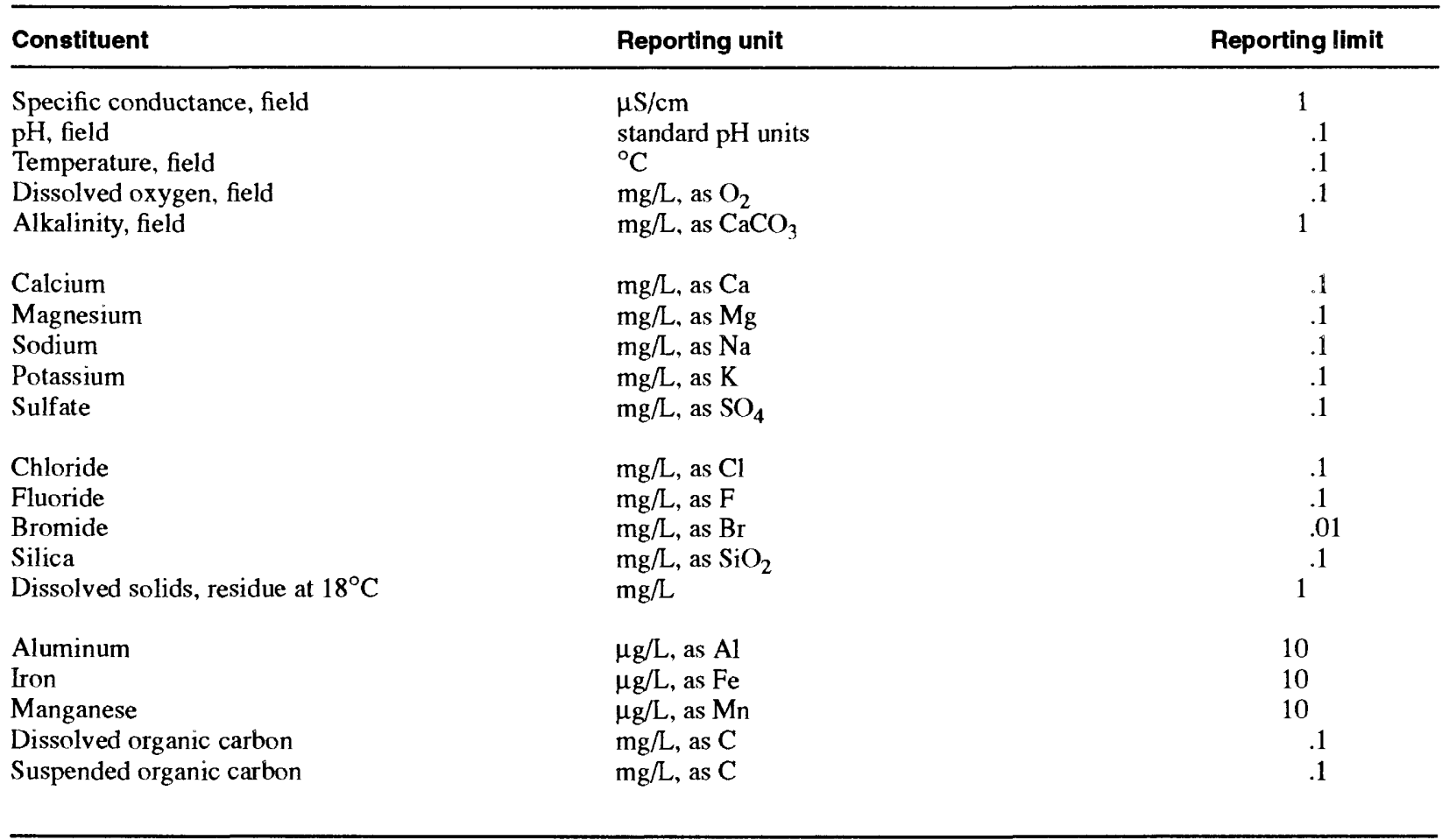

Table 8. Concentrations of major dissolved constituents in surface water from the Explosive Experimental Area, Naval Surface Warfare Center, Dahlgren Site, Dahlgren, Virginia

$\left[{ }^{\circ} \mathrm{C}\right.$, degrees Celsius; $\mu \mathrm{g} / \mathrm{L}$, micrograms per liter, mg/L, milligrams per liter, $\mu \mathrm{S} / \mathrm{cm}$, microsiemens per centimeter at $25^{\circ} \mathrm{C} ;>$, greater than; $<$, less than; for surface-water sample locations, see plate 1]

\begin{tabular}{|c|c|c|c|c|c|c|c|c|c|c|c|}
\hline $\begin{array}{l}\text { Local } \\
\text { well } \\
\text { no. }\end{array}$ & Date & $\begin{array}{c}\text { Specific } \\
\text { conduct } \\
\text { ance, } \\
\text { field } \\
(\mu \mathrm{S} / \mathrm{cm})\end{array}$ & $\begin{array}{l}c \\
t- \\
\text { ) }\end{array}$ & $\begin{array}{l}\text { Temp } \\
\text { atur } \\
\text { fiel } \\
{ }^{\circ} \mathrm{C}\end{array}$ & & $\begin{array}{l}\text { Oxygen, } \\
\text { lissolved, } \\
\text { field } \\
(\mathrm{mg} / \mathrm{L}, \\
\left.\text { as } \mathrm{O}_{2}\right)\end{array}$ & $\begin{array}{l}\text { Calcium } \\
\text { (mg/l, } \\
\text { as Ca) }\end{array}$ & $\begin{array}{l}\text { Magne- } \\
\text { sium } \\
(\mathrm{mg} / \mathrm{L} \text {, } \\
\text { as Mg) }\end{array}$ & $\begin{array}{l}\text { Sodium } \\
\text { (mg/L, } \\
\text { as Na) }\end{array}$ & $\begin{array}{l}\text { Potas- } \\
\text { sium } \\
\text { (mg/L, } \\
\text { as K) }\end{array}$ & $\begin{array}{l}\text { Alka- } \\
\text { linity, } \\
\text { field } \\
\left(\mathrm{mg}^{\prime} \mathrm{L}, \text { as }\right. \\
\left.\mathrm{CaCO}_{3}\right)\end{array}$ \\
\hline EEA-SW1 & $07-20-94$ & 7,440 & 6.0 & 29. & & 5.4 & 66 & 150 & 1,300 & 49 & 26 \\
\hline EEA-SW2 & $07-20-94$ & 9,200 & 7.0 & 30. & & 6.5 & 79 & 190 & 1,500 & 63 & 30 \\
\hline${ }^{1}$ EEA-SW2 & $07-20-94$ & 9,200 & 7.0 & 30. & & 6.5 & 77 & 190 & 1,500 & 64 & 52 \\
\hline EEA-SW3 & $07-20-94$ & 9.520 & 6.4 & 27. & & 3.6 & 84 & 200 & 1,600 & 61 & 22 \\
\hline $\begin{array}{l}\text { Local } \\
\text { well } \\
\text { no. }\end{array}$ & $\begin{array}{l}\text { Sulfate } \\
\text { (mg/L, } \\
\text { as } \\
\left.\mathrm{SO}_{4}\right)\end{array}$ & $\begin{array}{l}\text { Chloride } \\
\text { (mg/L, } \\
\text { as Cl) }\end{array}$ & $\begin{array}{c}\text { Fluoride } \\
\text { (mg/L, } \\
\text { as F) }\end{array}$ & $\begin{array}{c}\text { Bromide } \\
\text { (mg/L, } \\
\text { as Br }\end{array}$ & $\begin{array}{c}\text { Silica } \\
\text { (mg/L, } \\
\text { as } \\
\left.\mathrm{SiO}_{2}\right)\end{array}$ & $\begin{array}{c}\text { Solids, } \\
\text { dlssolved } \\
\text { (residueat } \\
180^{\circ} \mathrm{C} \text { ) }\end{array}$ & $\begin{array}{l}\text { Alumi- } \\
\text { num } \\
\text { t } \mu \mathrm{g} / \mathrm{L}, \\
\text { as Al) }\end{array}$ & $\begin{array}{l}\text { Iron } \\
(\mu \mathrm{g} / \mathrm{L}, \\
\text { as Fe })\end{array}$ & $\begin{array}{c}\text { Manga- } \\
\text { nese } \\
(\mu \mathrm{g} / \mathrm{L}, \\
\text { as } \mathrm{Mn})\end{array}$ & $\begin{array}{l}\text { Carbon, } \\
\text { organlc, } \\
\text { dissolved } \\
\text { (mglL, } \\
\text { as C) }\end{array}$ & $\begin{array}{c}\text { Carbon, } \\
\text { organic, } \\
\text { suspended } \\
\text { (mg/L, as C) }\end{array}$ \\
\hline EEA-SWl & 320 & 2,200 & .20 & 7.6 & 8.7 & 4,430 & $<10$ & 20 & 50 & 4.2 & $>3.3$ \\
\hline EEA-SW2 & 400 & 2,800 & .30 & 9.8 & 6.1 & 5,410 & 10 & 30 & 20 & 3.9 & .8 \\
\hline${ }^{1}$ EEA-SW2 & 400 & 2,800 & .30 & 9.7 & 6.0 & 5,410 & $<10$ & 20 & 10 & 2.9 & .7 \\
\hline EEA-SW3 & 410 & 2,900 & .30 & 10 & 2.3 & 5,530 & $<10$ & 40 & 90 & 6.0 & 1.4 \\
\hline
\end{tabular}

${ }^{1}$ Duplicate sample. 
Table 9. Concentrations of major dissolved constituents in ground water from the Explosive Experimental Area, Naval Surface Warfare Center, Dahlgren Site, Dahlgren, Virginia

$\left[{ }^{\circ} \mathrm{C}\right.$, degrees Celsius; $\mu \mathrm{g} / \mathrm{L}$, micrograms per liter; $\mathrm{mg} / \mathrm{L}$, milligrams per liter, $\mu \mathrm{S} / \mathrm{cm}$, microsiemens per centimeter at $25^{\circ} \mathrm{C}$;

--, data not collected; >, greater than; <, less than; for locations of observation wells, see plate 1]

\begin{tabular}{|c|c|c|c|c|c|c|c|c|c|c|}
\hline $\begin{array}{c}\text { Local } \\
\text { well } \\
\text { no. }\end{array}$ & $\begin{array}{c}\text { USGS } \\
\text { well } \\
\text { no. }\end{array}$ & Date & $\begin{array}{l}\text { Specific } \\
\text { conduct- } \\
\text { ance, } \\
\text { field } \\
(\mu S / \mathrm{cm})\end{array}$ & $\begin{array}{c}\text { pH, } \\
\text { fieid } \\
\text { (standard } \\
\text { units) }\end{array}$ & $\begin{array}{l}\text { Temper- } \\
\text { ature, } \\
\text { field } \\
\left({ }^{\circ} \mathrm{C}\right)\end{array}$ & $\begin{array}{c}\text { Oxygen, } \\
\text { dissolved, } \\
\text { field } \\
(\mathrm{mg} / \mathrm{h}, \\
\left.\text { as } \mathrm{O}_{2}\right)\end{array}$ & $\begin{array}{c}\text { Calcium } \\
\text { (mg/L, } \\
\text { as Ca) }\end{array}$ & $\begin{array}{c}\text { Magne- } \\
\text { sium } \\
\text { (mg/L, } \\
\text { as } \\
\text { Mg) }\end{array}$ & $\begin{array}{l}\text { Sodium } \\
\text { (mg/L, } \\
\text { as } \mathrm{Na} \text { ) }\end{array}$ & $\begin{array}{c}\text { Potas- } \\
\text { sium } \\
(\mathrm{mg} / \mathrm{L} \text {, } \\
\text { as K) }\end{array}$ \\
\hline EEA-S1 & $54 \mathrm{Q} 56$ & $06-22-94$ & 225 & 5.9 & 14.0 & 4.3 & 13 & 3.9 & 9.3 & 2.7 \\
\hline EEA-S2 & $54 \mathrm{Q} 58$ & $06-27-94$ & 65 & 5.0 & 14.5 & 5.8 & 3.4 & 1.8 & 2.2 & $<.10$ \\
\hline EEA-S3 & $54 \mathrm{Q} 60$ & $06-14-94$ & 7,600 & 6.4 & 13.0 & .1 & 220 & 160 & 1,100 & 27 \\
\hline EEA-S4 & $54 \mathrm{Q} 62$ & $07-05-94$ & 300 & 6.3 & 16.0 & 1.8 & 46 & 2.1 & 8.4 & 2.8 \\
\hline EEA-S5 & $54 \mathrm{Q} 64$ & $06-29-94$ & 151 & 5.2 & 15.0 & 5.2 & 6.7 & 2.2 & 14 & .60 \\
\hline EEA-S6 & $54 \mathrm{Q} 66$ & $06-17-94$ & 258 & 6.0 & -- & -. & 17 & 1.2 & 24 & 4.0 \\
\hline EEA-S7 & $54 Q 68$ & $06-30-94$ & 137 & 5.1 & 16.0 & 4.3 & 6.7 & 2.4 & 11 & .90 \\
\hline EEA-S8 & $54 \mathrm{Q} 70$ & $06-23-94$ & 169 & 4.2 & 14.0 & 7.4 & 12 & 3.0 & 6.2 & 3.7 \\
\hline${ }^{1}$ EEA-S8 & $54 \mathrm{Q} 70$ & $06-23-94$ & 170 & 4.2 & 14.0 & 7.4 & 12 & 3.0 & 6.1 & 3.8 \\
\hline EEA-S9 & $54 \mathrm{Q} 71$ & $06-20-94$ & 315 & 6.5 & 13.5 & 8.2 & 44 & 3.5 & 9.3 & 4.6 \\
\hline EEA-S10 & $54 \mathrm{Q} 72$ & $06-20-94$ & 680 & 6.8 & 13.0 & -- & 44 & 16 & 82 & 2.9 \\
\hline EEA-S11 & $54 \mathrm{Q} 73$ & $06-24-94$ & 88 & 6.0 & 12.5 & .3 & 5.3 & .82 & 3.1 & 1.0 \\
\hline EEA-S 12 & $54 \mathrm{Q} 74$ & $06-28-94$ & 63 & 6.0 & 14.0 & 3.8 & 4.8 & .91 & 1.8 & .40 \\
\hline EEA-S13 & $54 Q 75$ & $06-28-94$ & 143 & 5.9 & 12.0 & .2 & 14 & 2.5 & 2.9 & 1.3 \\
\hline EEA-S14 & $54 Q 76$ & $06-15-94$ & 103 & 5.0 & 12.0 & .9 & 17 & 2.1 & 2.3 & 1.0 \\
\hline EEA-S15 & $54 \mathrm{Q} 77$ & $06-15-94$ & 215 & 4.5 & 12.0 & 7.2 & 17 & 3.4 & 4.9 & 2.7 \\
\hline EEA-S 16 & $54 Q 78$ & $06-16-94$ & 88 & 5.0 & 13.5 & 3.0 & 1.4 & 1.1 & 11 & 1.6 \\
\hline EEA-S17 & $54 \mathrm{Q} 79$ & $06-16-94$ & 56 & 5.6 & 13.5 & 9.0 & 2.6 & .86 & 4.2 & .60 \\
\hline EEA-S18 & $54 \mathrm{Q} 80$ & $06-21-94$ & 197 & 6.3 & 13.0 & 7.7 & 27 & 2.5 & 6.2 & 1.9 \\
\hline EEA-S19 & $54 \mathrm{Q} 81$ & $06-21-94$ & 616 & 6.3 & 15.0 & 5.6 & 96 & 5.3 & 3.8 & 6.2 \\
\hline EEA-S20 & $54 \mathrm{Q} 82$ & $06-24-94$ & 48 & 5.2 & 14.0 & .6 & 1.5 & .97 & 3.0 & 1.2 \\
\hline EEA-M1 & $54 \mathrm{Q} 55$ & $06-22-94$ & 338 & 6.4 & 15.0 & .3 & 19 & 7.0 & 34 & 10 \\
\hline EEA-M2 & $54 \mathrm{Q} 57$ & $06-27-94$ & 535 & 7.9 & 15.0 & .3 & 33 & 5.3 & 76 & 11 \\
\hline EEA-M3 & $54 \mathrm{Q} 59$ & $06-14-94$ & 600 & 6.9 & 15.0 & .5 & 30 & 14 & 66 & 12 \\
\hline EEA-M4 & $54 \mathrm{Q} 61$ & $07-05-94$ & 575 & 6.9 & 15.0 & 2.3 & 34 & 15 & 65 & 12 \\
\hline EEA-M5 & $54 Q 63$ & $06-30-94$ & 620 & 8.1 & 14.0 & 6.0 & 21 & 9.0 & 100 & 18 \\
\hline EEA-M6 & $54 \mathrm{Q} 65$ & $06-17-94$ & 595 & 6.6 & 15.0 & .2 & 39 & 11 & 66 & 10 \\
\hline${ }^{1}$ EEA-M6 & $54 \mathrm{Q} 65$ & $06-17-94$ & 592 & 6.5 & 15.0 & .2 & 39 & 11 & 66 & 10 \\
\hline EEA-M7 & $54 \mathrm{Q} 67$ & $06-30-94$ & 1,330 & 6.6 & -- & -. & 140 & 59 & 57 & 30 \\
\hline EEA-M8 & $54 \mathrm{Q} 69$ & $06-23-94$ & 552 & 6.6 & 14.5 & .5 & 27 & 6.9 & 78 & 7.7 \\
\hline
\end{tabular}

${ }^{1}$ Duplicate sample. 
Table 9. -Continued

\begin{tabular}{|c|c|c|c|c|c|c|c|c|c|c|c|}
\hline $\begin{array}{c}\text { Alka- } \\
\text { linity, } \\
\text { field } \\
\left(\mathrm{mg}^{2} \text {, as }\right. \\
\left.\mathrm{CaCO}_{3}\right)\end{array}$ & $\begin{array}{c}\text { Sulfate } \\
\text { (mg/L, } \\
\text { as } \\
\left.\mathrm{sO}_{4}\right)\end{array}$ & $\begin{array}{l}\text { Chloride } \\
\text { (mg/L, } \\
\text { as Cl) }\end{array}$ & $\begin{array}{c}\text { Fluoride } \\
\text { (mg/L, } \\
\text { as F) }\end{array}$ & $\begin{array}{c}\text { Sillica } \\
\left(\mathrm{mg}^{\prime} \mathrm{L},\right. \\
\left.\text { as } \mathrm{SiO}_{2}\right)\end{array}$ & $\begin{array}{l}\text { Solids, } \\
\text { dissolved } \\
\text { (residue } \\
\text { at } 180^{\circ} \mathrm{C} \text { ) }\end{array}$ & $\begin{array}{l}\text { Alumi- } \\
\text { num } \\
\text { ( } \mu \mathrm{g} / \mathrm{L}, \\
\text { as AI) }\end{array}$ & $\begin{array}{c}\text { Iron } \\
(\mu g / L, \\
\text { as } \mathrm{Fe})\end{array}$ & $\begin{array}{l}\text { Manga- } \\
\text { nese } \\
(\mu g / L, \\
\text { as Mn) }\end{array}$ & $\begin{array}{l}\text { Carbon, } \\
\text { organlc, } \\
\text { dissolved } \\
\text { (mg/L, } \\
\text { as C) }\end{array}$ & $\begin{array}{l}\text { Carbon, } \\
\text { organic, } \\
\text { suspended } \\
\text { (mg/L, } \\
\text { as C) }\end{array}$ & $\begin{array}{c}\text { Local } \\
\text { well } \\
\text { no. }\end{array}$ \\
\hline 31 & 51 & 7.3 & 0.40 & 28 & 141 & 20 & 13,000 & 160 & 2.4 & 0.8 & EEA-S1 \\
\hline 5 & 12 & 3.7 & $<.10$ & 13 & 44 & 100 & 12 & 10 & .6 & .2 & EEA-S2 \\
\hline 489 & 130 & 2,100 & .10 & 16 & 4,510 & 10 & 40,000 & 550 & 14 & .6 & EEA-S3 \\
\hline 11 & 30 & 4.6 & $<10$ & 20 & 198 & $<10$ & 28 & 34 & 3.0 & 2.2 & EEA-S4 \\
\hline 4 & 31 & 16 & .50 & 15 & 92 & 530 & 280 & 35 & 1.4 & .3 & EEA-S5 \\
\hline 266 & 16 & 4.2 & .10 & 10 & 334 & 10 & 230 & 100 & 1.3 & $>3.3$ & EEA-S6 \\
\hline 15 & 54 & 20 & .20 & 39 & 175 & 30 & 150 & 23 & 2.7 & .9 & EEA-S7 \\
\hline 5 & 40 & 9.5 & $<.10$ & 13 & 96 & 150 & 470 & 82 & 1.1 & .2 & EEA-s8 \\
\hline 5 & 40 & 9.3 & $<.10$ & 14 & 95 & 150 & 350 & 81 & 1.0 & .2 & EEA-S8 \\
\hline 105 & 31 & 3.1 & .10 & 32 & 204 & 20 & 150 & 47 & 2.2 & .9 & EEA-S9 \\
\hline 278 & 81 & 4.1 & 1.0 & 46 & 442 & $<10$ & 170 & 72 & 3.5 & .1 & EEA-S10 \\
\hline 29 & .60 & 3.0 & .70 & 40 & 89 & 30 & 9,600 & 110 & .7 & -. & EEA-S11 \\
\hline 16 & 8.4 & 2.9 & $<.10$ & 11 & 51 & 20 & 3,500 & 85 & 1.3 & 1.1 & EEA-S12 \\
\hline 29 & 15 & 6.1 & .10 & 18 & 51 & 20 & 1,500 & 59 & 1.0 & .1 & EEA-S13 \\
\hline 6 & 32 & 3.4 & .50 & 9.9 & 68 & 550 & 9 & 27 & 1.0 & .7 & EEA-S14 \\
\hline 6 & 41 & 27 & 1.5 & 41 & 150 & 1,700 & 970 & 89 & 1.9 & 1.3 & EEA-S15 \\
\hline 4 & 16 & 6.9 & $<.10$ & 24 & 72 & 60 & 19 & 22 & 1.4 & .4 & EEA-S16 \\
\hline 4 & 11 & 2.0 & $<.10$ & 18 & 1,010 & 10 & 19 & 22 & 3.8 & 2.3 & EEA-S17 \\
\hline 75 & 11 & 3.0 & .10 & 20 & 116 & $<10$ & 28 & 34 & 1.2 & .9 & EEA-S18 \\
\hline 315 & 1.0 & 2.1 & $<.10$ & 21 & 342 & 20 & 12,000 & 730 & 14 & 5.0 & EEA-S19 \\
\hline 6 & 6.9 & 1.9 & $<.10$ & 16 & 37 & -- & 350 & 110 & 1.3 & 1.1 & EEA-S20 \\
\hline 146 & 1.7 & 3.6 & .60 & 42 & 210 & $<10$ & 1,900 & 58 & 3.9 & .1 & EEA-M1 \\
\hline 252 & 7.9 & 2.3 & 1.3 & 17 & 304 & 40 & 41 & 3 & 7.7 & .3 & EEA-M2 \\
\hline 251 & 2.5 & 20 & .50 & 38 & 353 & 10 & 2,300 & 98 & 4.5 & .3 & EEA-M3 \\
\hline 285 & 19 & 3.0 & .30 & 57 & 387 & 20 & 110 & 18 & 4.1 & .9 & EEA-M4 \\
\hline 280 & 8.7 & 4.6 & .70 & 32 & 392 & 10 & 27 & 5 & 5.4 & 3.5 & EEA-M5 \\
\hline 267 & .40 & 12 & .40 & 48 & 368 & 10 & 2,600 & 220 & 2.5 & .2 & EEA-M6 \\
\hline 267 & .50 & 11 & .40 & 48 & 374 & 20 & 2,700 & 220 & 2.7 & .3 & EEA-M6 \\
\hline 198 & 510 & 4.4 & .20 & 31 & 1,010 & 10 & 19 & 22 & 3.8 & 2.3 & EEA-M7 \\
\hline 279 & $<.10$ & 9.7 & .70 & 43 & 317 & 10 & 3,800 & 190 & - & -- & EEA-M8 \\
\hline
\end{tabular}




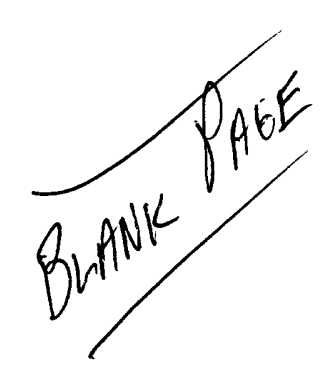




\section{ILLUSTRATIONS}




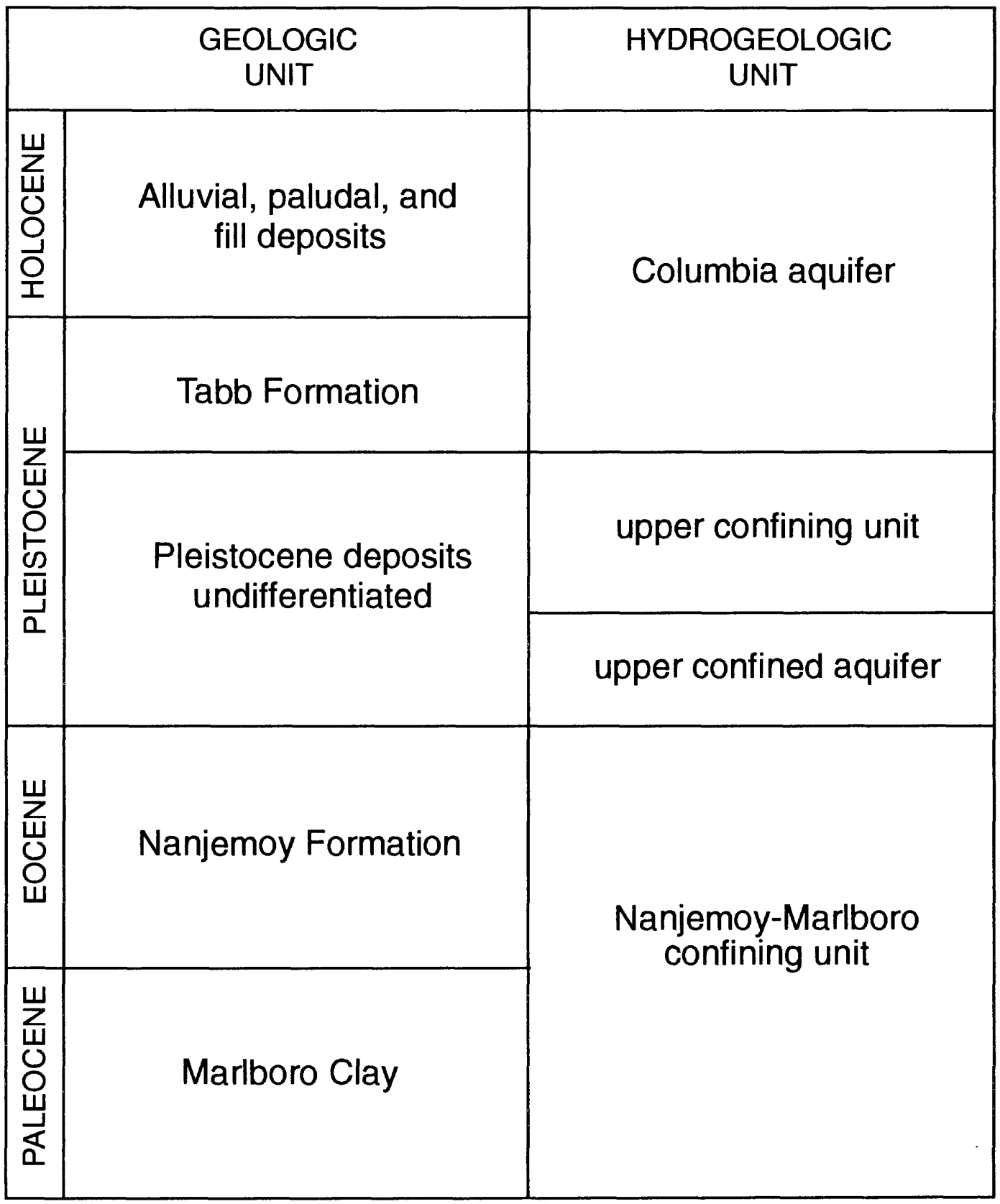

NOT TO SCALE

Figure 2. Geologic and hydrogeologic units of the shallow aquifer system at the Explosive Experimental Area, Naval Surface Warfare Center, Dahlgren Site, Dalhgren, Virginia. From unpublished data on file in the Virginia District office of the U.S. Geological Survey. 


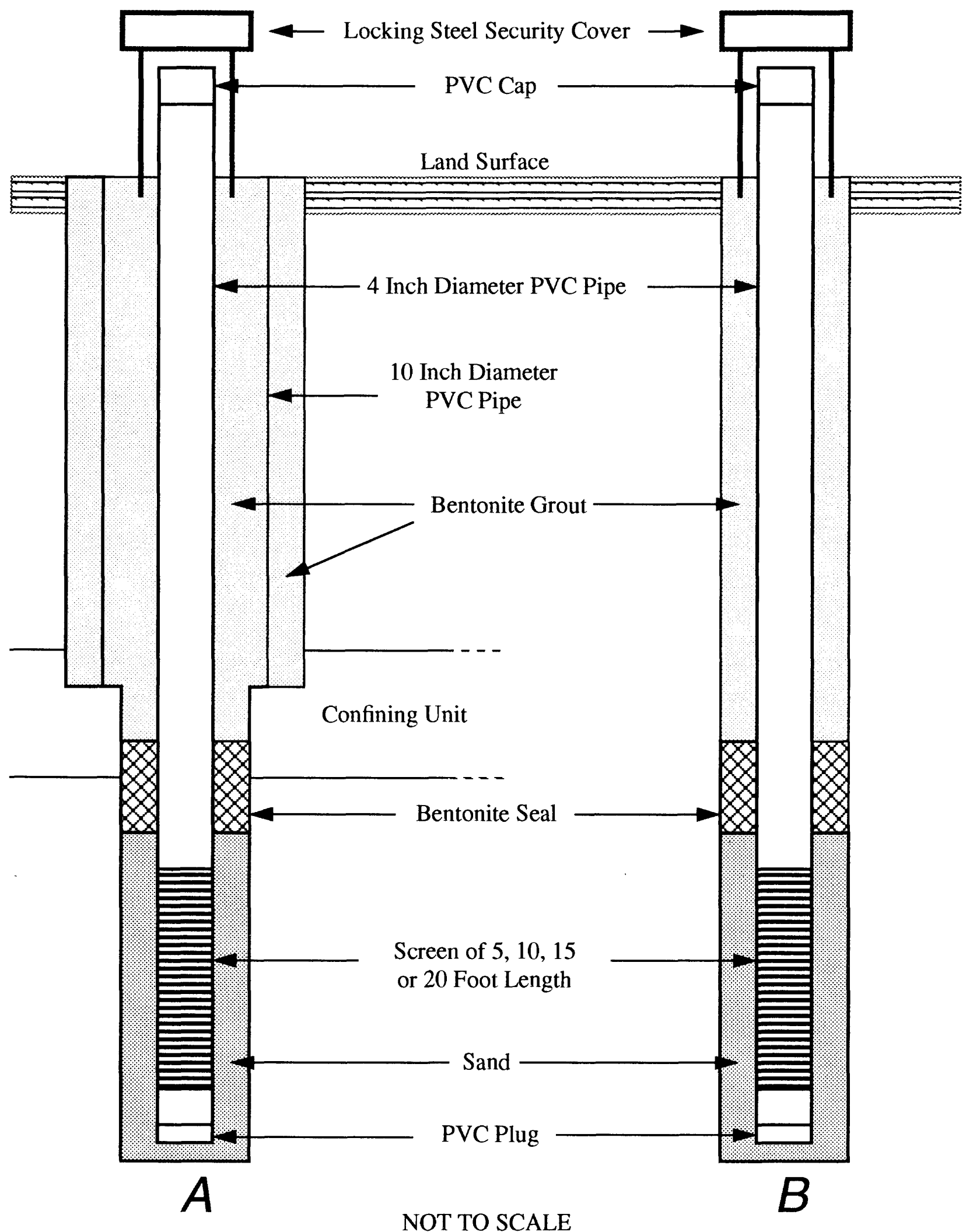

Figure 3. Standard construction of wells completed in a $(A)$ confined aquifer and $(B)$ unconfined aquifer. 

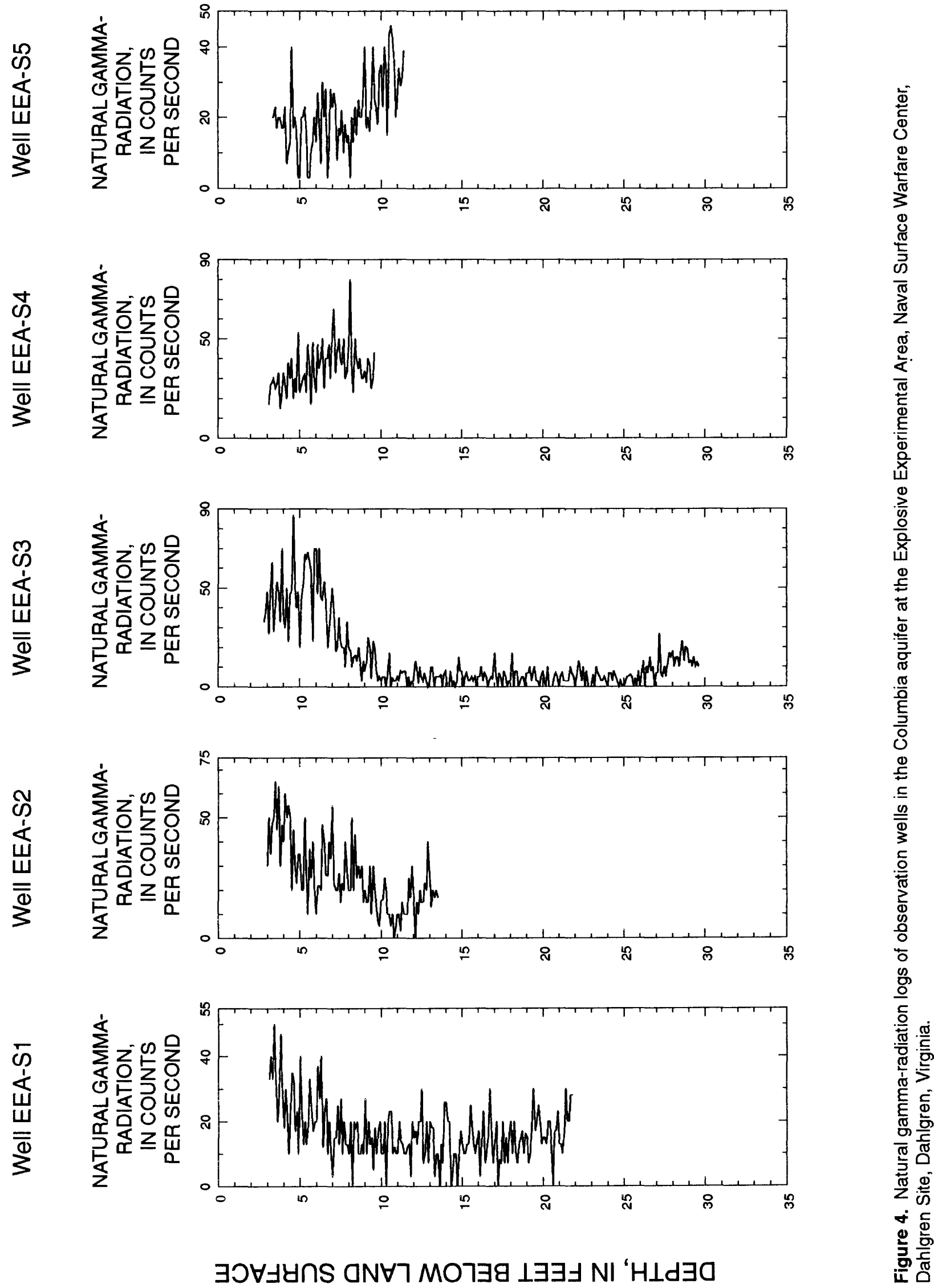

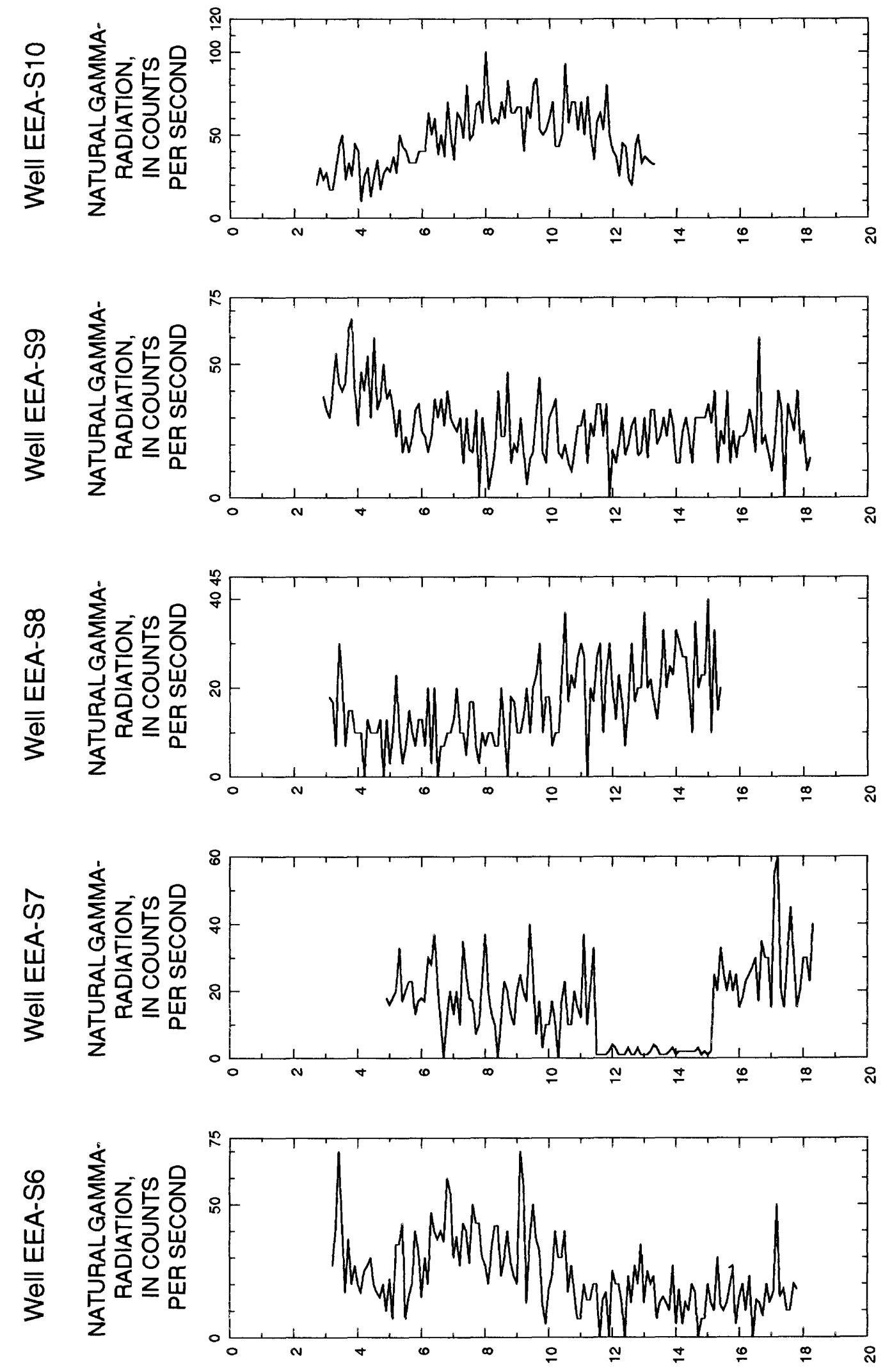

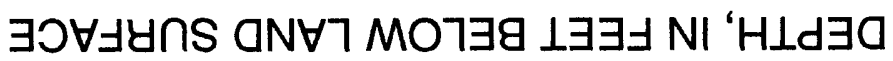

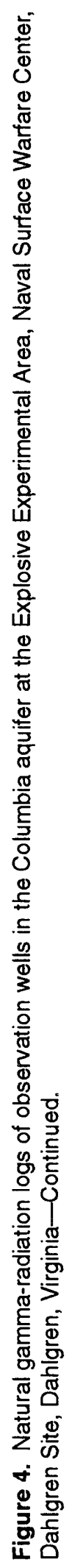



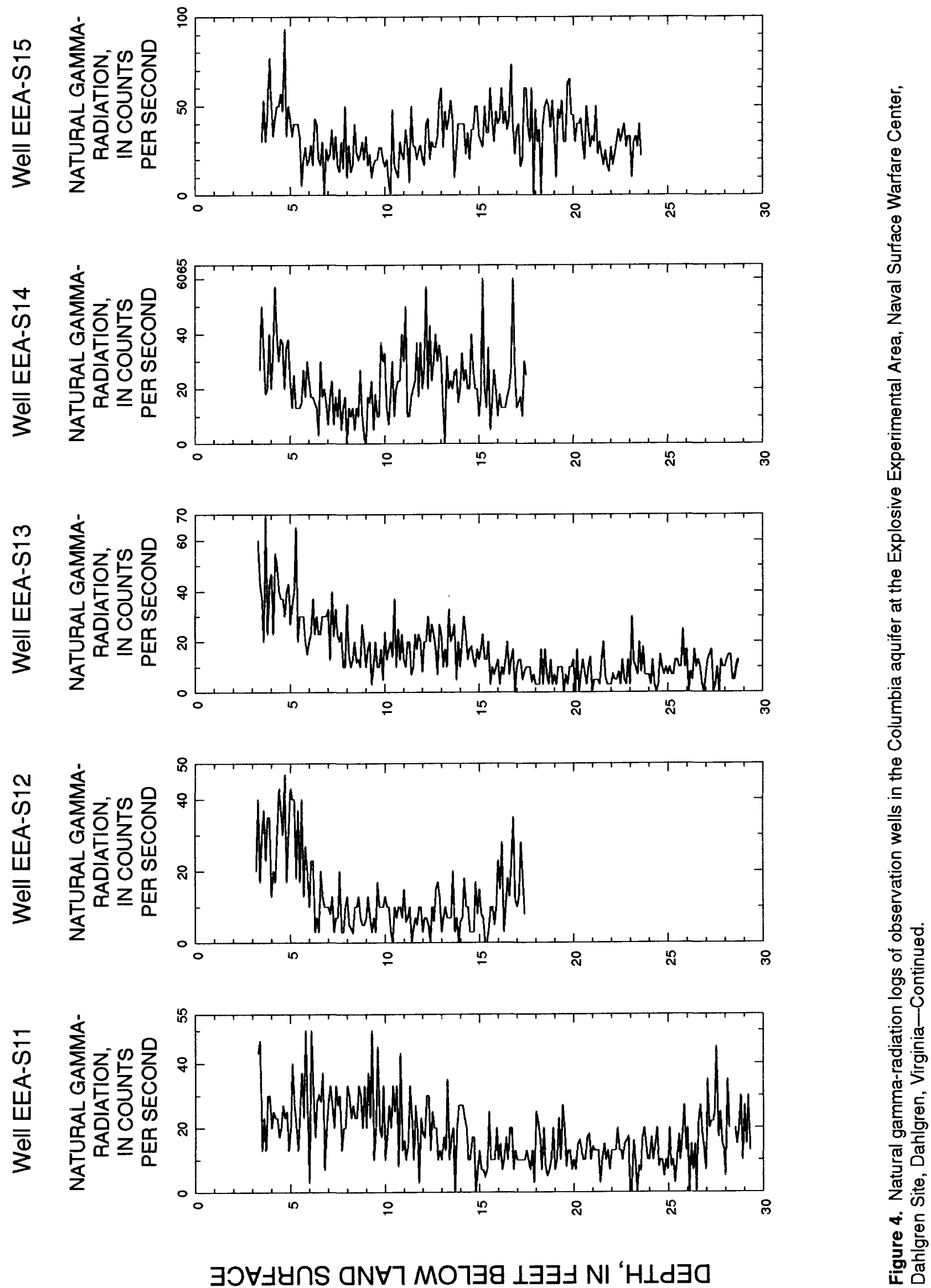

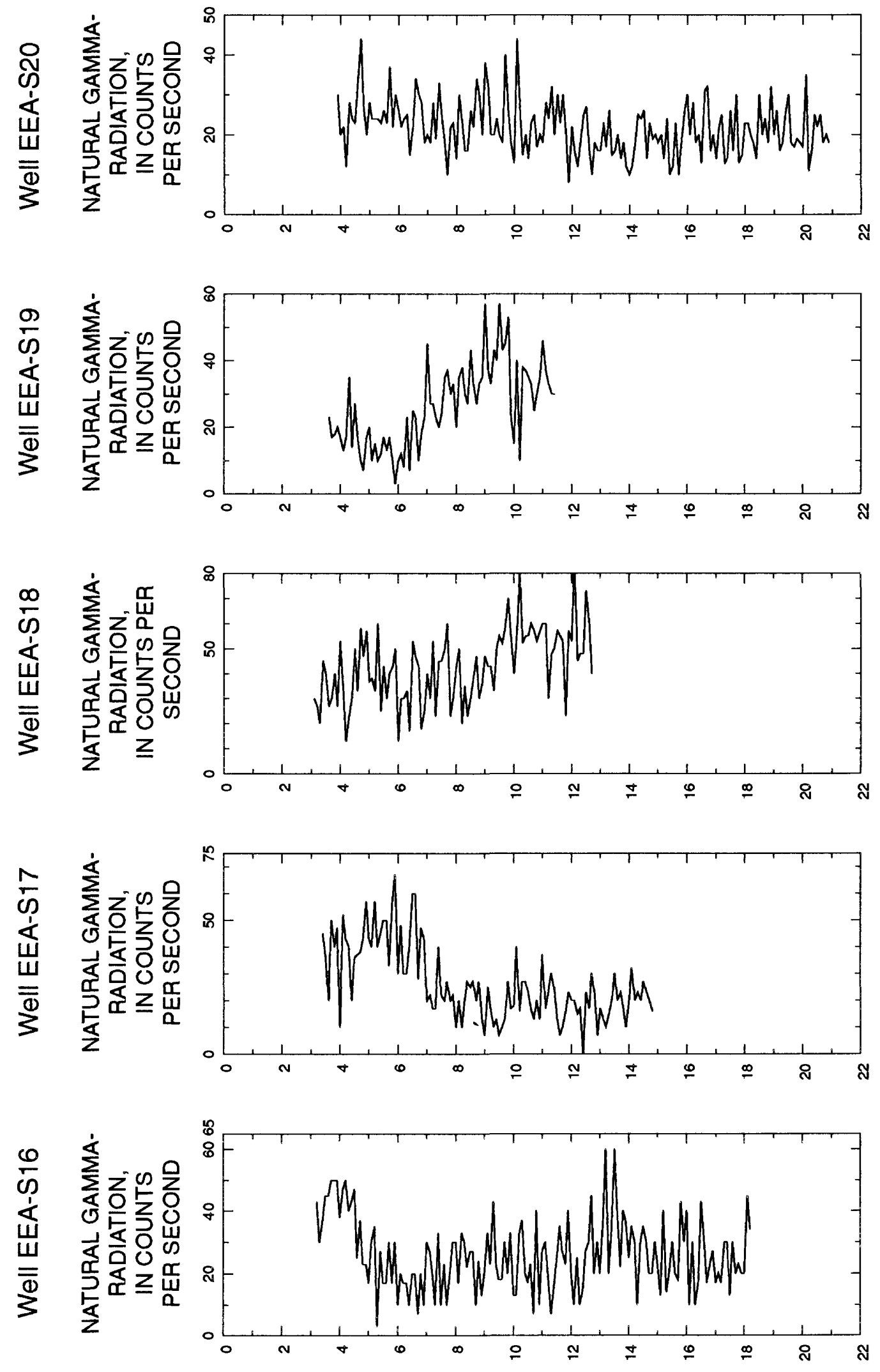

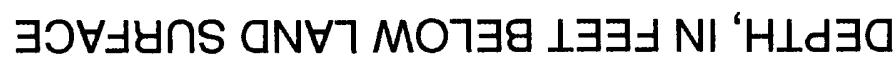



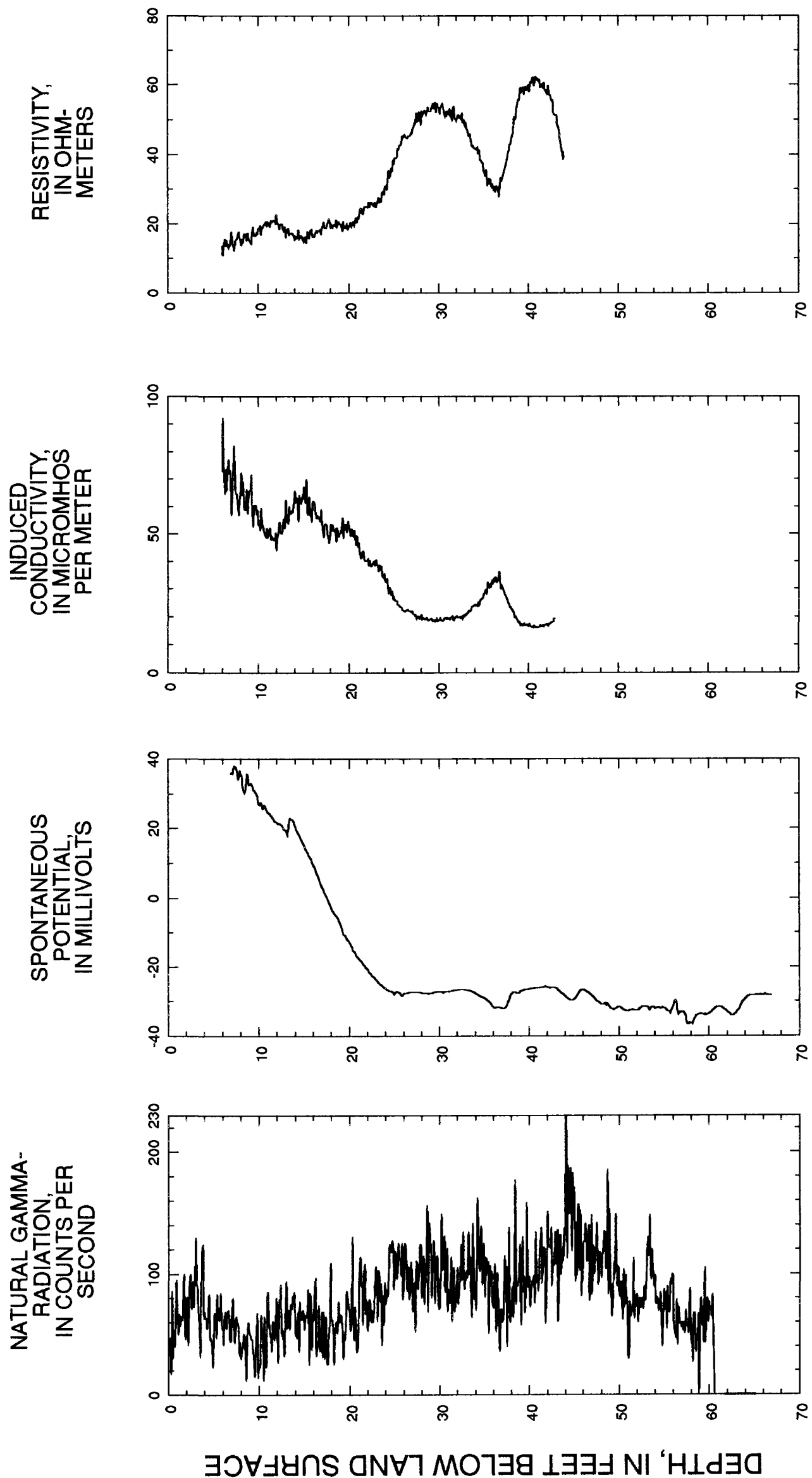

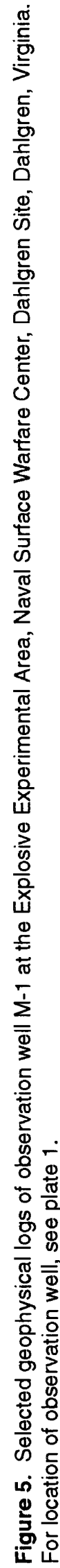



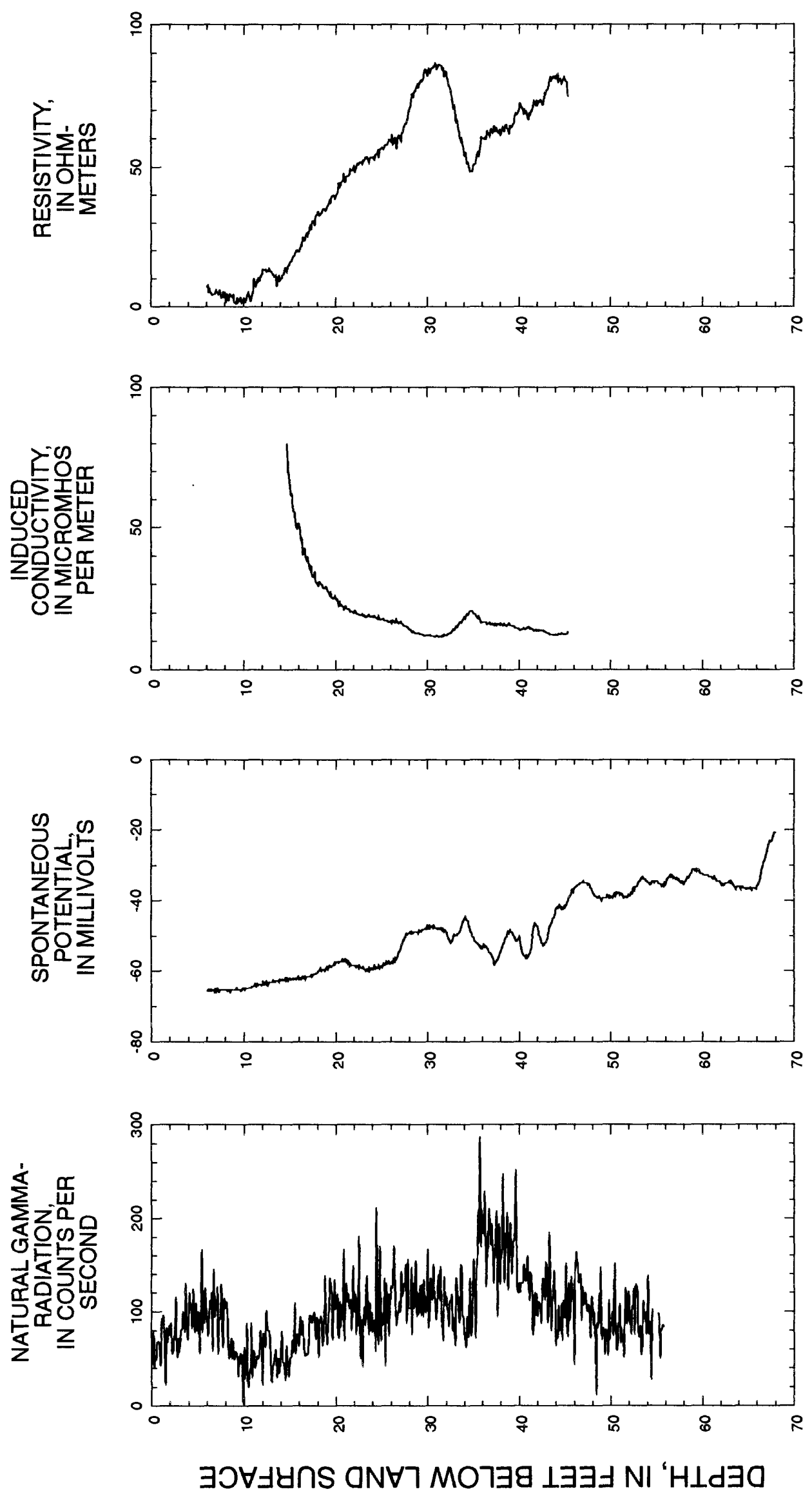

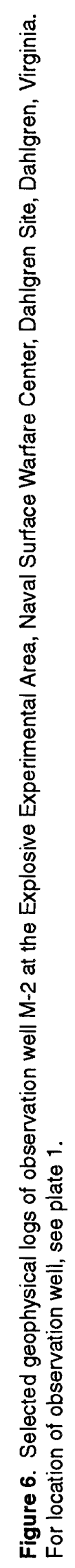



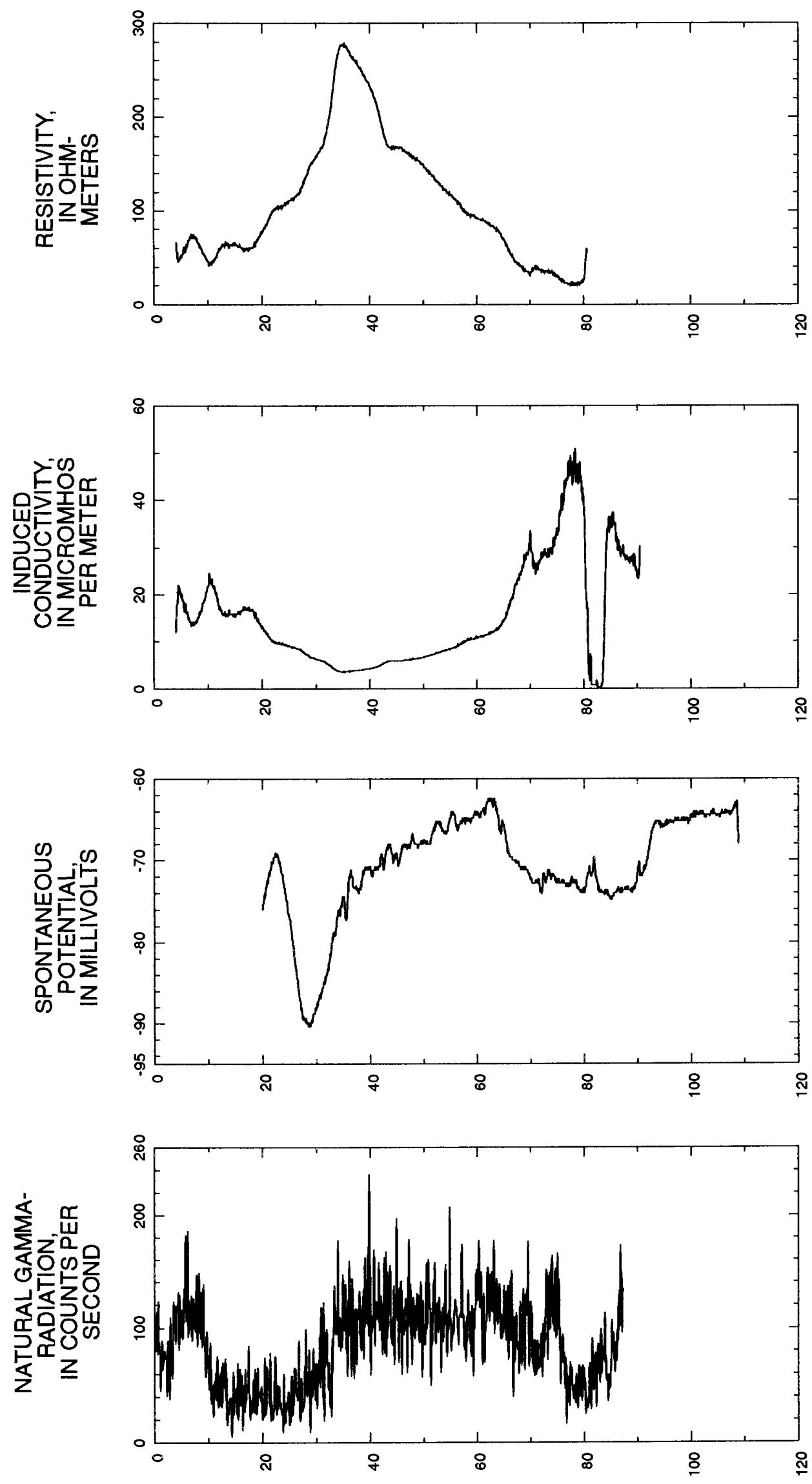

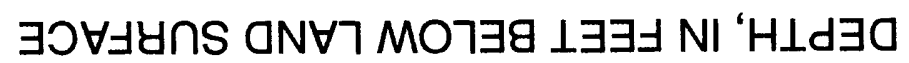



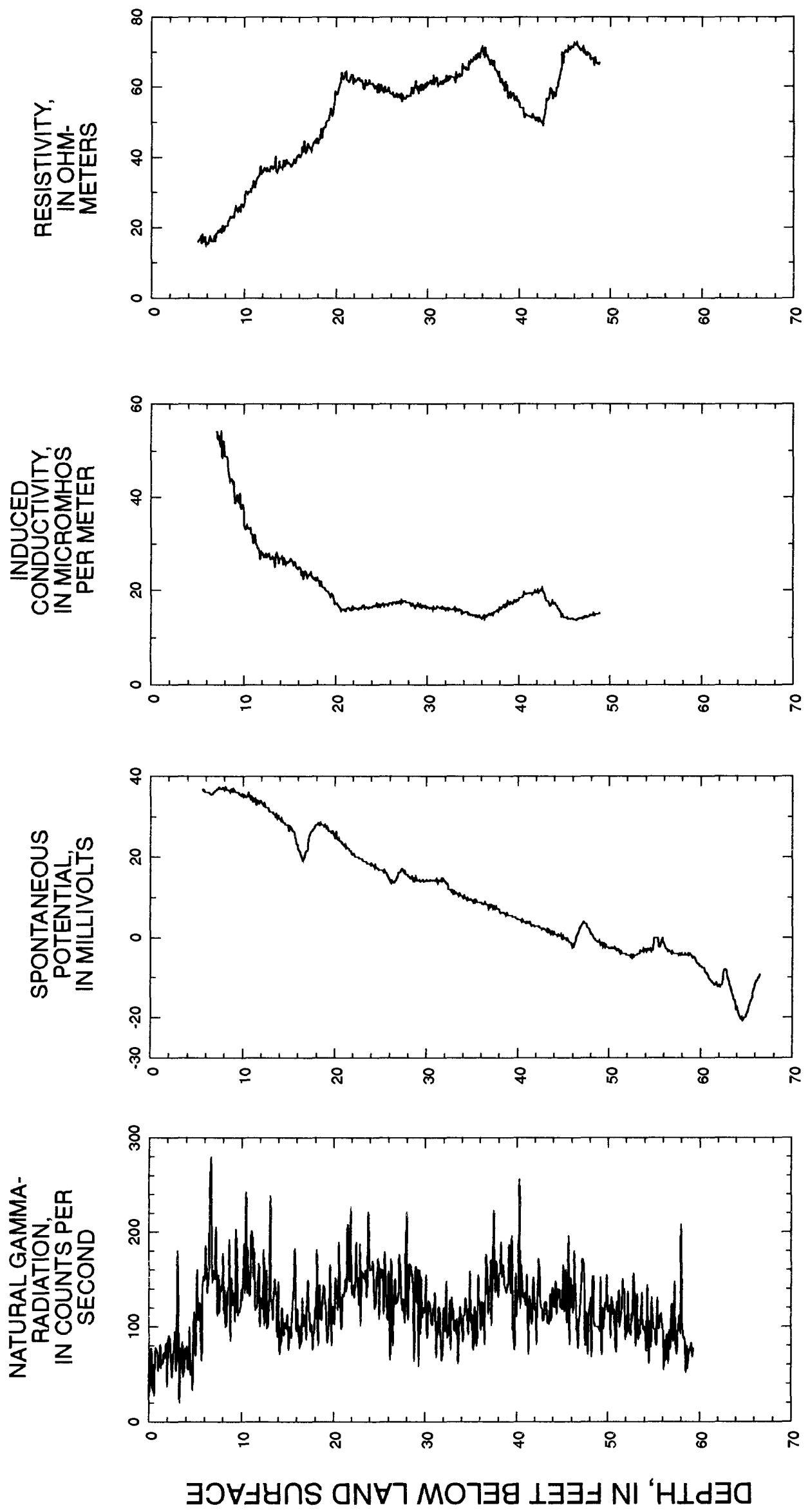

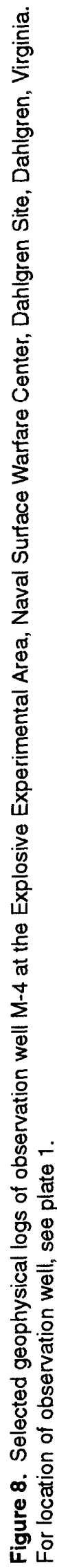



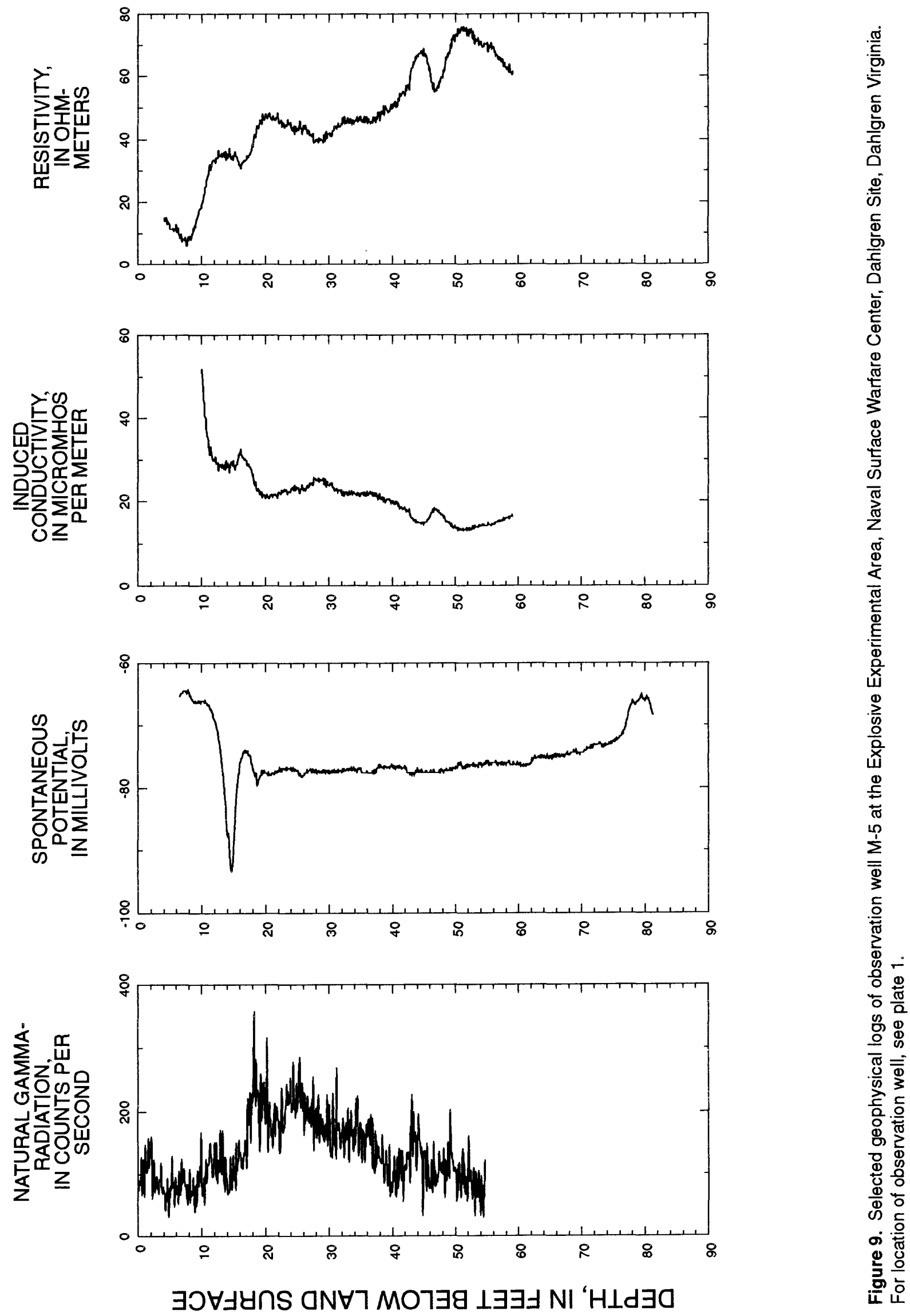


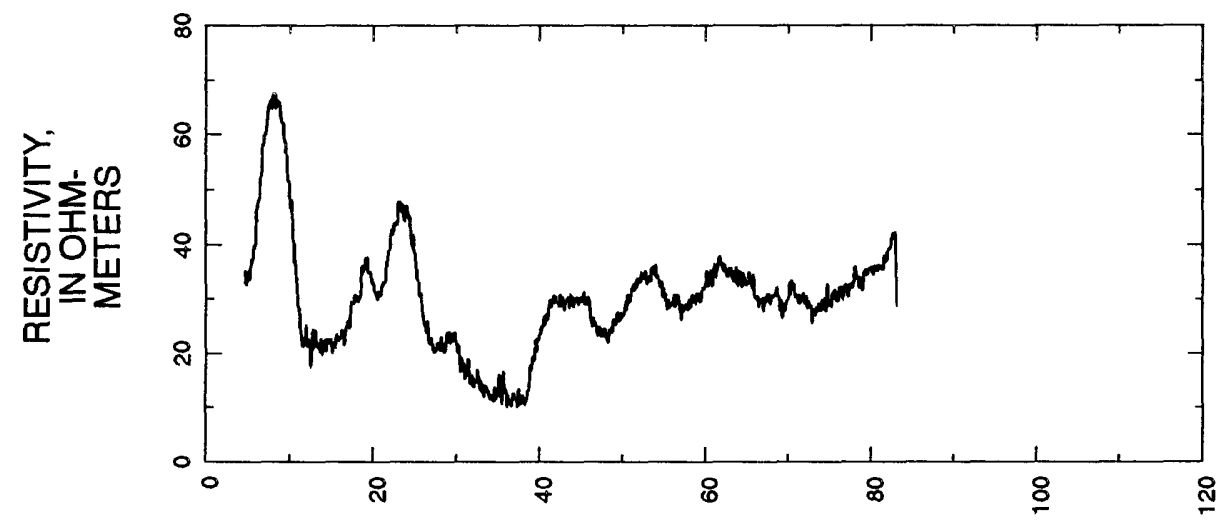

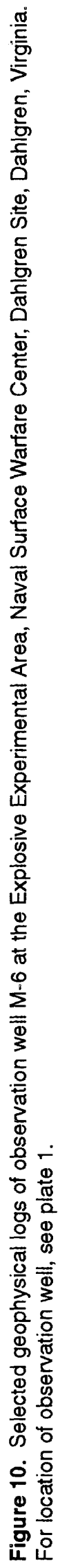

Figures 47 

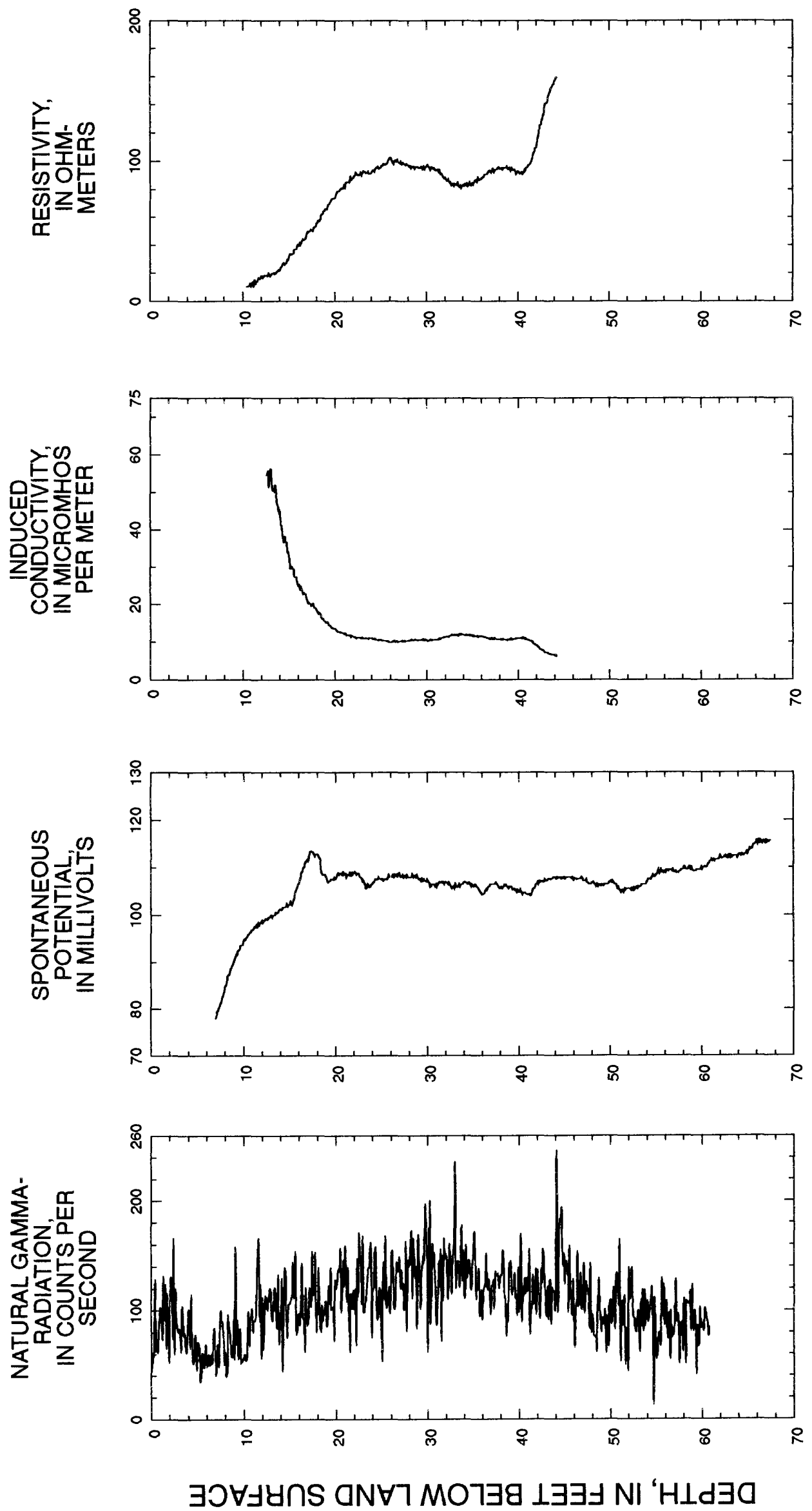

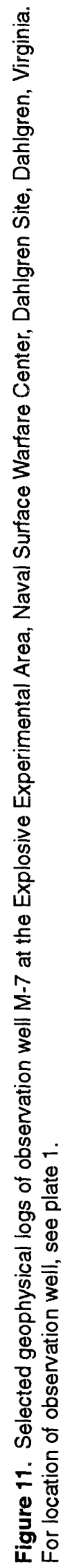




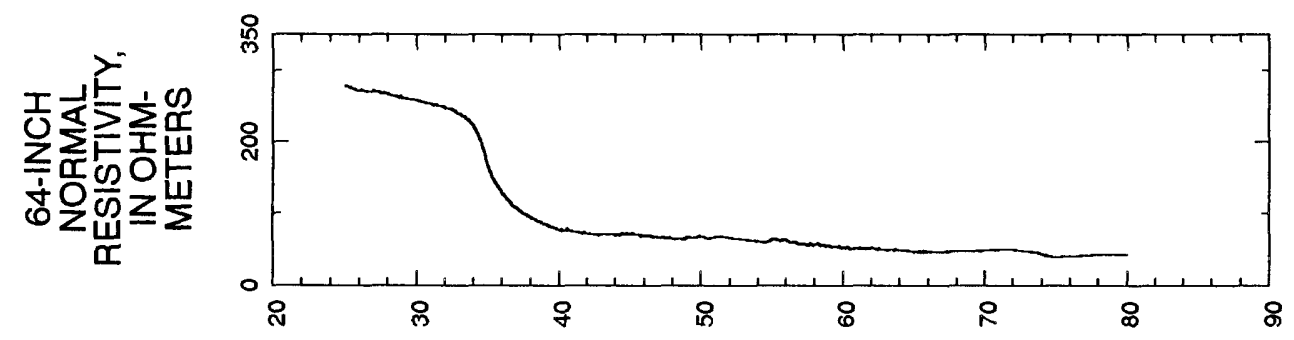

政
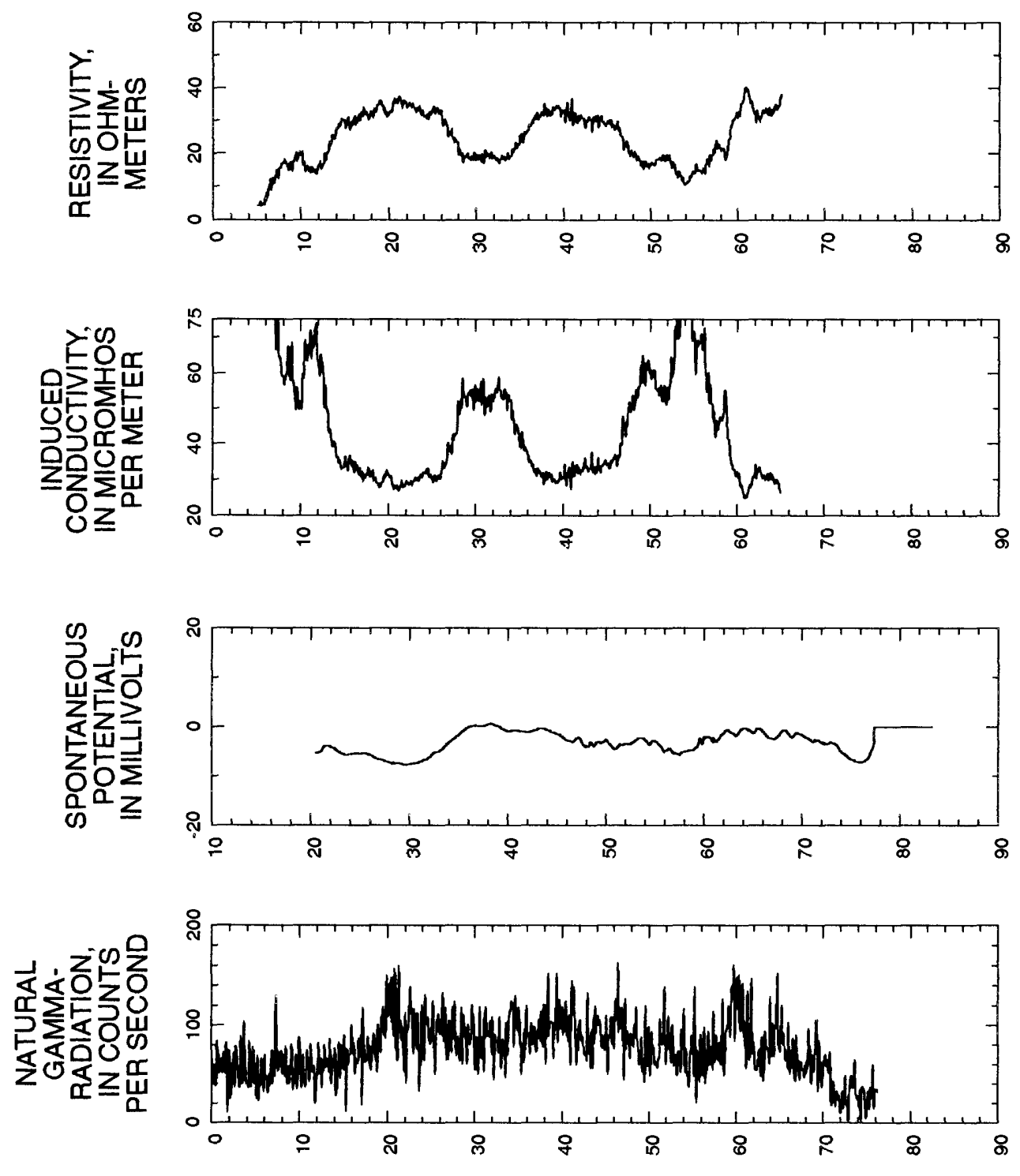

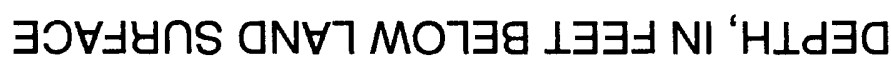




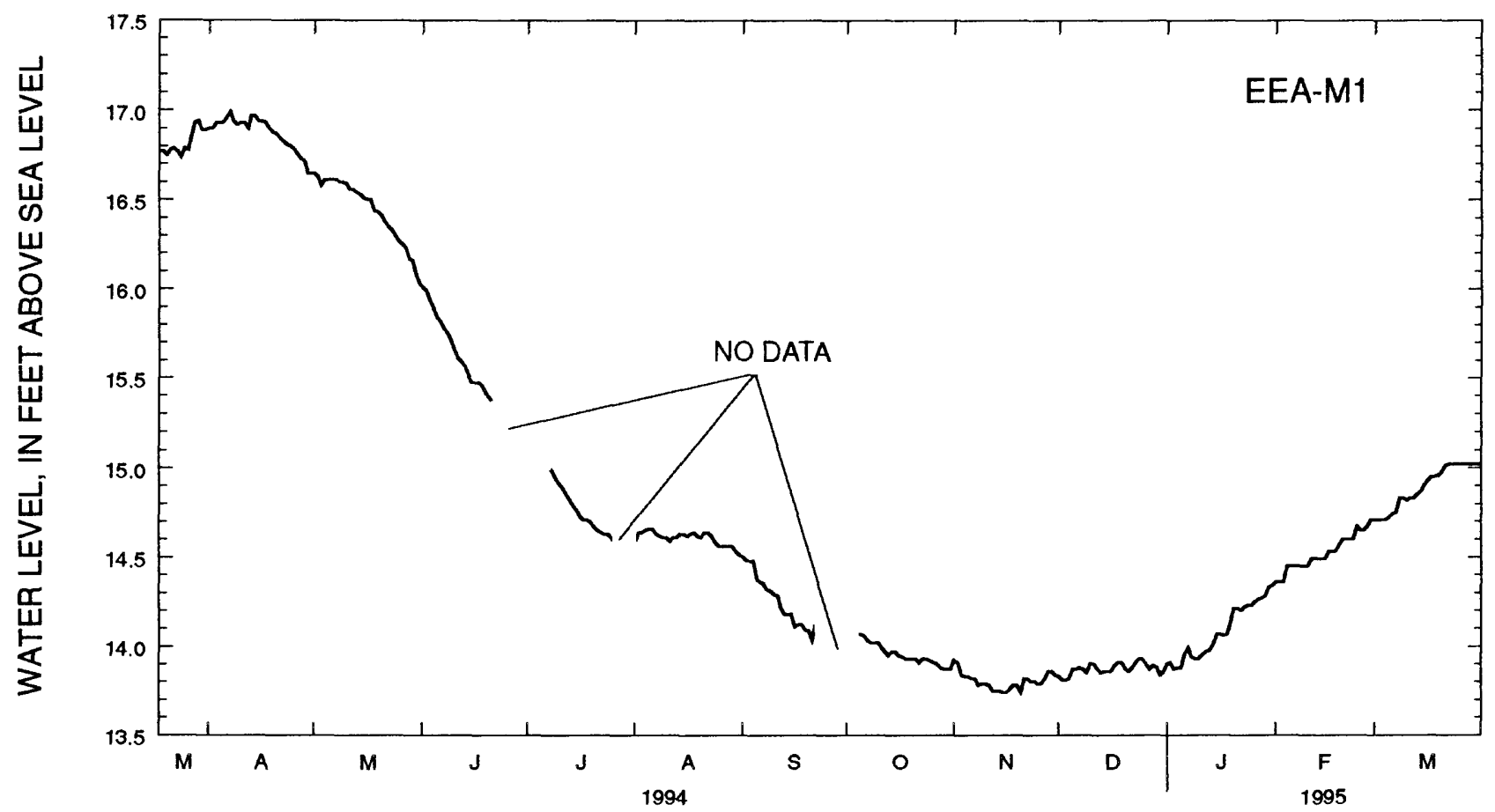

Figure 13. Hydrograph showing minimum daily water level relative to sea level for observation well EEA-M1 at the Explosive Experimental Area, Naval Surface Warfare Center, Dahlgren Site, Dahlgren, Virginia. For location of observation well, see plate 1.

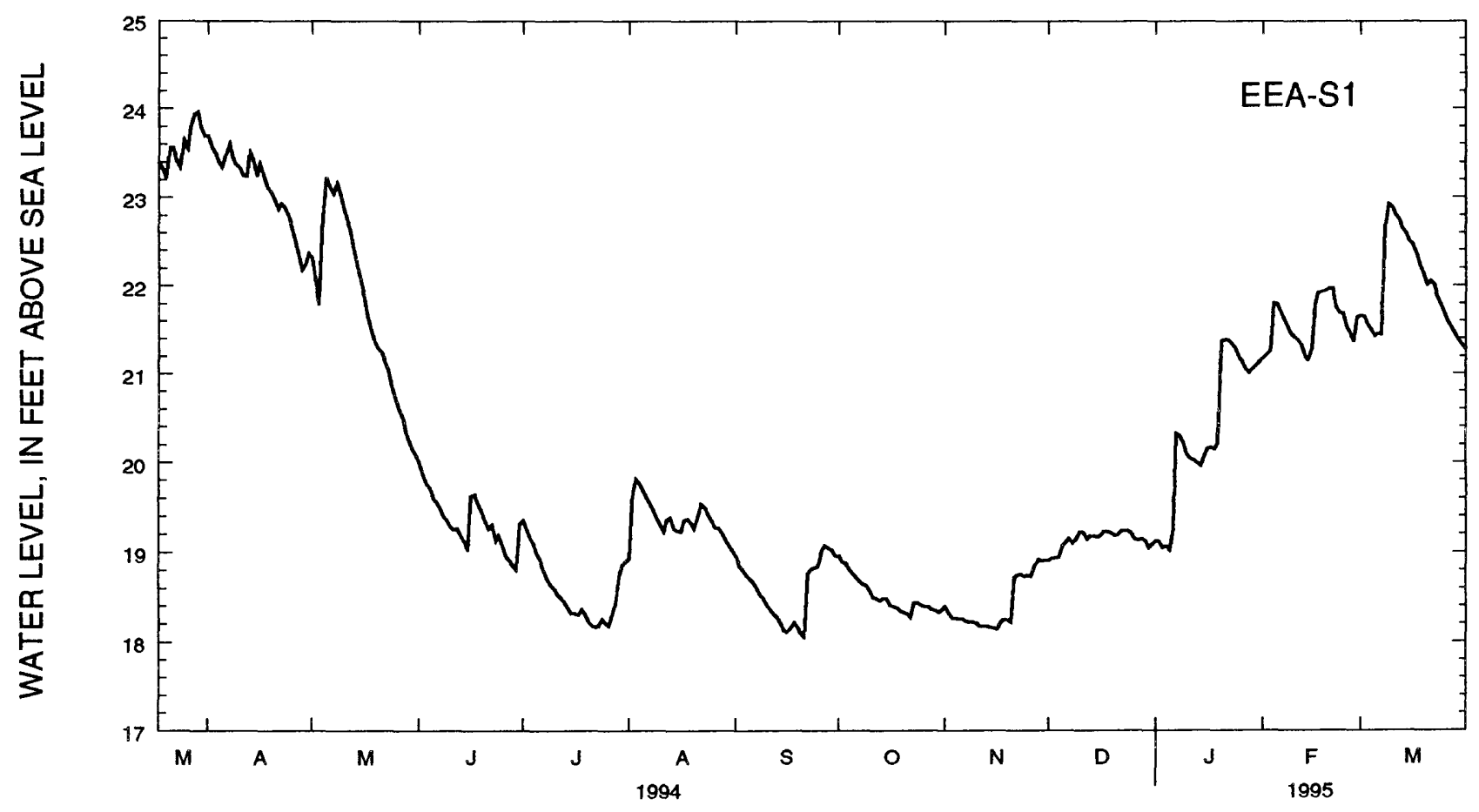

Figure 14. Hydrograph showing minimum daily water level relative to sea level for observation well EEA-S1 at the Explosive Experimental Area, Naval Surface Warfare Center, Dahlgren Site, Dahlgren, Virginia. For location of observation well, see plate 1. 


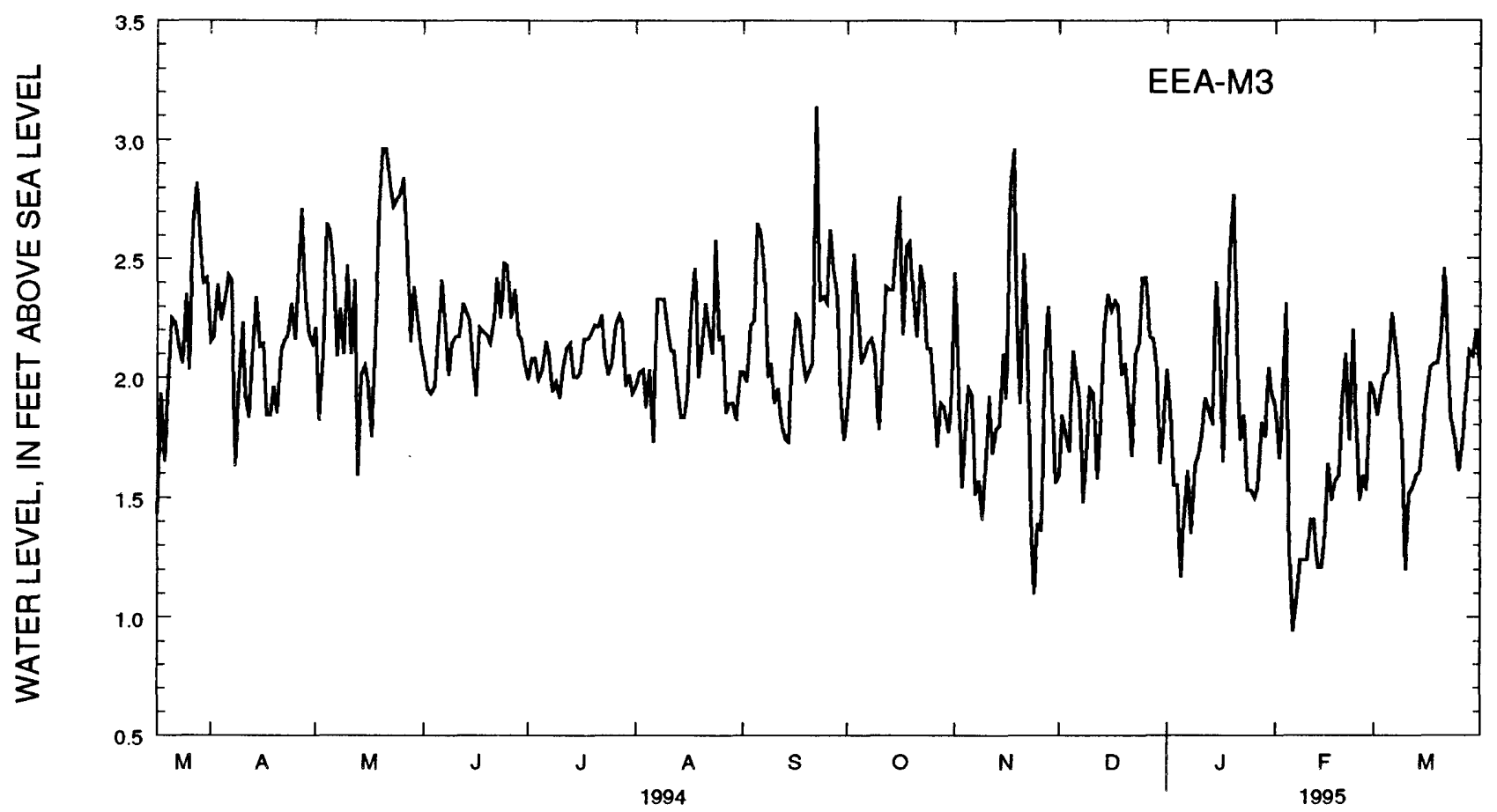

Figure 15. Hydrograph showing minimum daily water level relative to sea level for observation well EEA-M3 at the Explosive Experimental Area, Naval Surface Warfare Center, Dahlgren Site, Dahlgren, Virginia. For location of observation well, see plate 1.

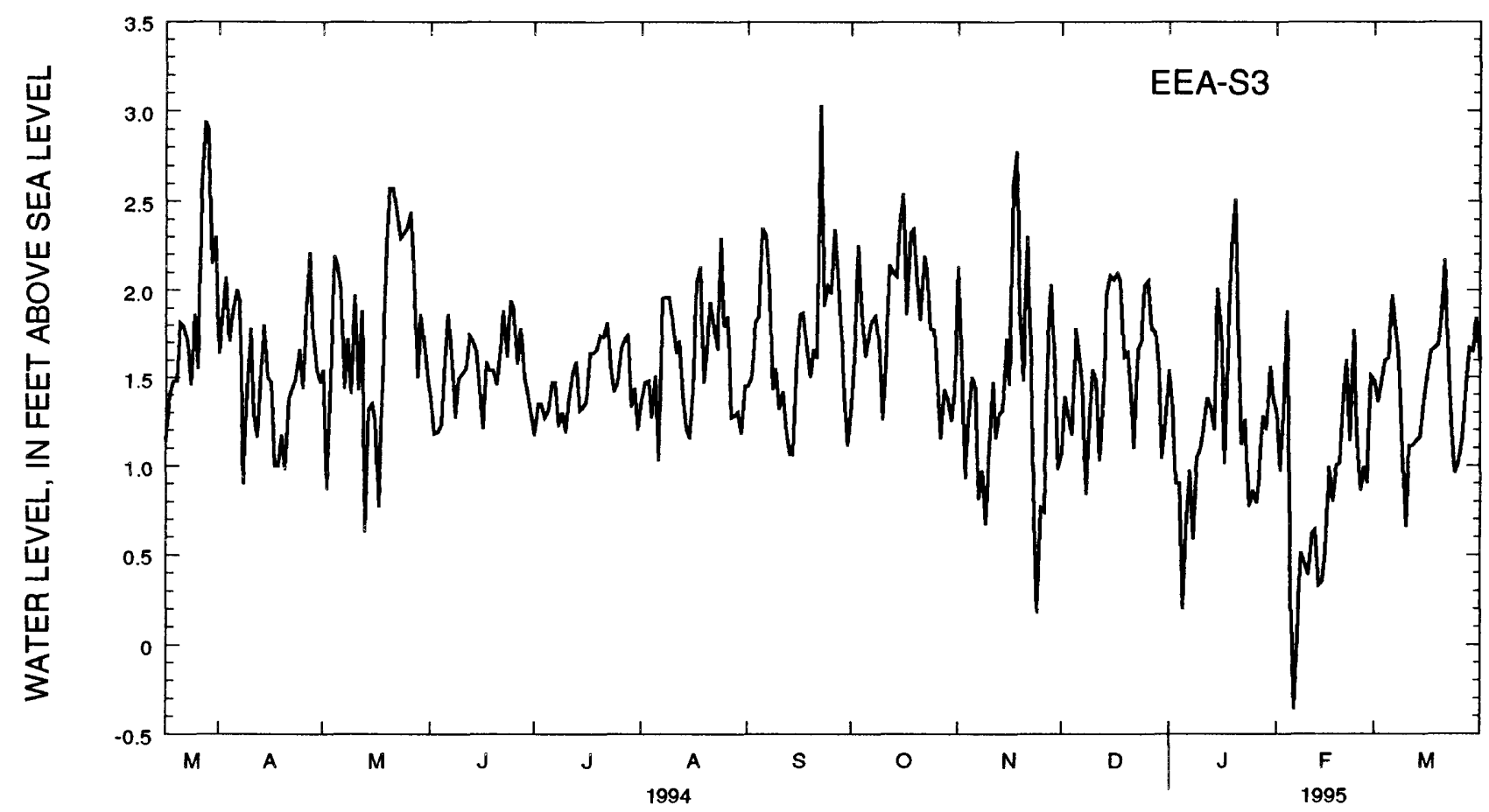

Figure 16. Hydrograph showing minimum daily water level relative to sea level for observation well EEA-S3 at the Explosive Experimental Area, Naval Surface Warfare Center, Dahlgren Site, Dahlgren, Virginia. For location of observation well, see plate 1. 


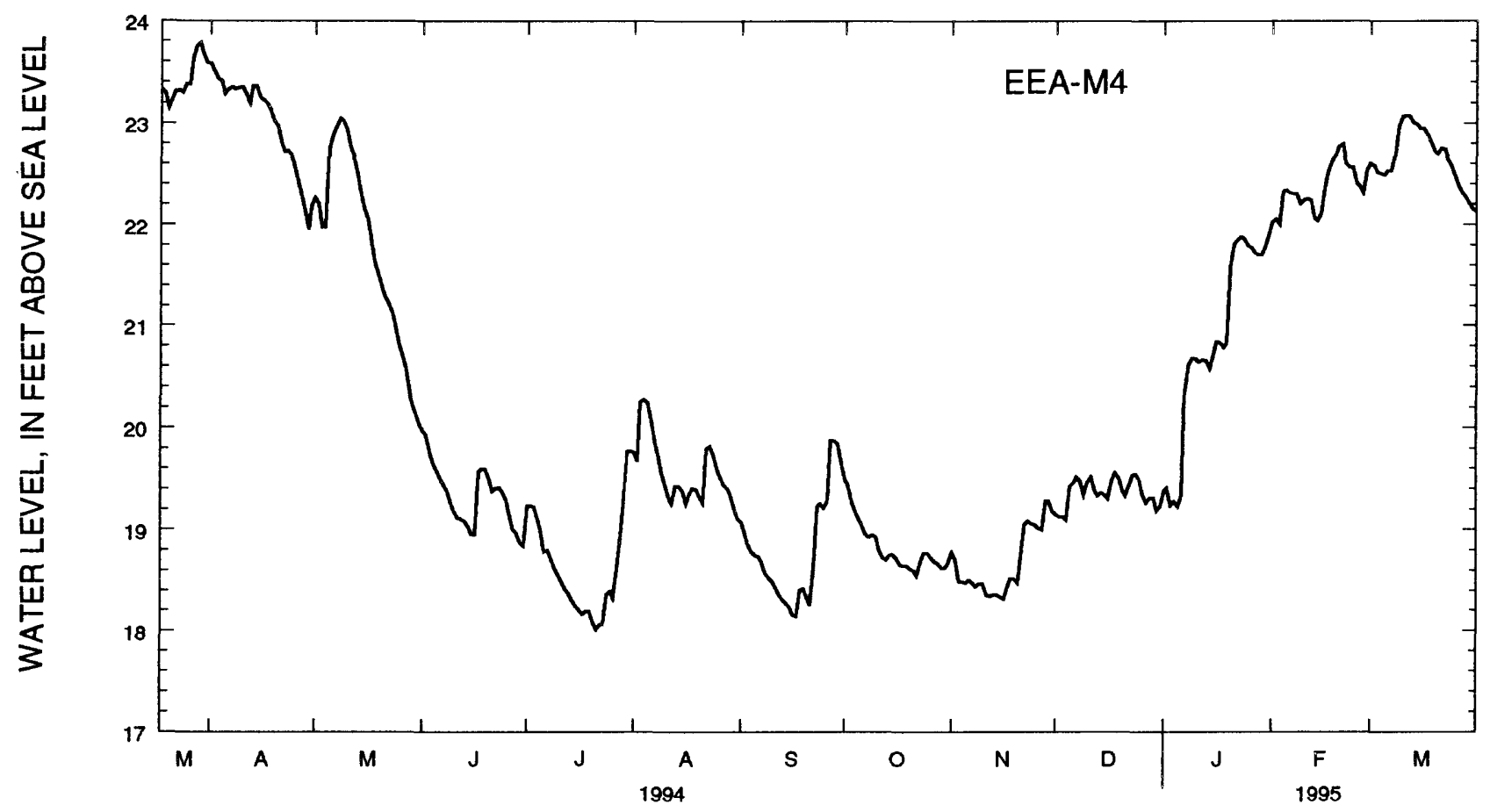

Figure 17. Hydrograph showing minimum daily water level relative to sea level for observation well EEA-M4 at the Explosive Experimental Area, Naval Surface Warfare Center, Dahlgren Site, Dahlgren, Virginia. For location of observation well, see plate 1.

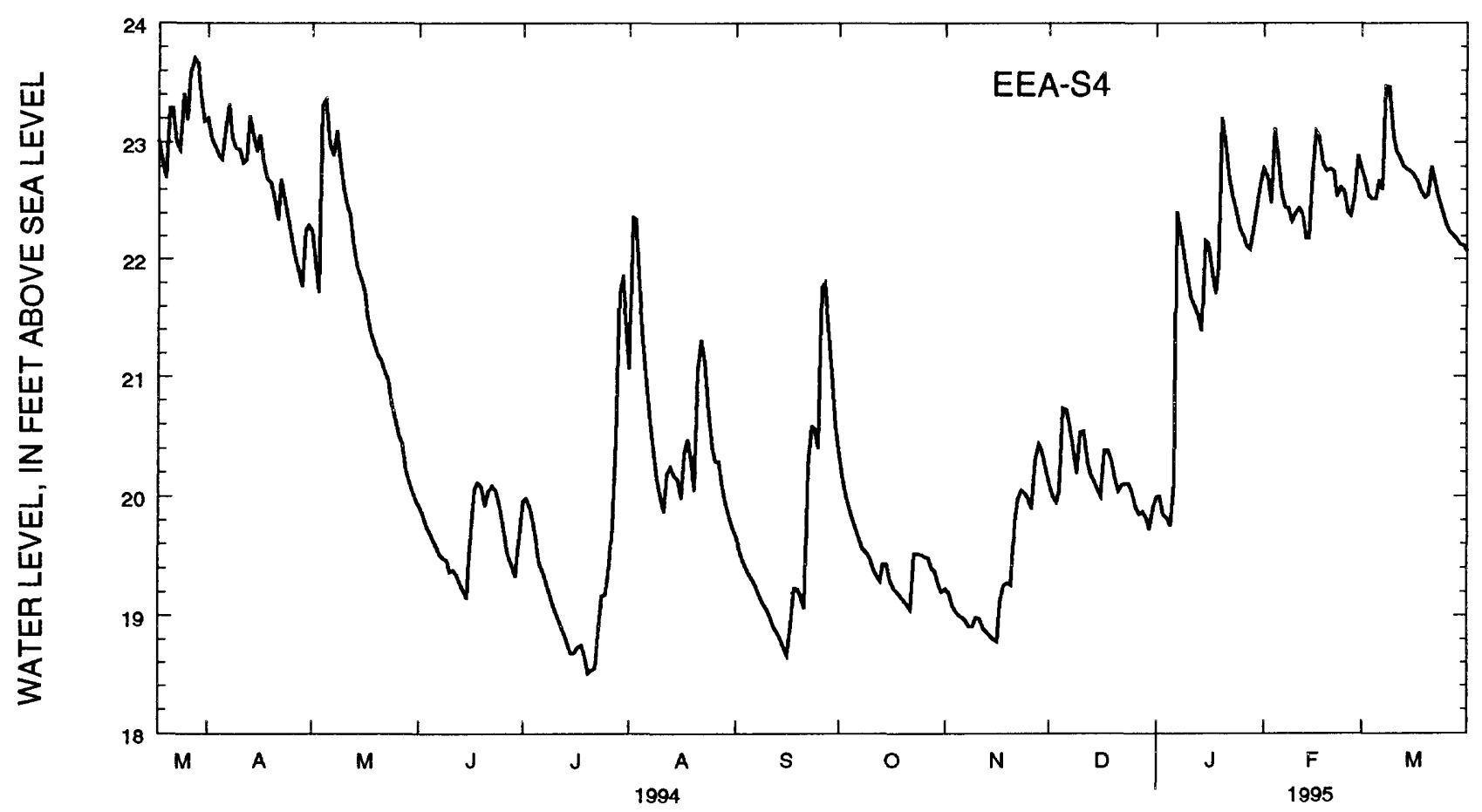

Figure 18. Hydrograph showing minimum daily water level relative to sea level for observation well EEA-S4 at the Explosive Experimental Area, Naval Surface Warfare Center, Dahlgren Site, Dahlgren, Virginia. For location of observation well, see plate 1. 


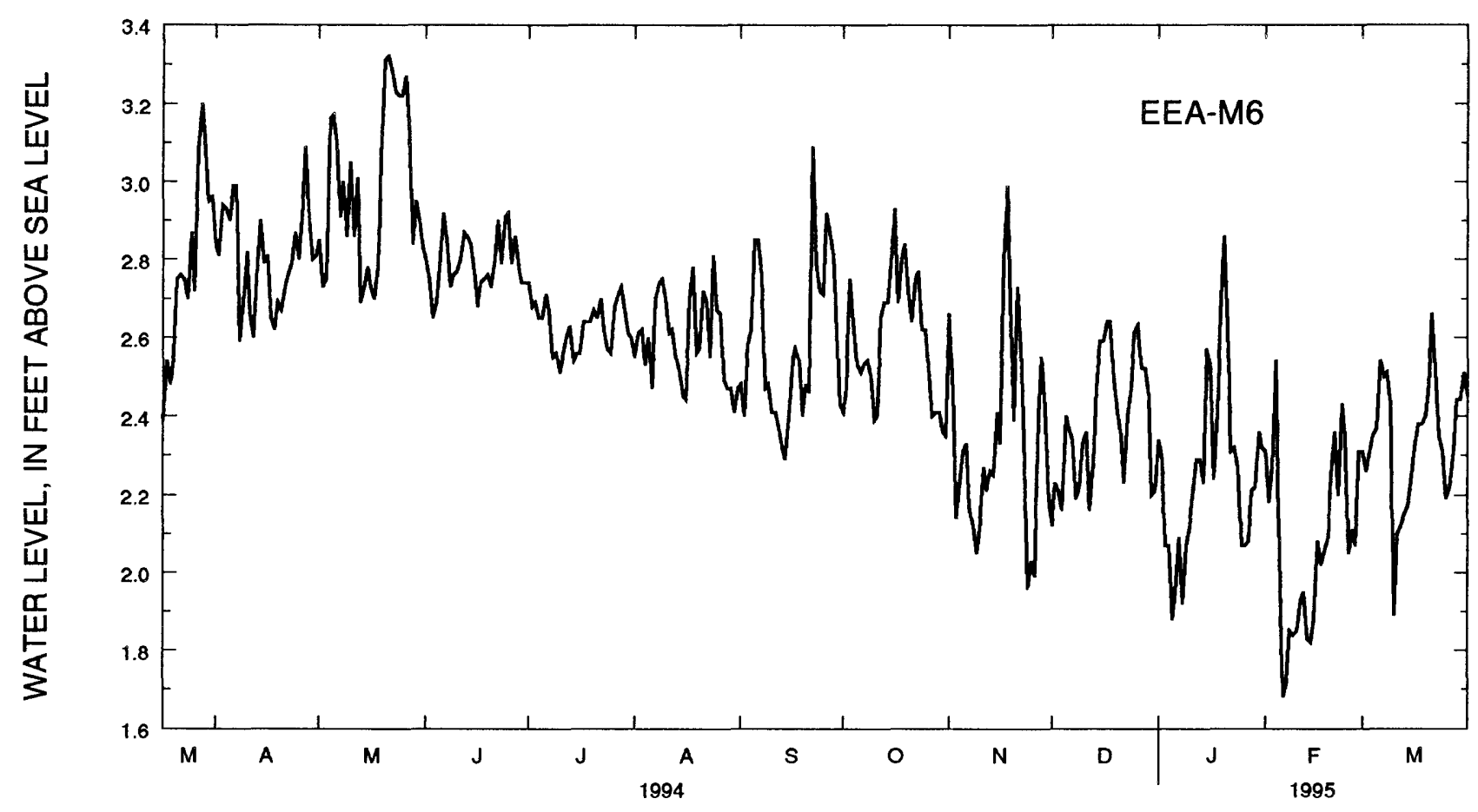

Figure 19. Hydrograph showing minimum daily water level relative to sea level for observation well EEA-M6 at the Explosive Experimental Area, Naval Surface Warfare Center, Dahlgren Site, Dahlgren, Virginia. For location of observation well, see plate 1.

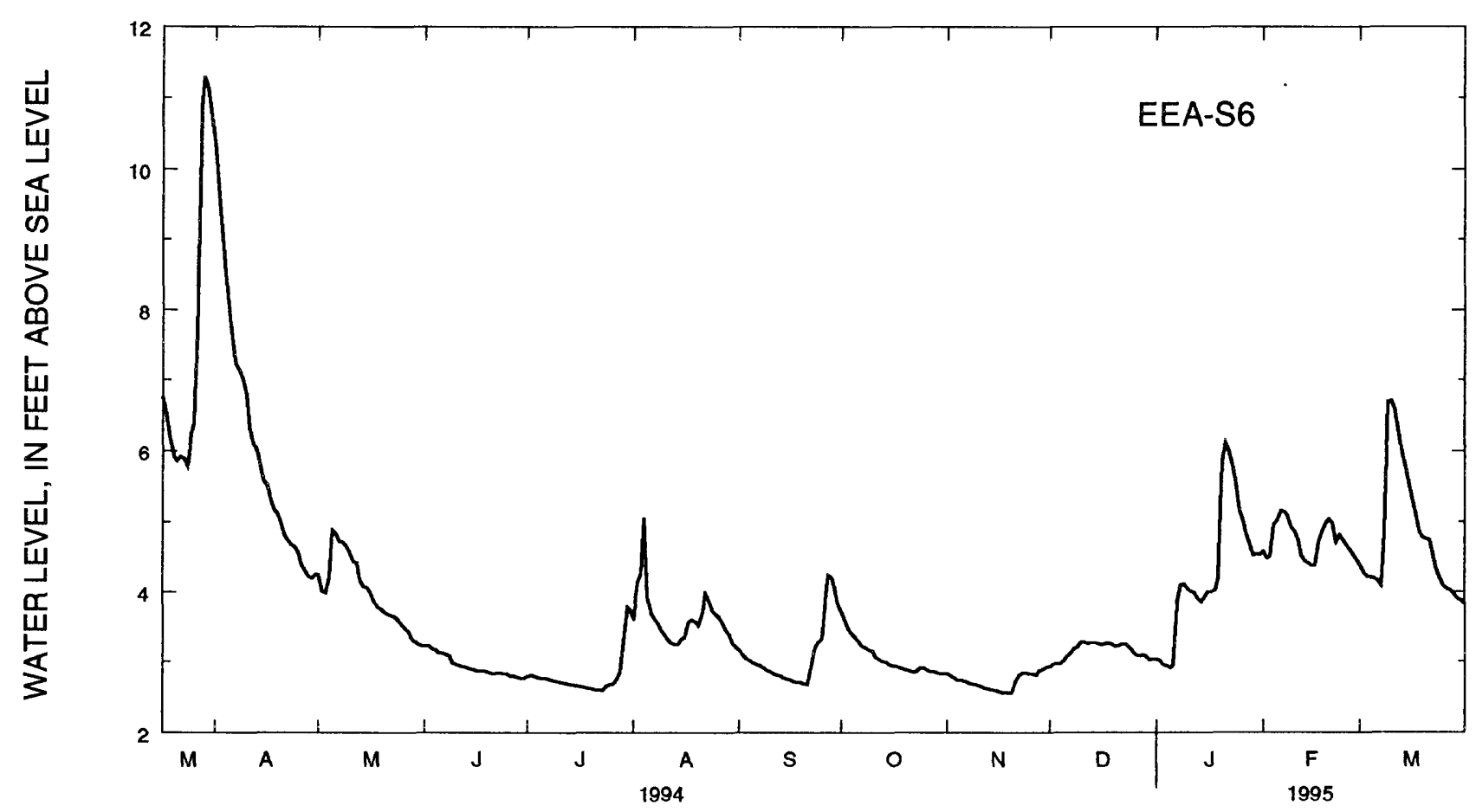

Figure 20. Hydrograph showing minimum daily water level relative to sea level for observation well EEA-S6 at the Explosive Experimental Area, Naval Surface Warfare Center, Dahlgren Site, Dahlgren, Virginia. For location of observation well, see plate 1. 


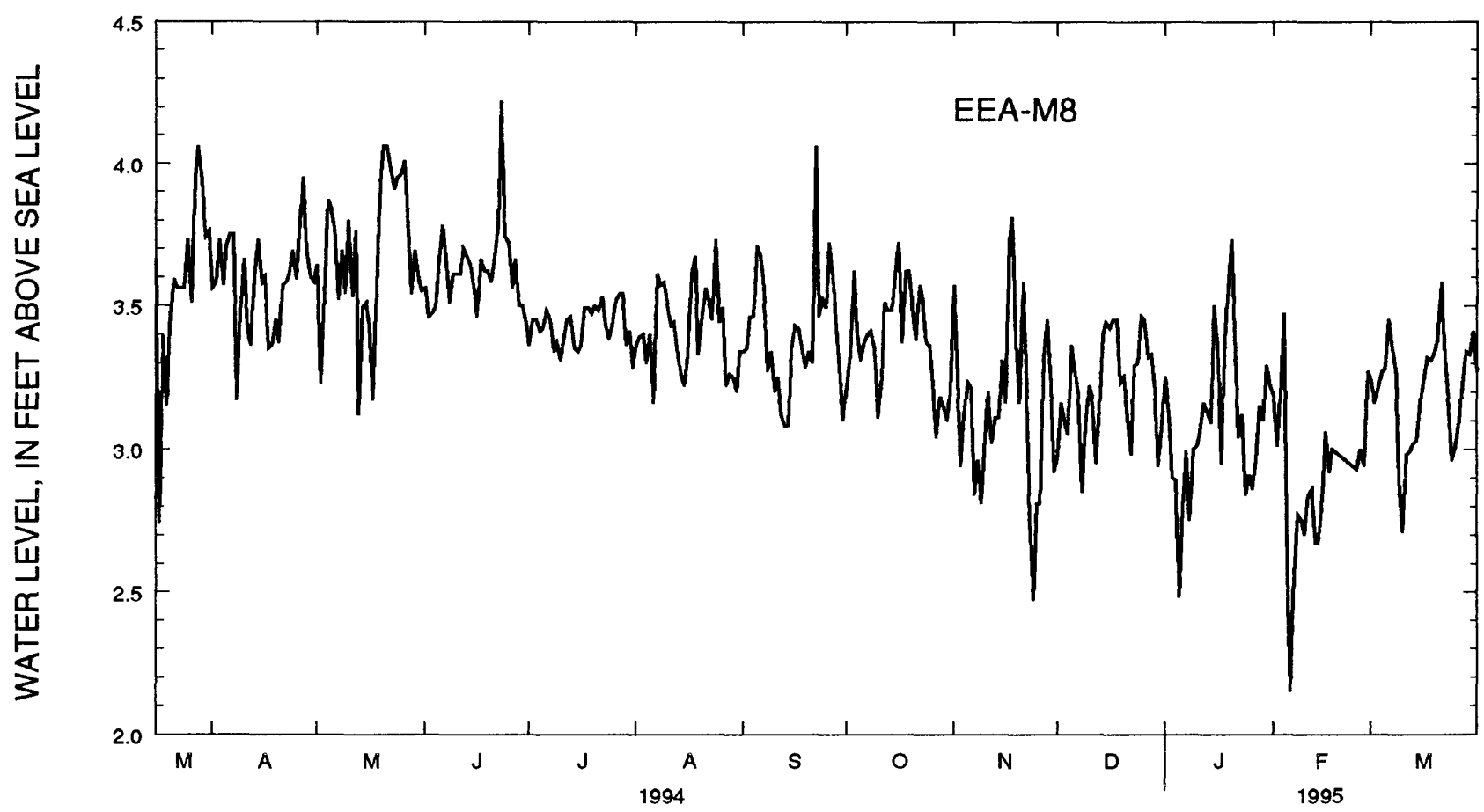

Figure 21. Hydrograph showing minimum daily water level relative to sea level for observation well EEA-M8 at the Explosive Experimental Area, Naval Surface Warfare Center, Dahlgren Site, Dahlgren, Virginia. For location of observation well, see plate 1.

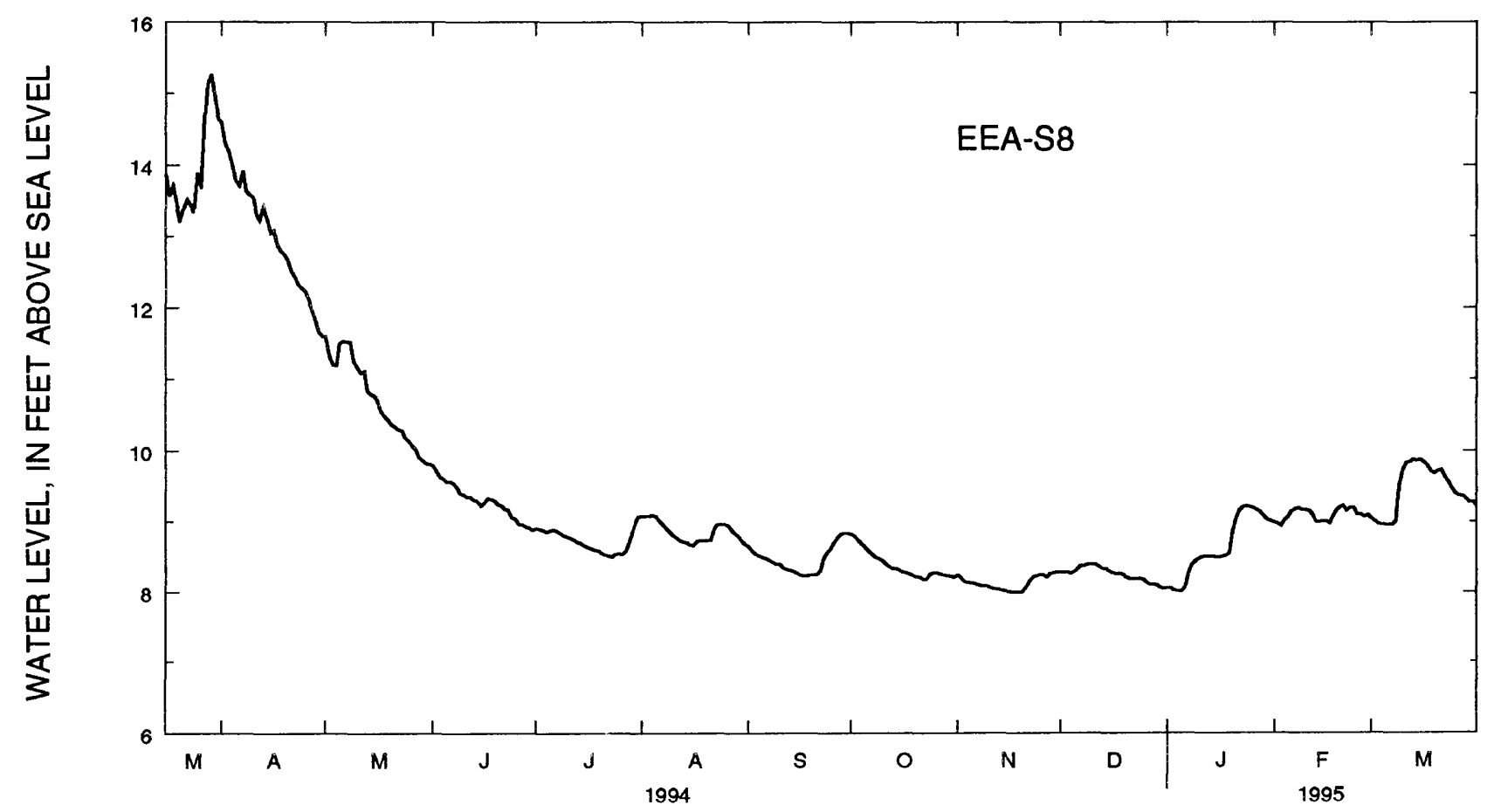

Figure 22. Hydrograph showing minimum daily water level relative to sea level for observation well EEA-S8 at the Explosive Experimental Area, Naval Surface Warfare Center, Dahlgren Site, Dahlgren, Virginia. For location of observation well, see plate 1. 


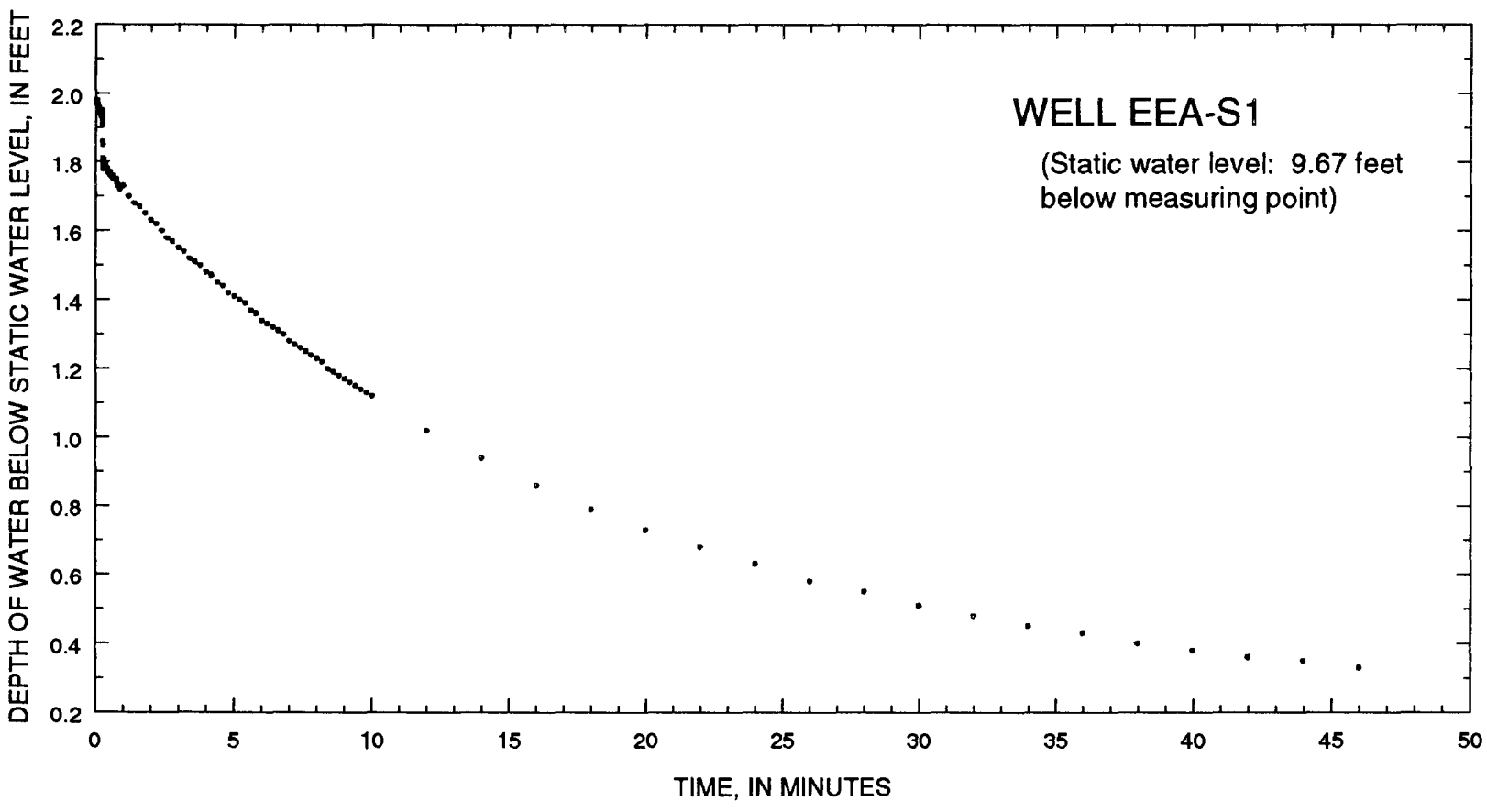

Figure 23. Rising-head data for slug test of observation well EEA-S1 at the Explosive Experimental Area, Naval Surface Warfare Center, Dahlgren Site, Dahlgren, Virginia. For location of observation well, see plate 1.

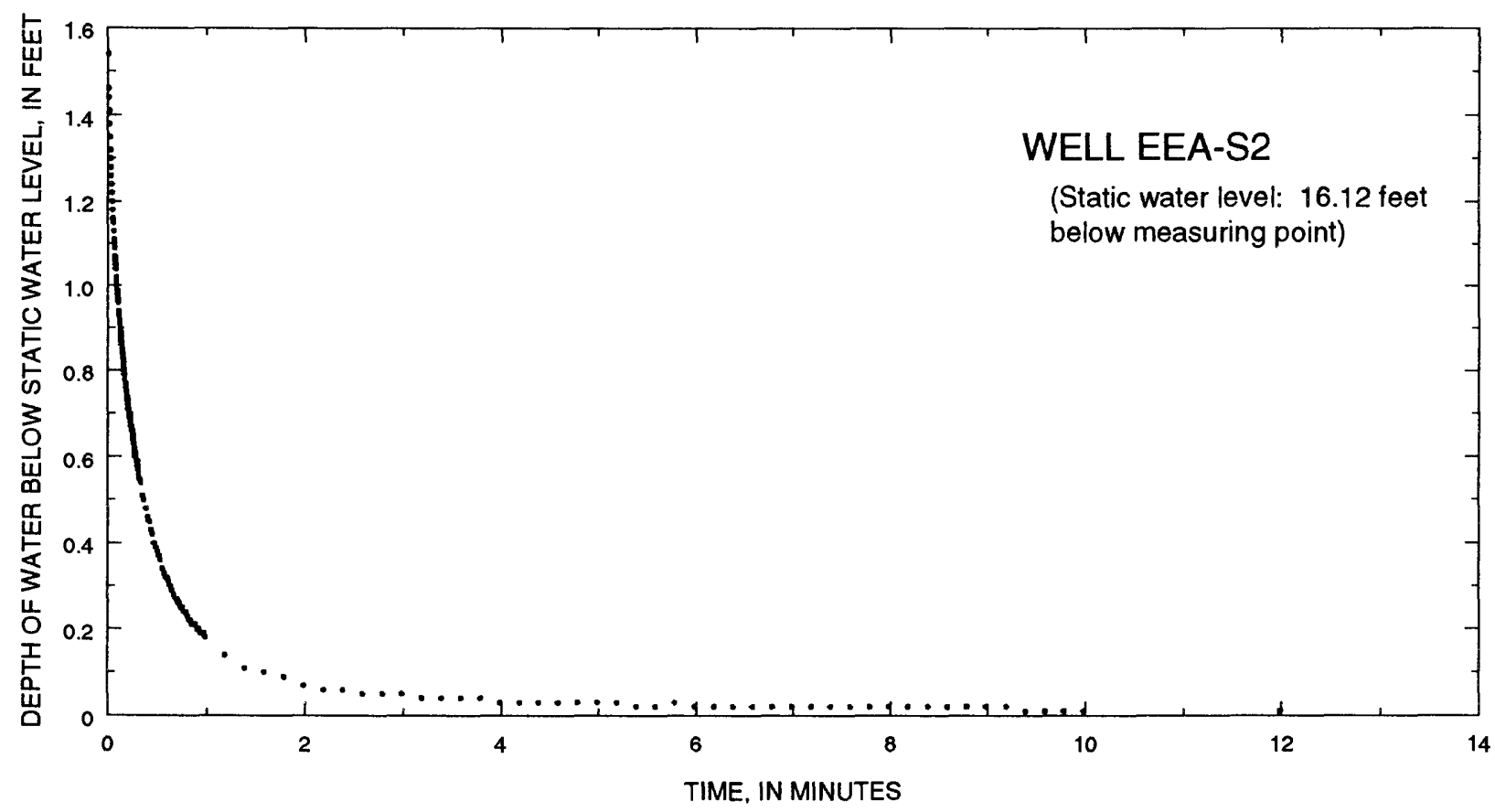

Figure 24. Rising-head data for slug test of observation well EEA-S2 at the Explosive Experimental Area, Naval Surface Warfare Center, Dahlgren Site, Dahlgren, Virginia. For location of observation well, see plate 1. 


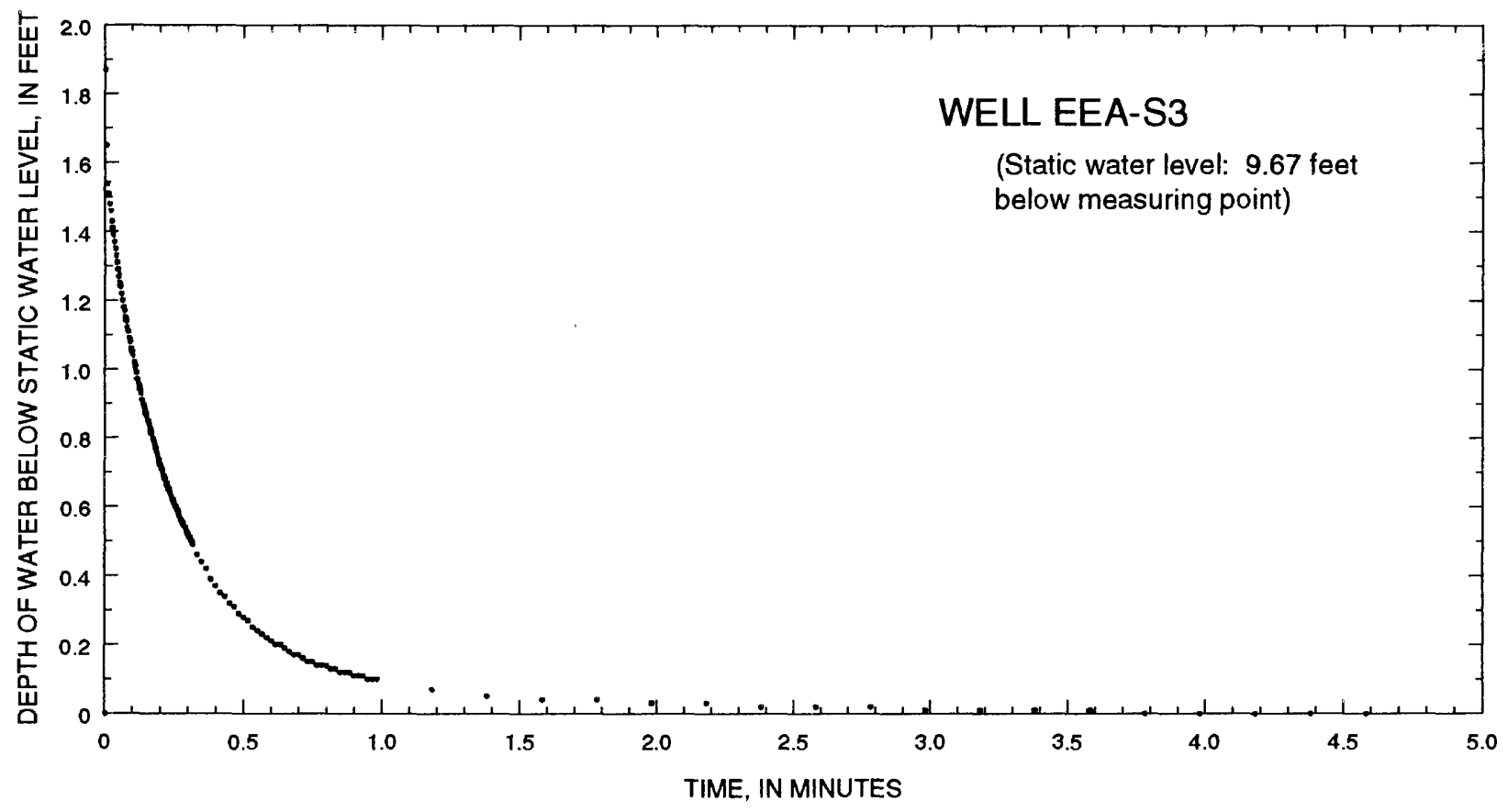

Figure 25. Rising-head data for slug test of observation well EEA-S3 at the Explosive Experimental Area, Naval Surface Warfare Center, Dahlgren Site, Dahlgren, Virginia. For location of observation well, see plate 1.

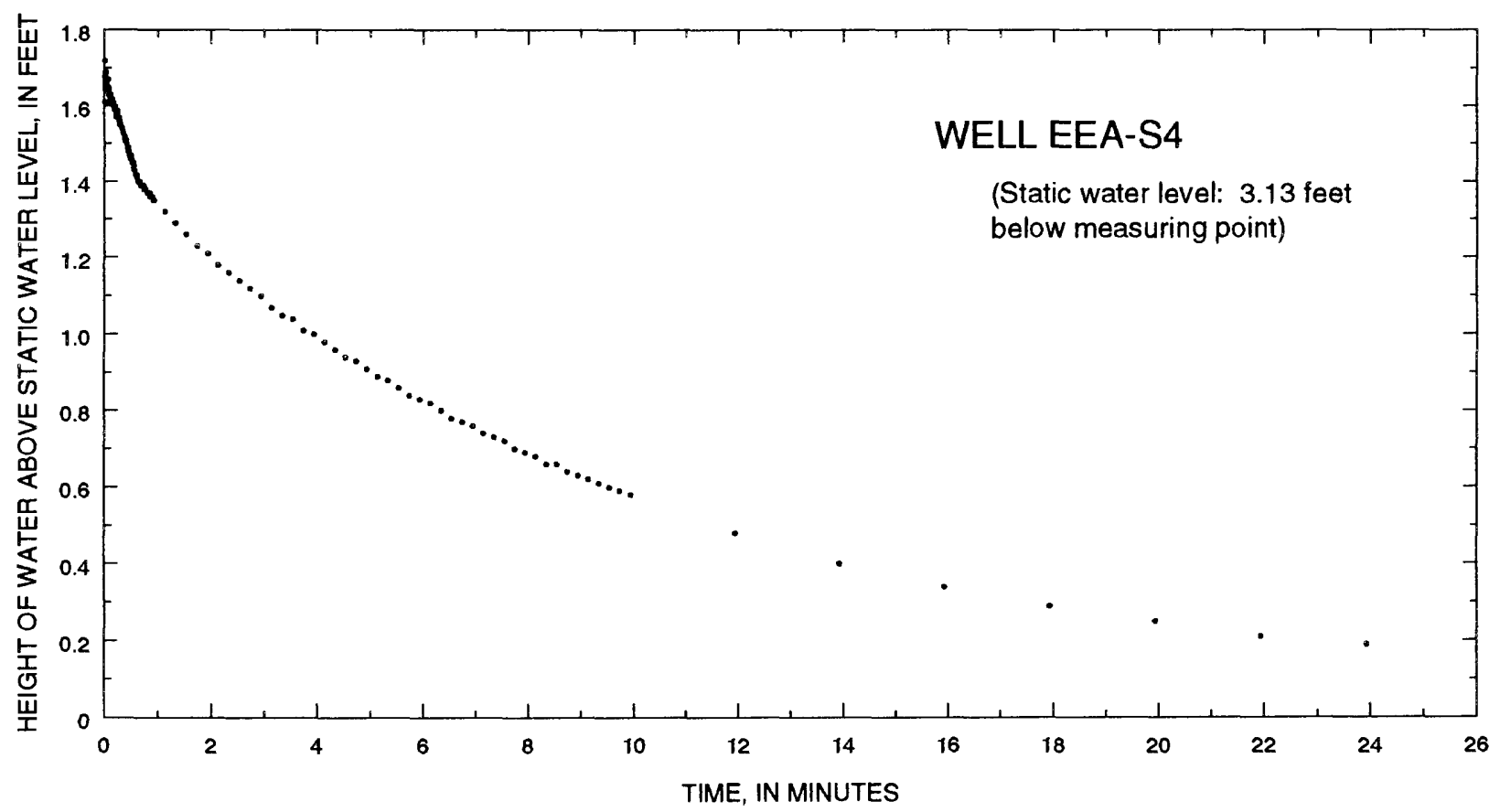

Figure 26. Falling-head data for slug test of observation well EEA-S4 at the Explosive Experimental Area, Naval Surface Warfare Center, Dahlgren Site, Dahlgren, Virginia. For location of observation well, see plate 1. 


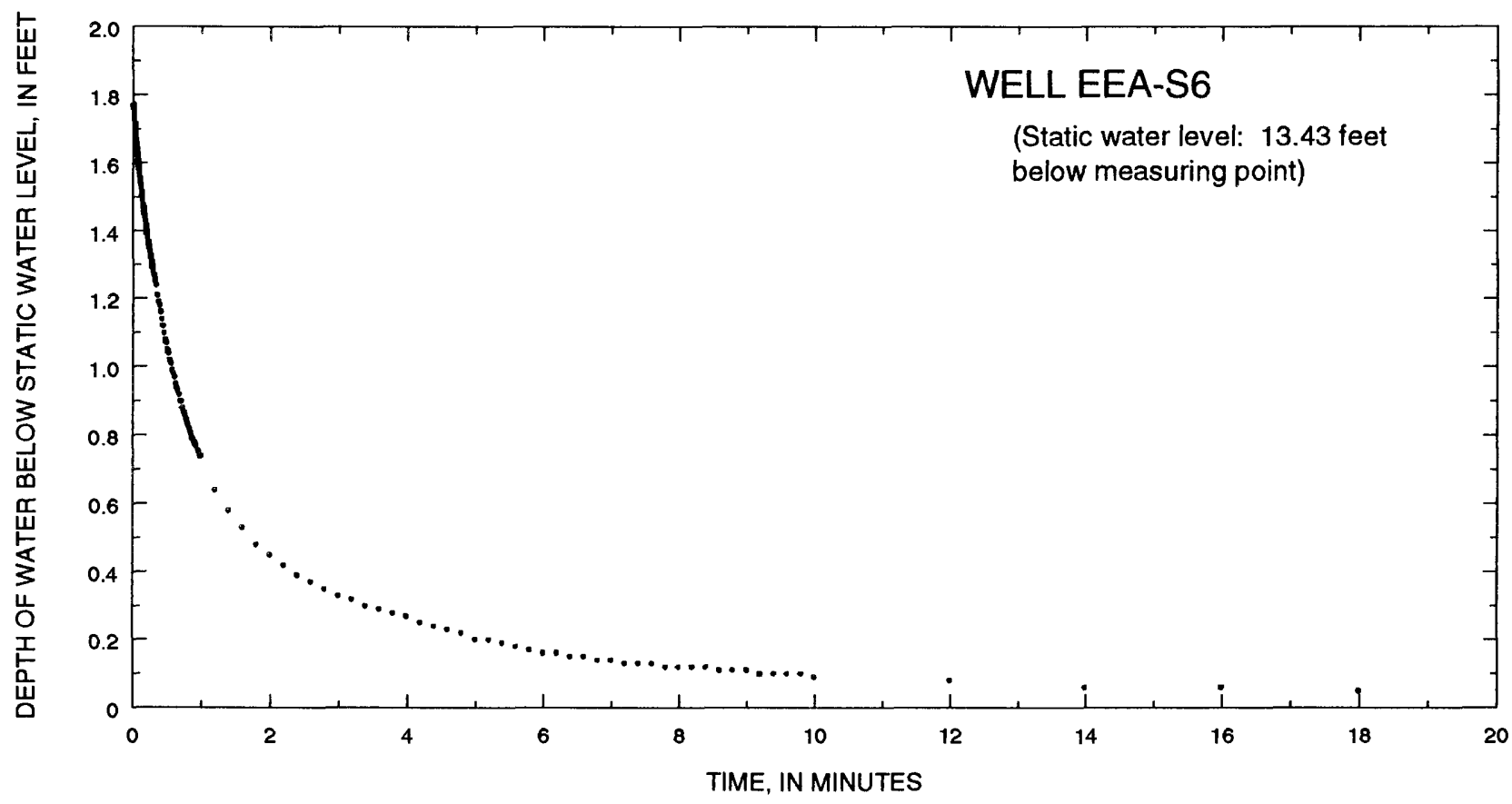

Figure 27. Rising-head data for slug test of observation well EEA-S6 at the Explosive Experimental Area, Naval Surface Warfare Center, Dahlgren Site, Dahlgren, Virginia. For location of observation well, see plate 1.

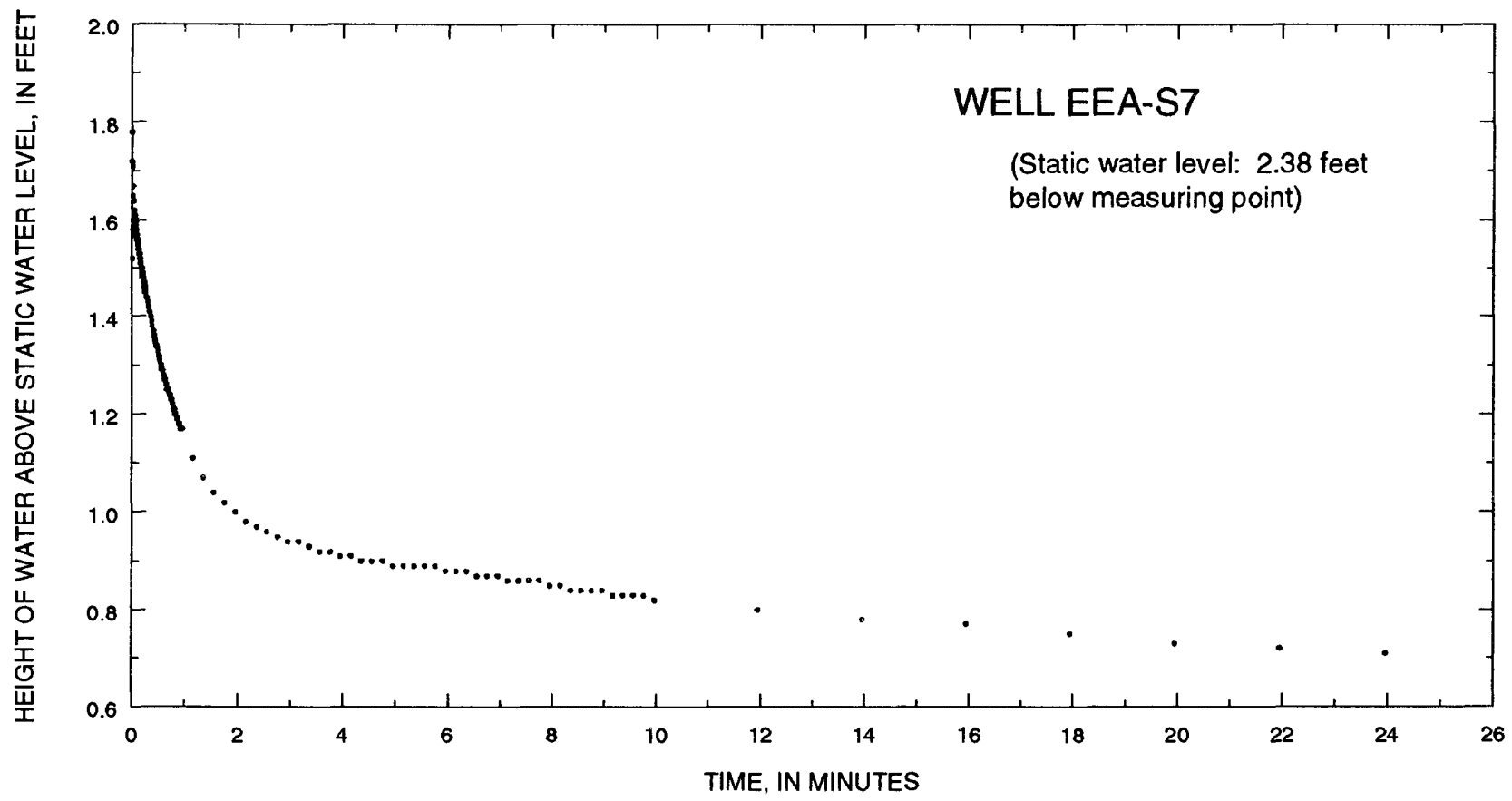

Figure 28. Falling-head data for slug test of observation well EEA-S7 at the Explosive Experimental Area, Naval Surface Warfare Center, Dahlgren Site, Dahlgren, Virginia. For location of observation well, see plate 1. 


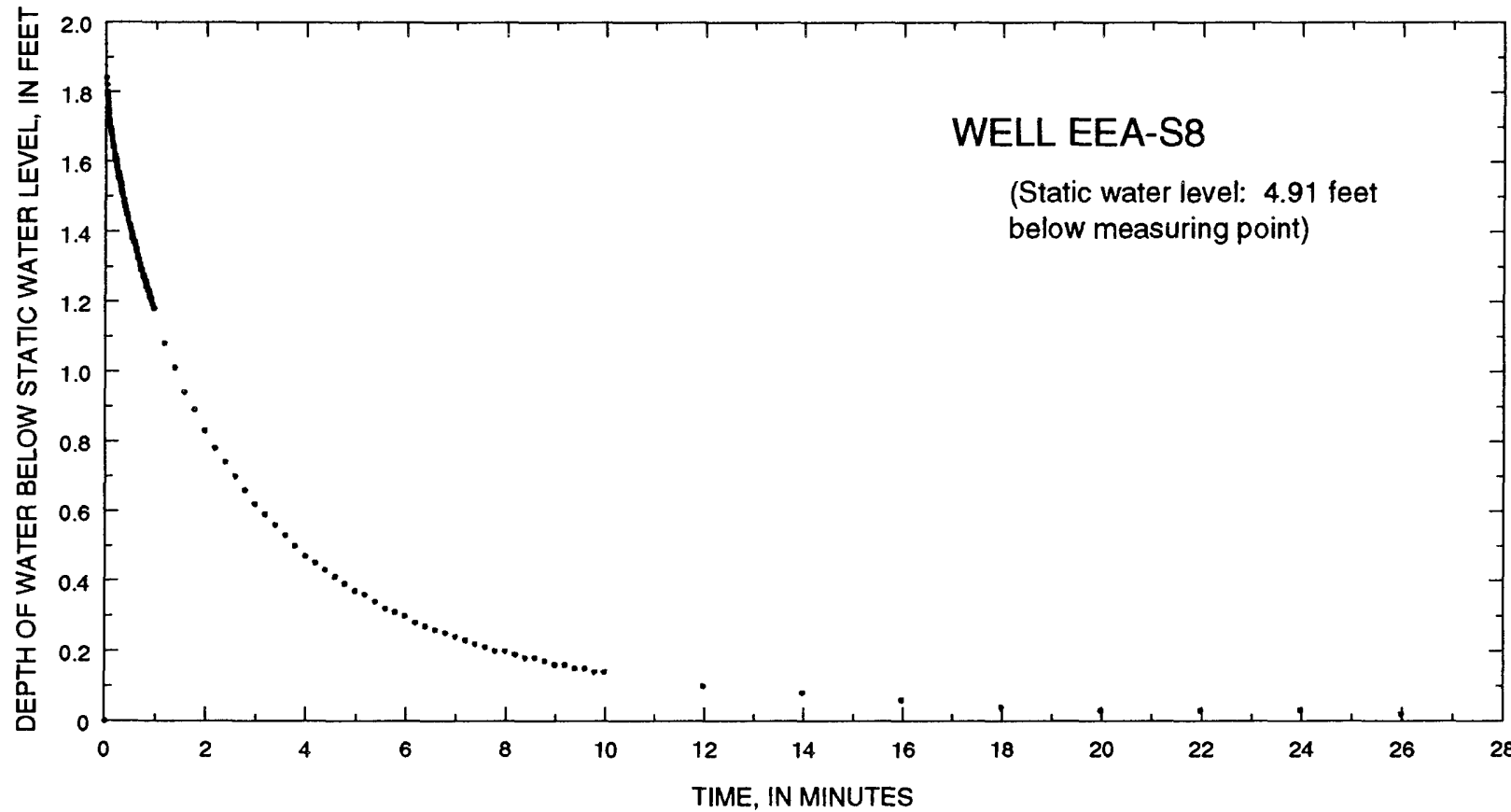

Figure 29. Rising-head data for slug test of observation well EEA-S8 at the Explosive Experimental Area, Naval Surface Warfare Center, Dahlgren Site, Dahlgren, Virginia. For location of observation well, see plate 1.

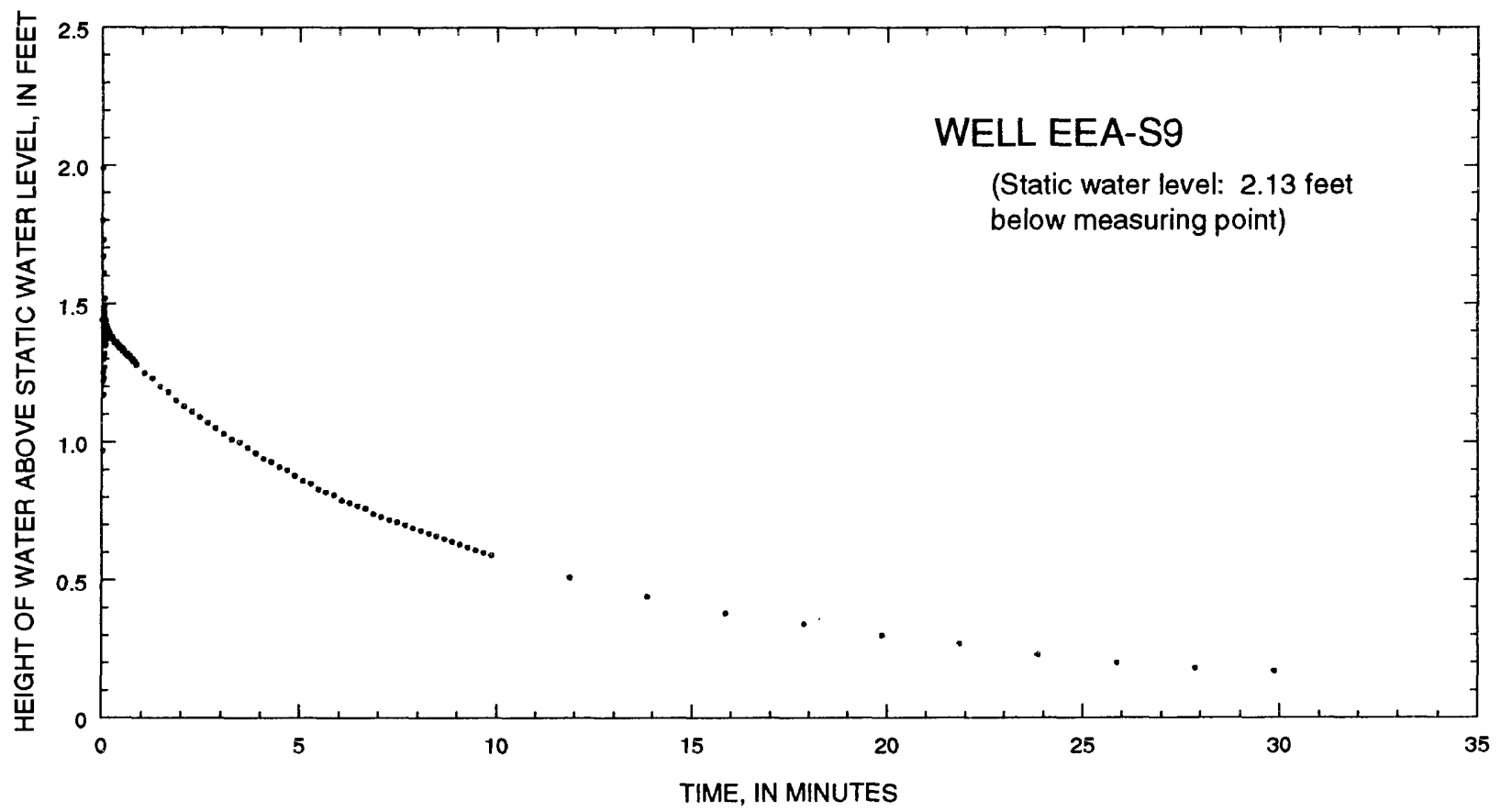

Figure 30. Falling-head data for slug test of observation well EEA-S9 at the Explosive Experimental Area, Naval Surface Warfare Center, Dahlgren Site, Dahlgren, Virginia. For location of observation well, see plate 1. 


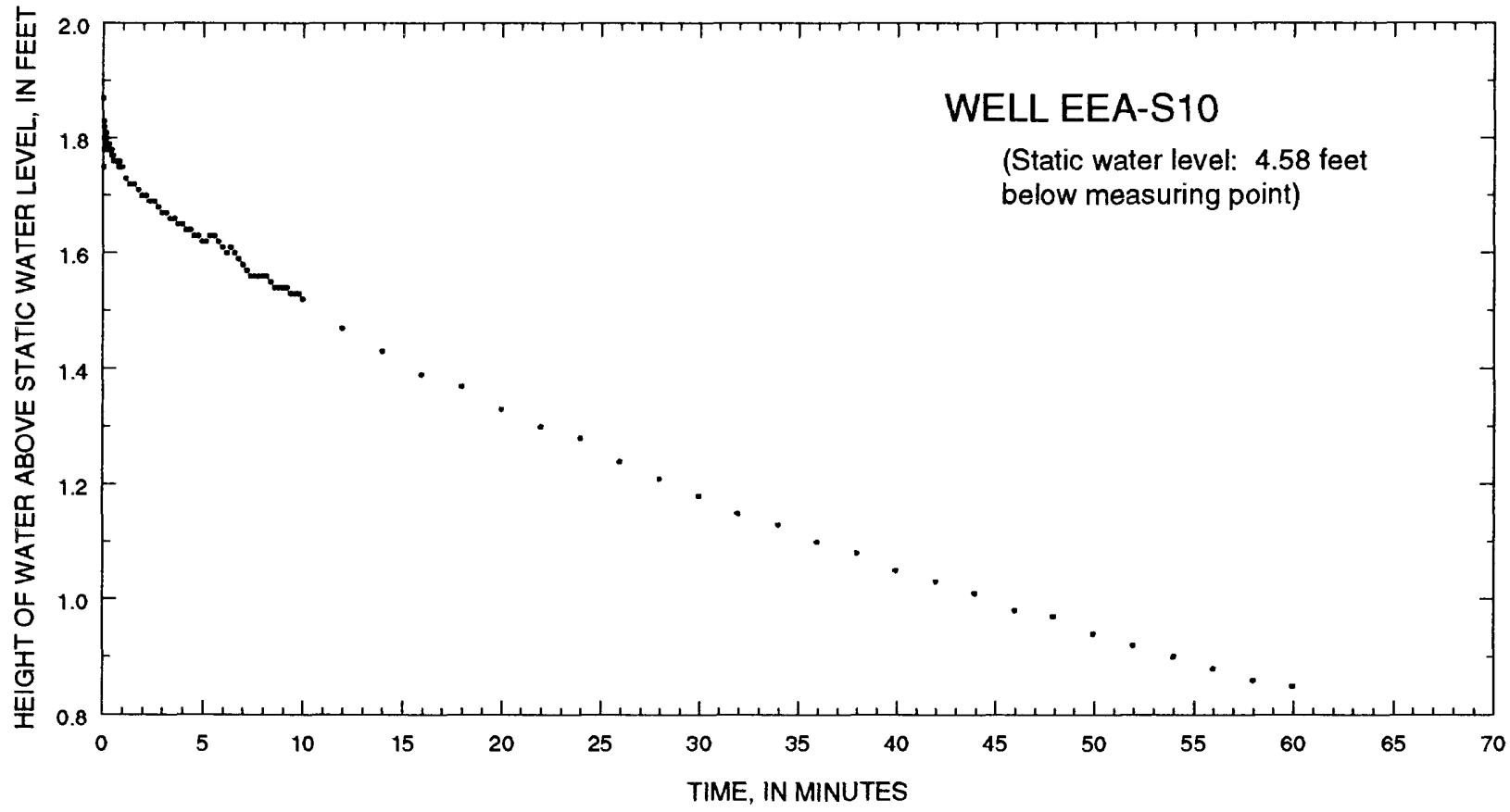

Figure 31. Falling-head data for slug test of observation well EEA-S10 at the Explosive Experimental Area, Naval Warfare Center, Dahlgren Site, Dahlgren, Virginia. For location of observation well, see plate 1.

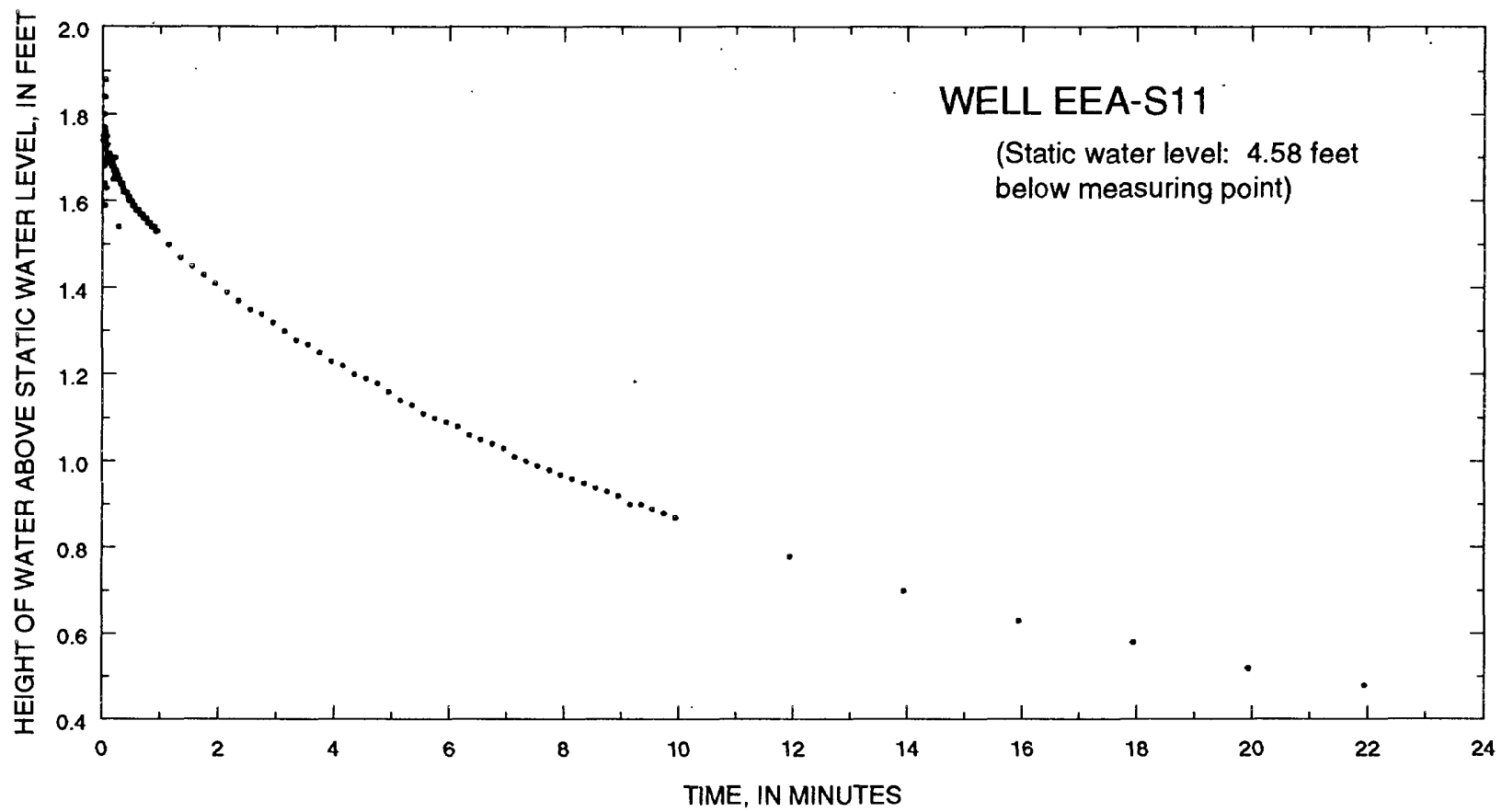

Figure 32. Falling-head data for slug test of observation well EEA-S11 at the Explosive Experimental Area, Naval Warfare Center, Dahlgren Site, Dahlgren, Virginia. For location of observation well, see plate 1. 


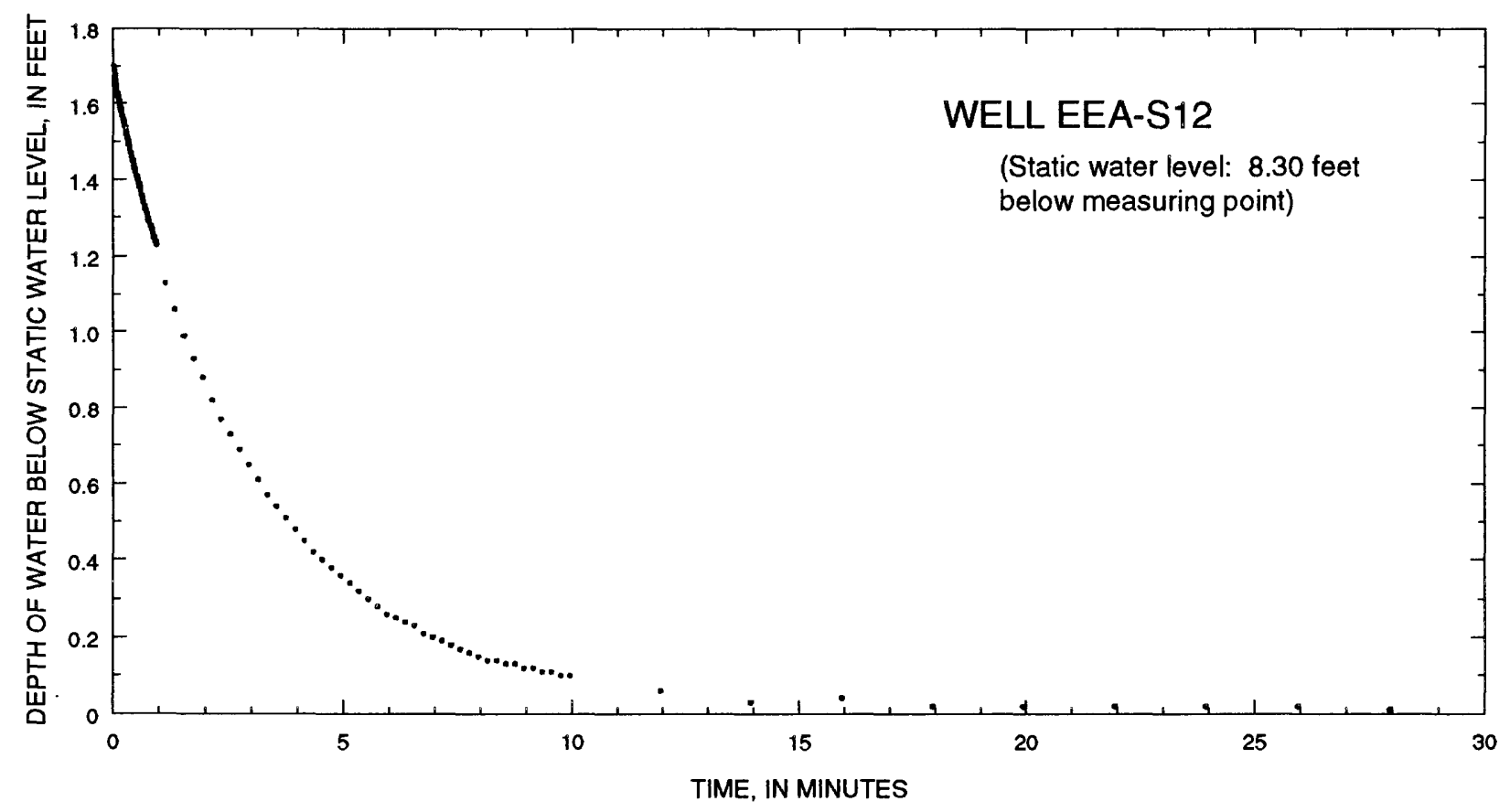

Figure 33. Rising-head data for slug test of observation well EEA-S12 at the Explosive Experimental Area, Naval Surface Warfare Center, Dahlgren Site, Dahlgren, Virginia. For location of observation well, see plate 1.

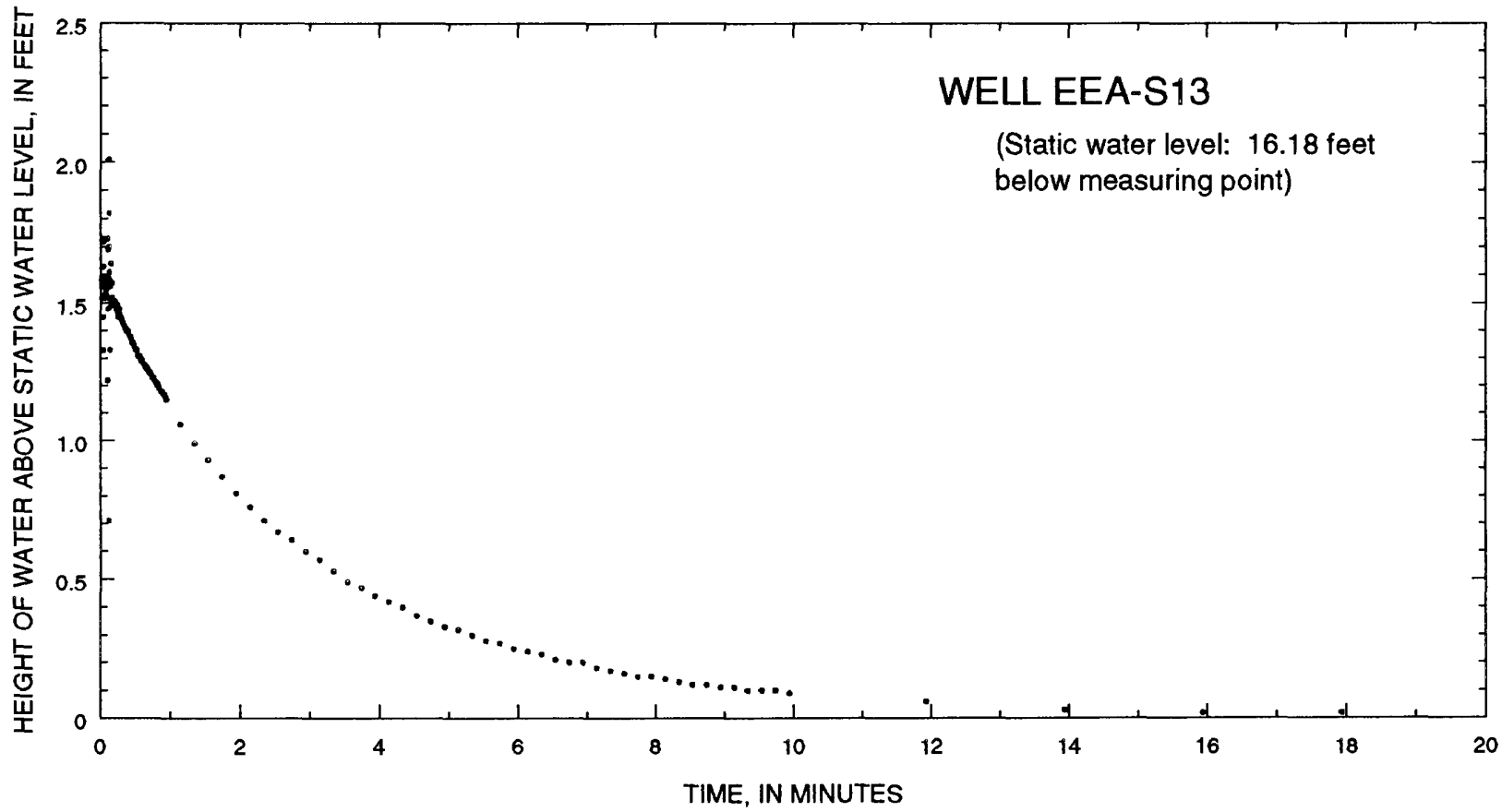

Figure 34. Falling-head data for slug test of observation well EEA-S13 at the Explosive Experimental Area, Naval Surface Warfare Center, Dahlgren Site, Dahlgren, Virginia. For location of observation well, see plate 1. 


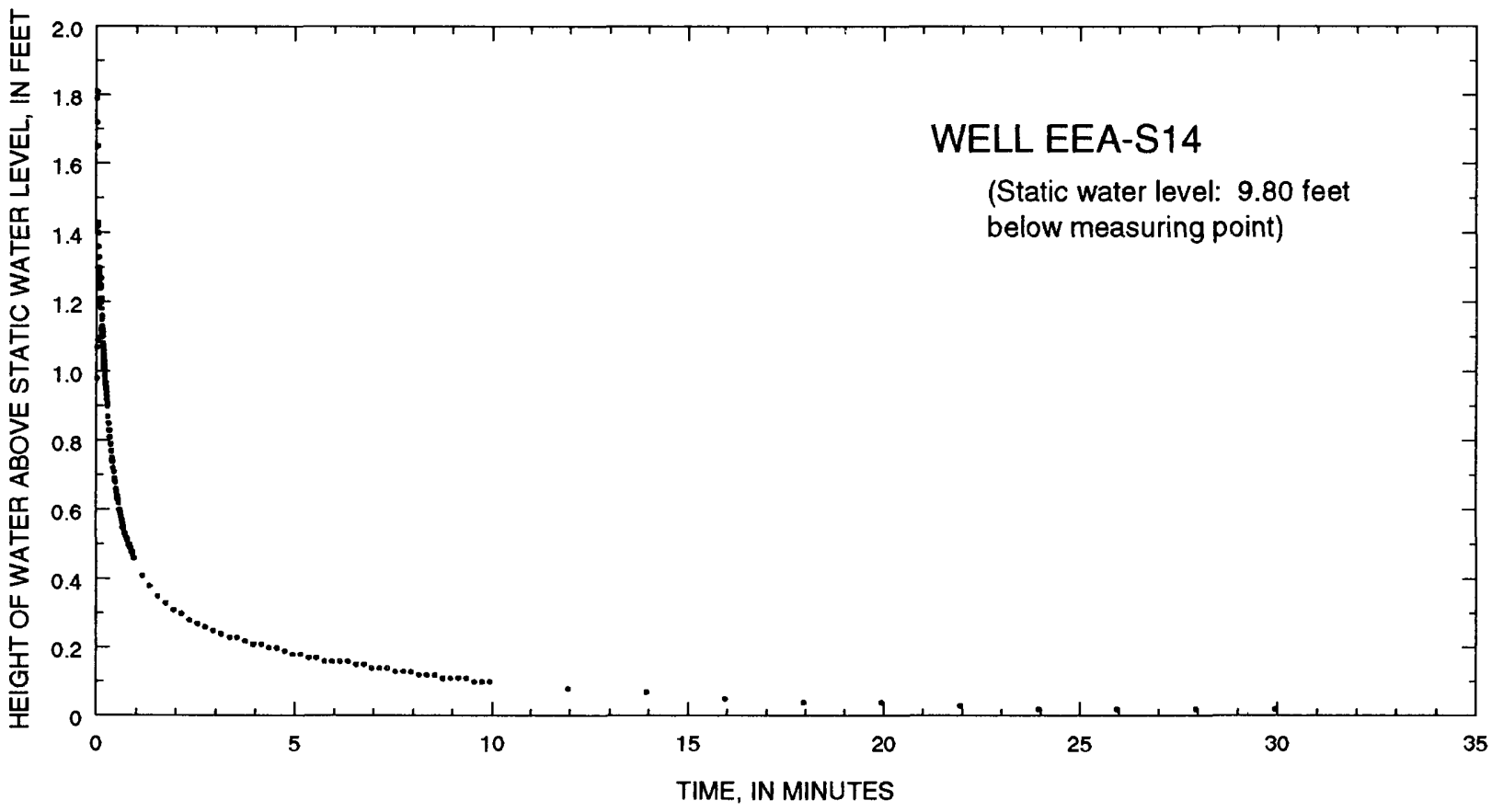

Figure 35. Falling-head data for slug test of observation well EEA-S14 at the Explosive Experimental Area, Naval Surface Warfare Center, Dahlgren Site, Dahlgren, Virginia. For location of observation well, see plate 1.

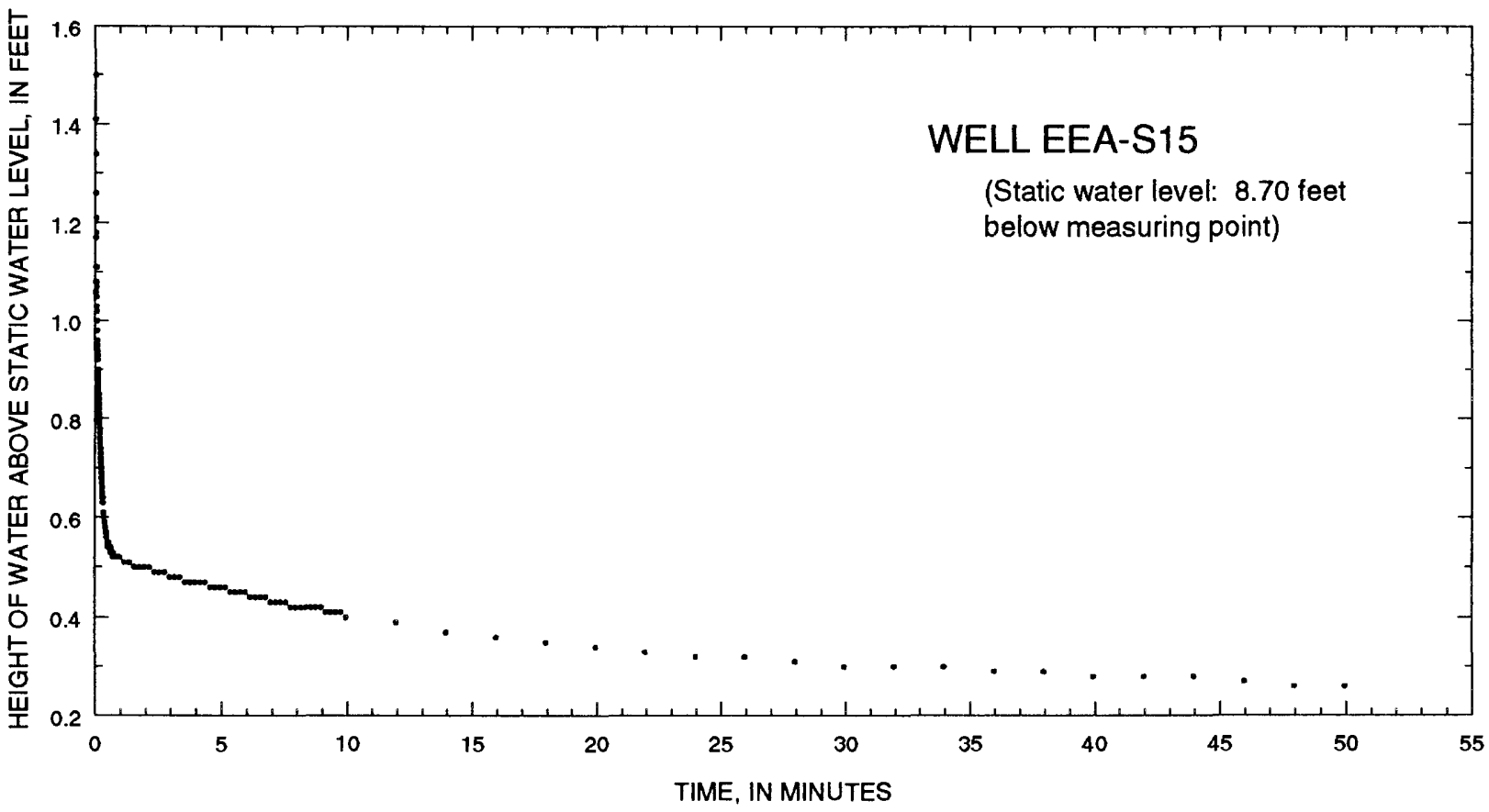

Figure 36. Falling-head data for slug test of observation well EEA-S15 at the Explosive Experimental Area, Naval Surface Warfare Center, Dahlgren Site, Dahlgren, Virginia. For location of observation well, see plate 1. 


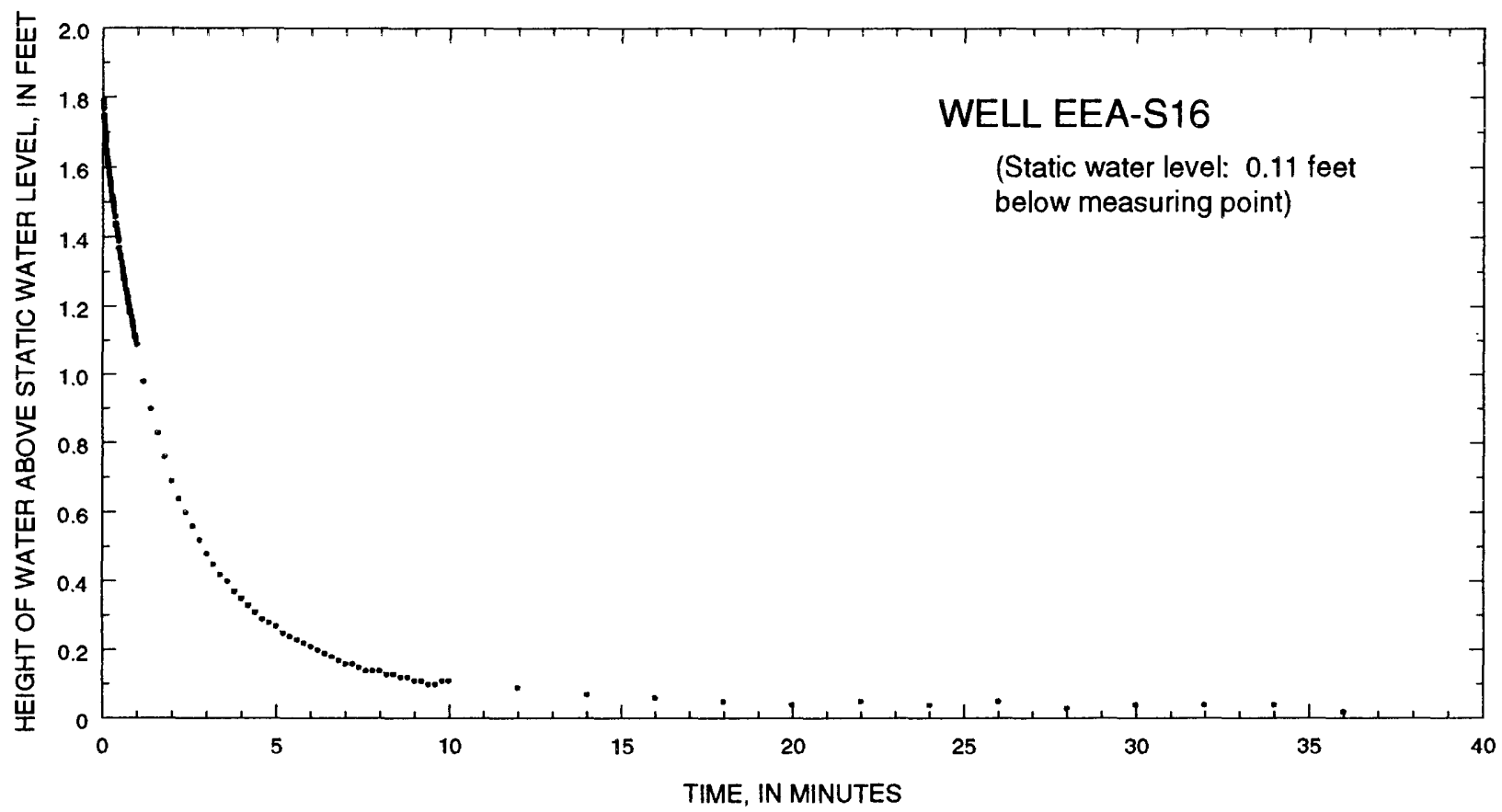

Figure 37. Falling-head data for slug test of observation well EEA-S16 at the Explosive Experimental Area, Naval Surface Warfare Center, Dahlgren Site, Dahlgren, Virginia. For location of observation well, see plate 1.

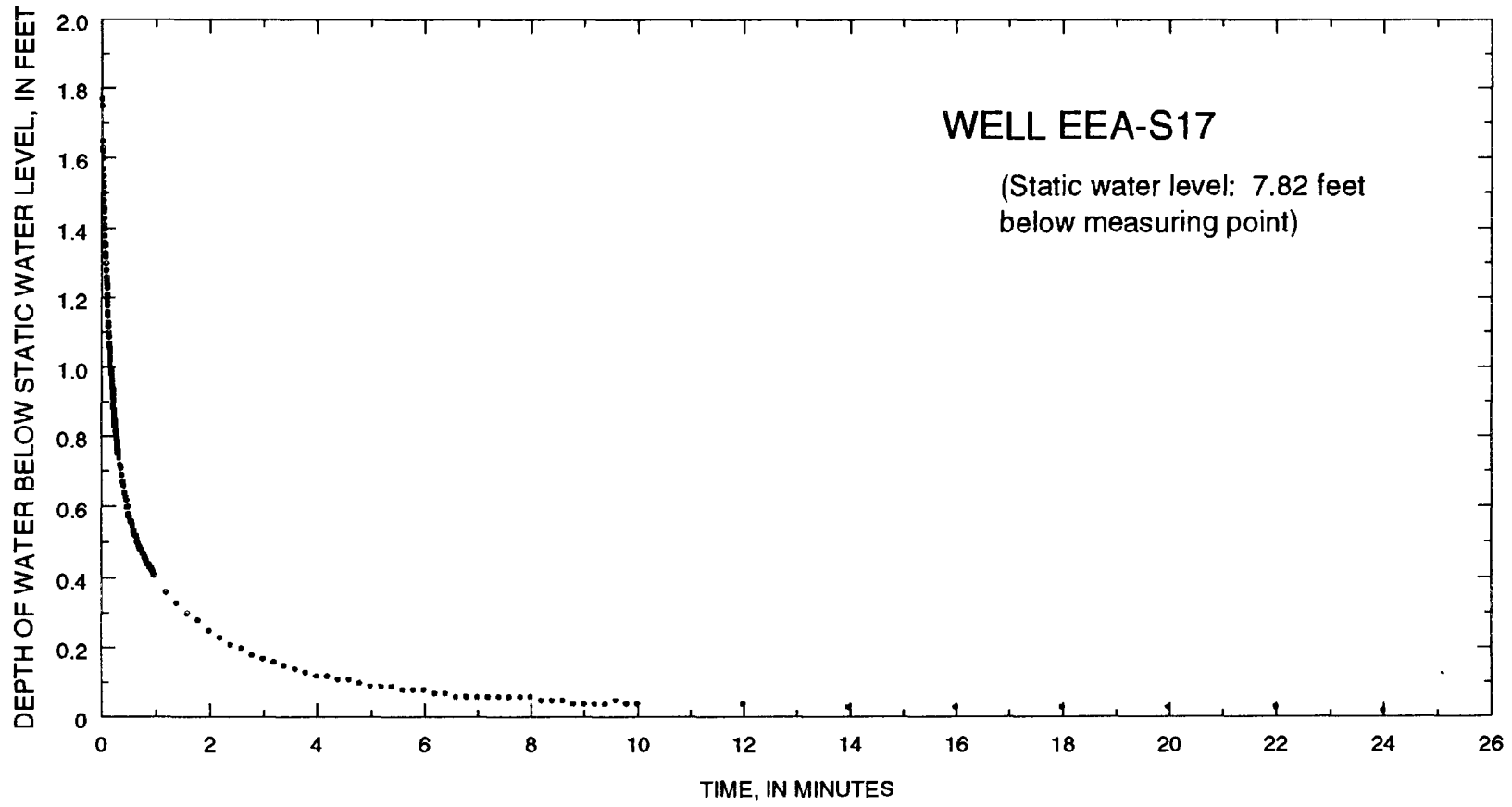

Figure 38. Rising-head data for slug test of observation well EEA-S17 at the Explosive Experimental Area, Naval Surface Warfare Center, Dahlgren Site, Dahlgren, Virginia. For location of observation well, see plate 1. 


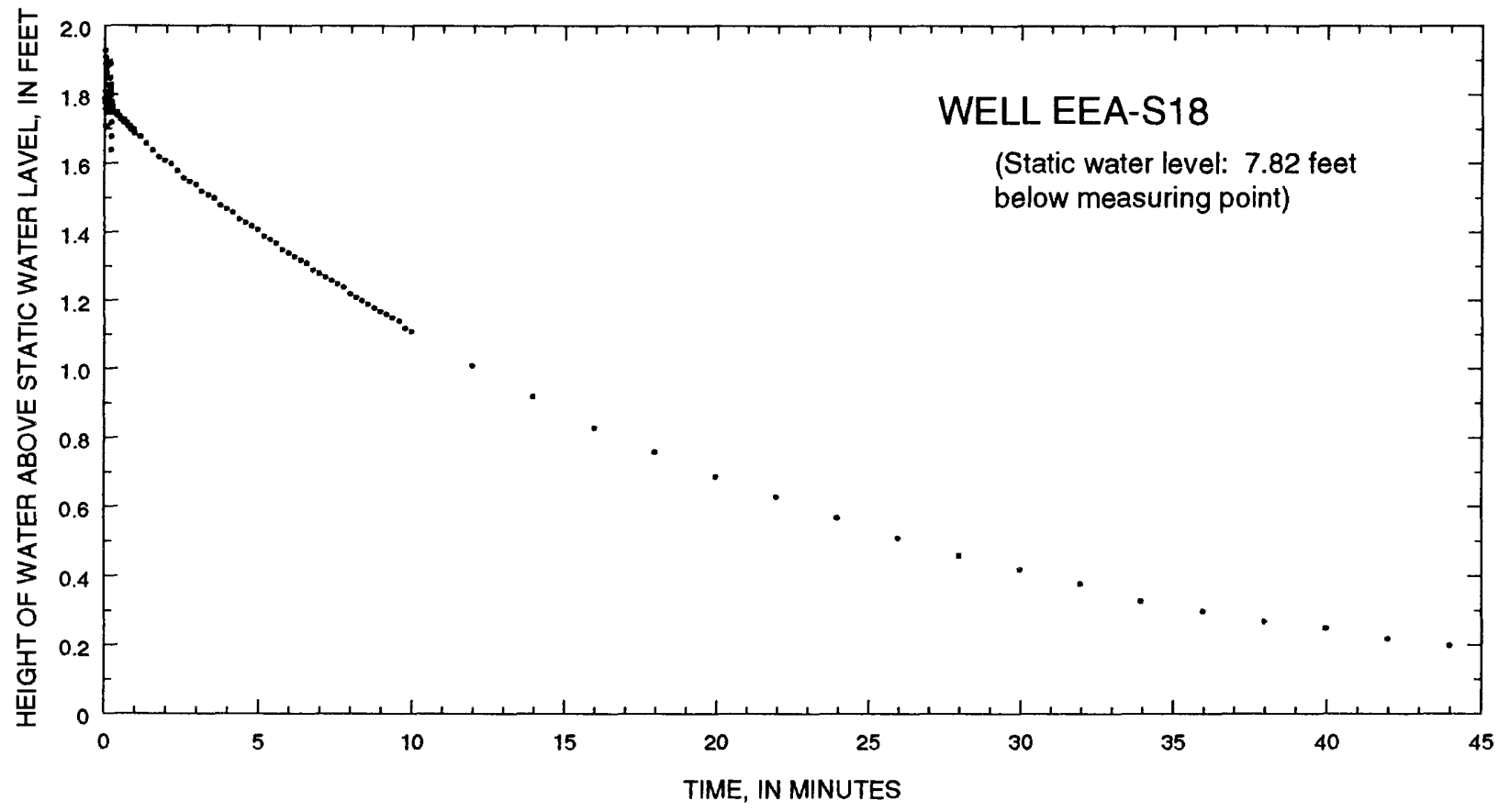

Figure 39. Falling-head data for slug test of observation well EEA-S18 at the Explosive Experimental Area, Naval Surface Warfare Center, Dahlgren Site, Dahlgren, Virginia. For location of observation well, see plate 1.

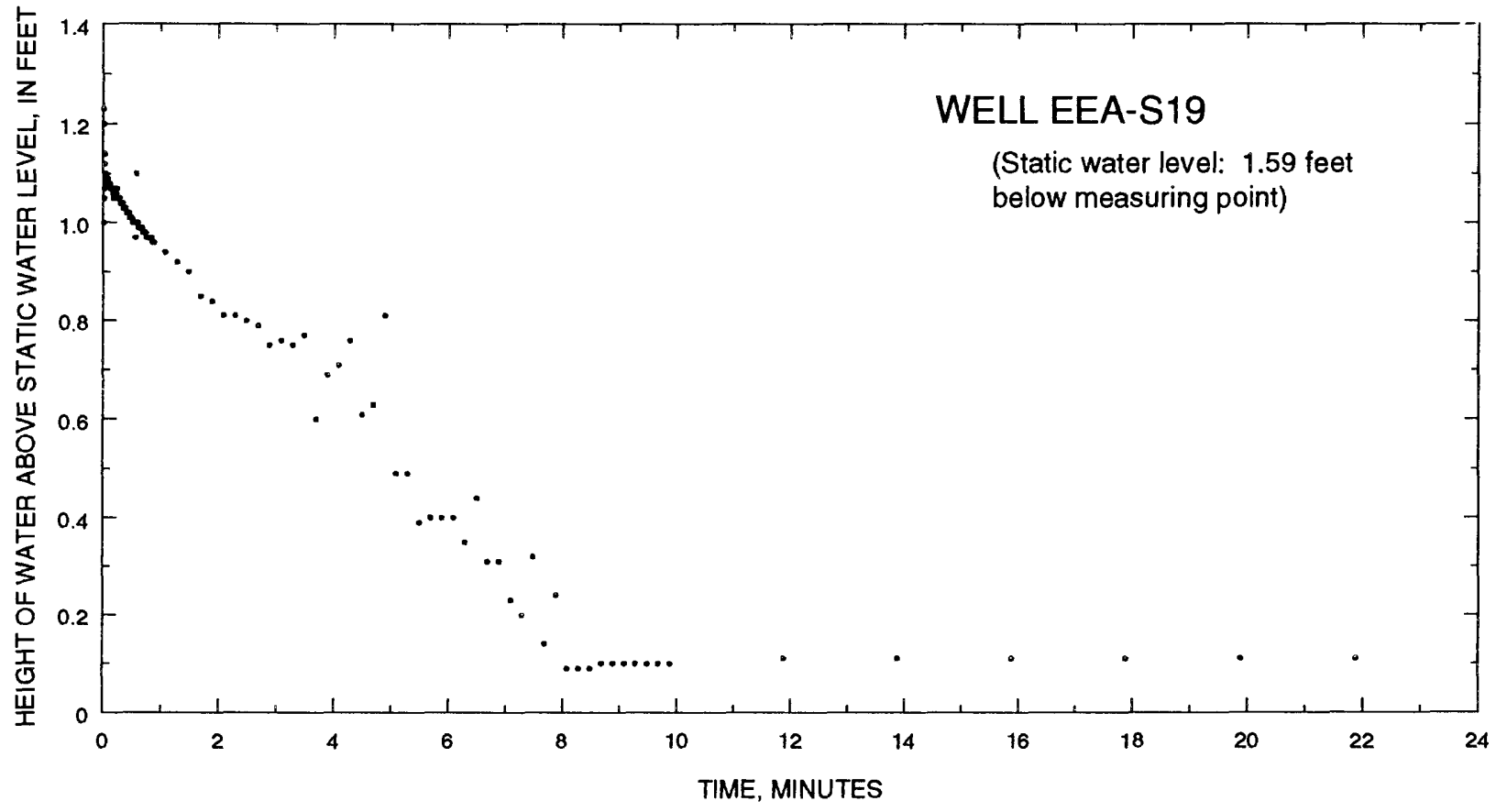

Figure 40. Falling-head data for slug test of observation well EEA-S19 at the Explosive Experimental Area, Naval Surface Warfare Center, Dahlgren Site, Dahlgren, Virginia. For location of observation well, see plate 1. 


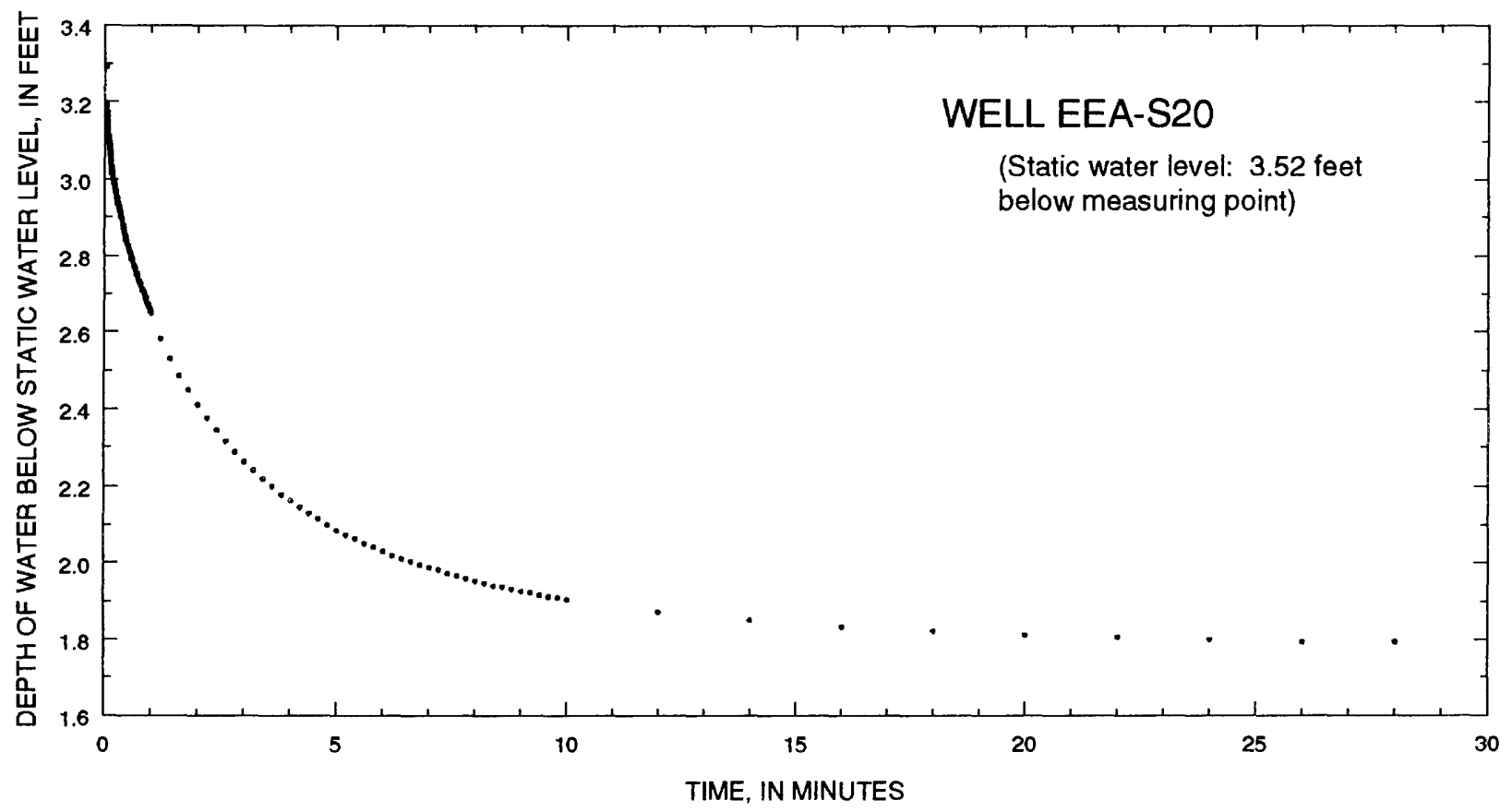

Figure 41. Rising-head data for slug test of observation well EEA-S20 at the Explosive Experimental Area, Naval Surface Warfare Center, Dahlgren Site, Dahlgren, Virginia. For location of observation well, see plate 1.

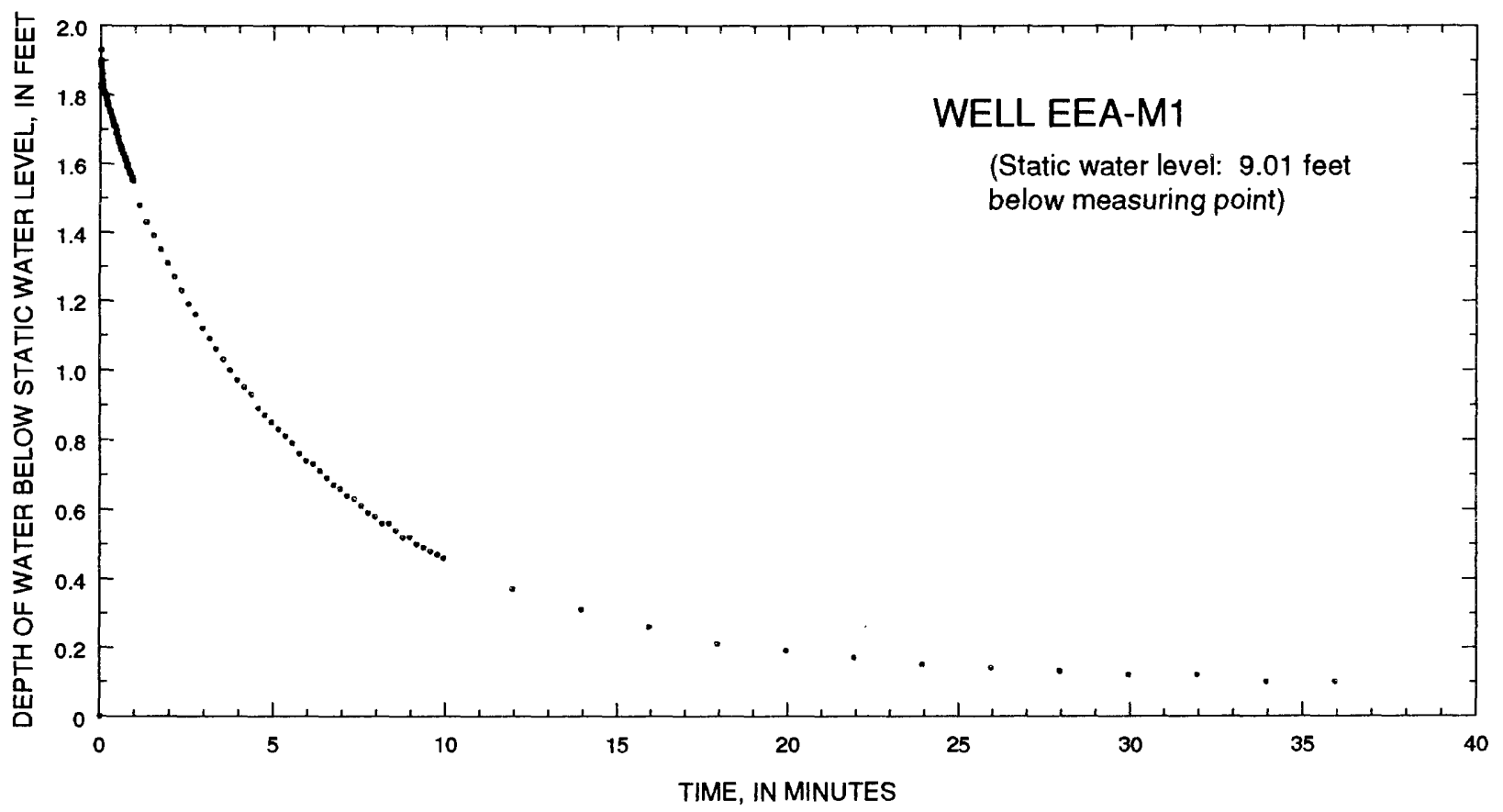

Figure 42. Rising-head data for slug test of observation well EEA-M1 at the Explosive Experimental Area, Naval Surface Warfare Center, Dahigren Site, Dahlgren, Virginia. For location of observation well, see plate 1. 


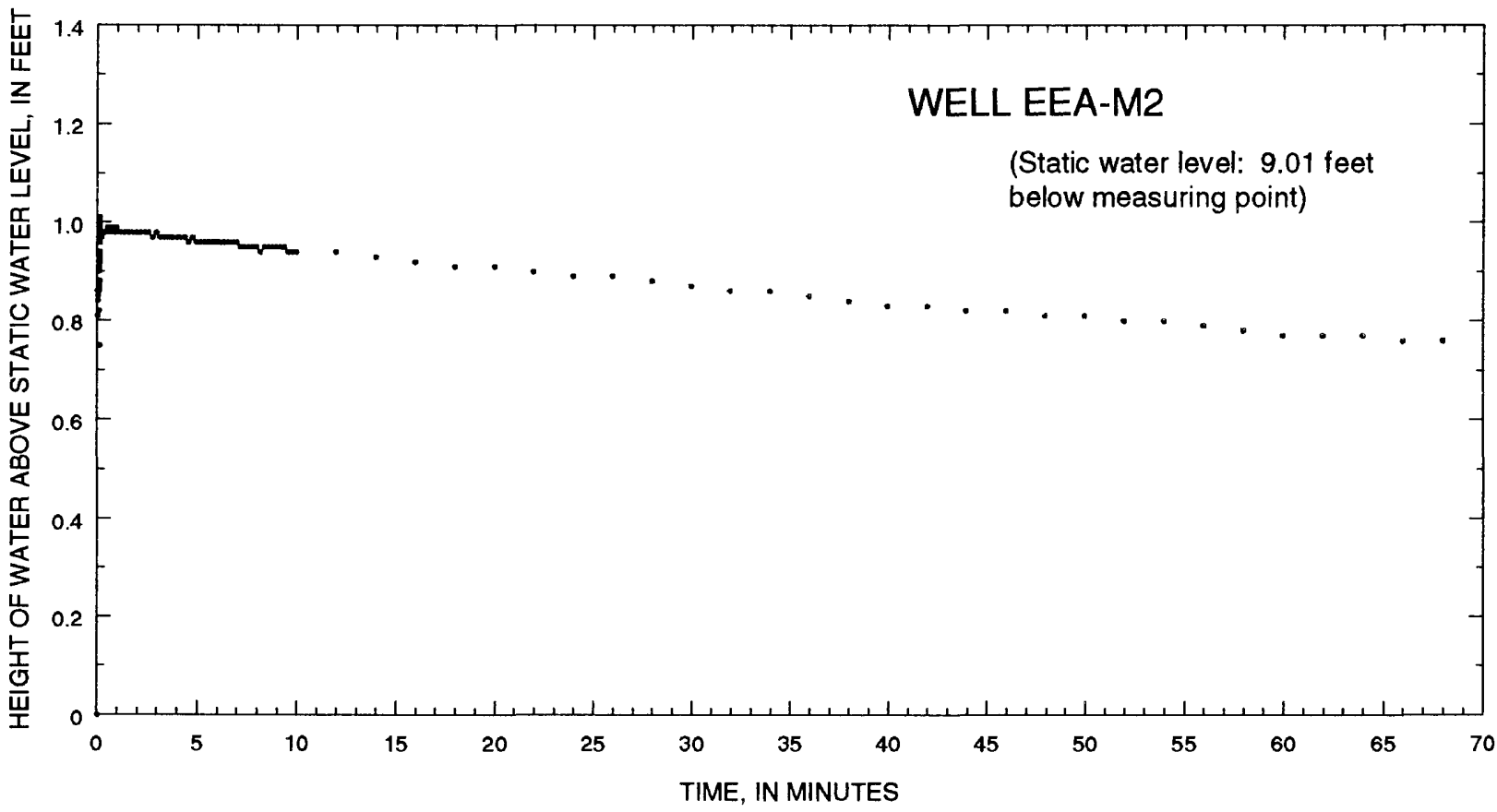

Figure 43. Falling-head data for slug test of observation well EEA-M2 at the Explosive Experimental Area, Naval Surface Warfare Center, Dahlgren Site, Dahlgren, Virginia. For location of observation well, see plate 1.

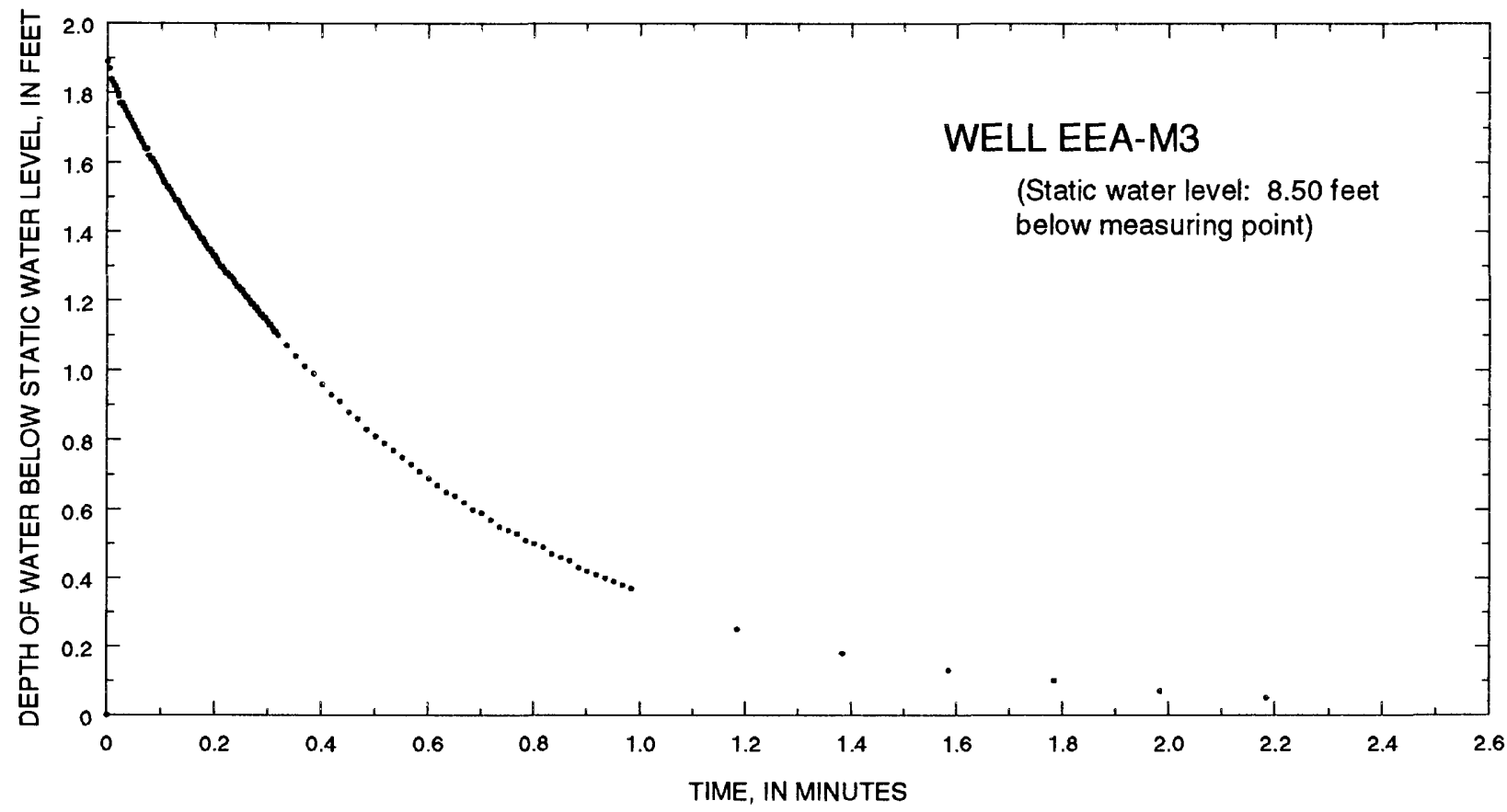

Figure 44. Rising-head data for slug test of observation well EEA-M3 at the Explosive Experimental Area, Naval Surface Warfare Center, Dahlgren Site, Dahlgren, Virginia. For location of observation well, see plate 1. 


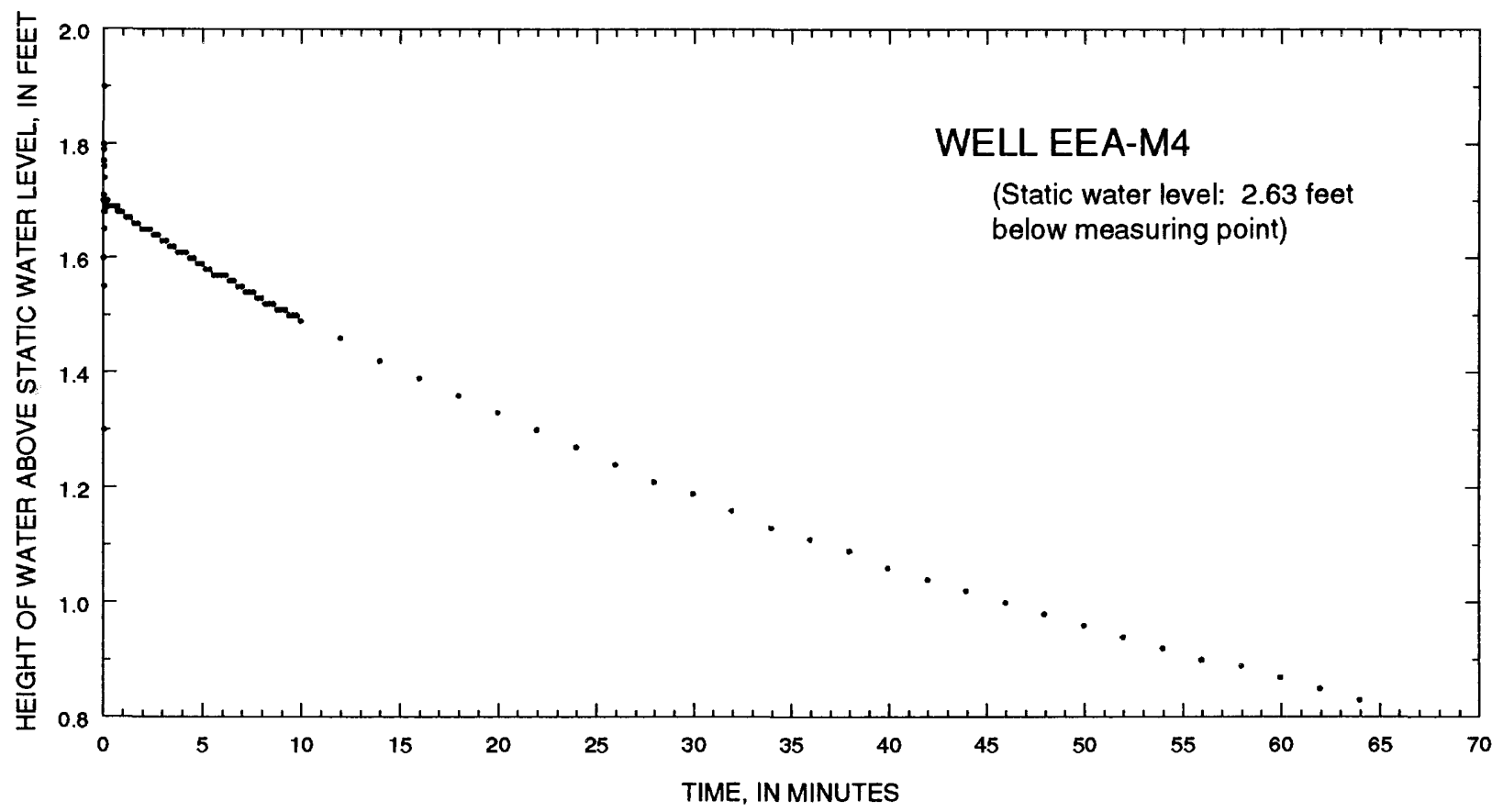

Figure 45. Falling-head data for slug test of observation well EEA-M4 at the Explosive Experimental Area, Naval Surface Warfare Center, Dahlgren Site, Dahlgren, Virginia. For location of observation well, see plate 1.

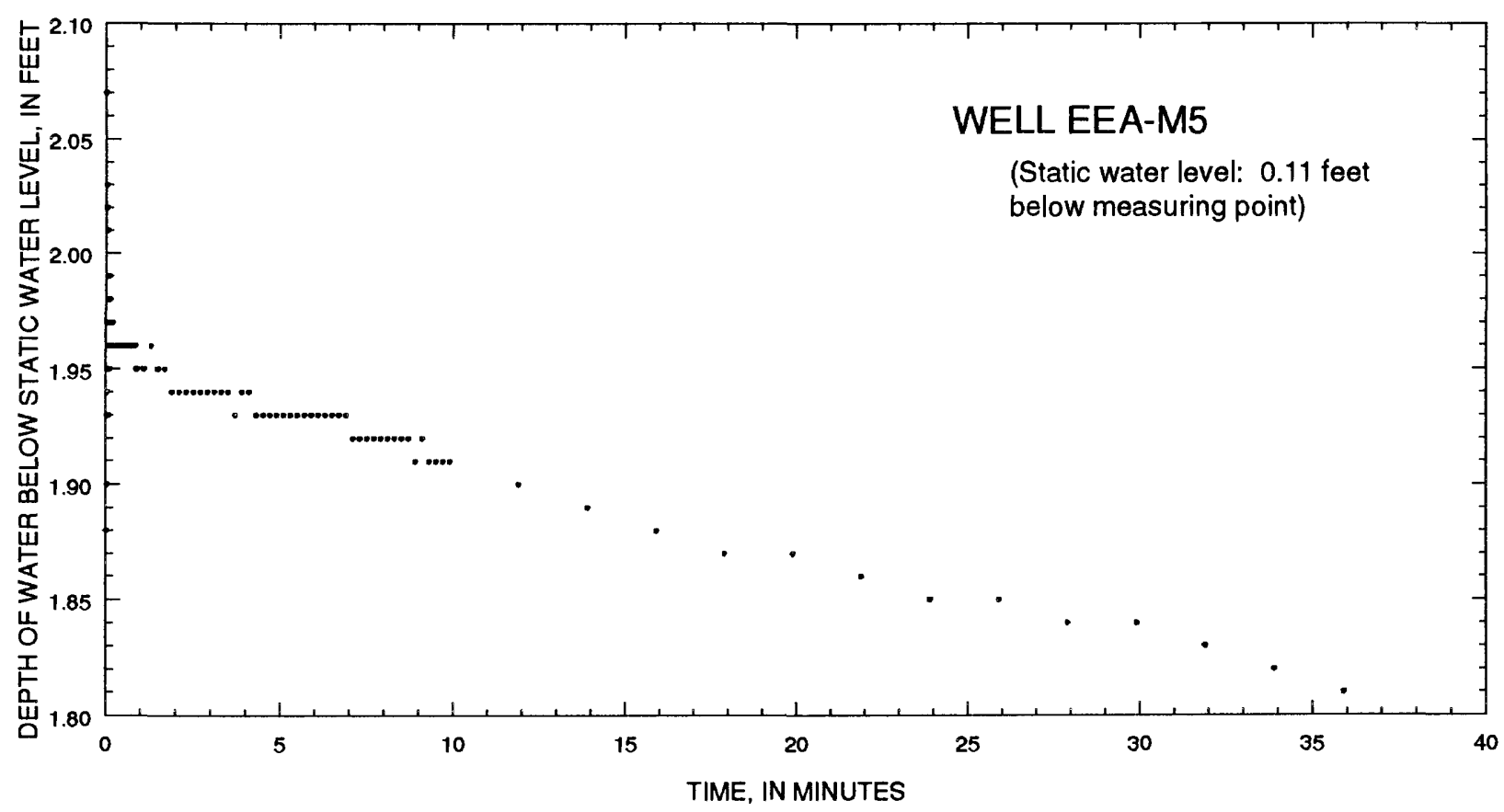

Figure 46. Rising-head data for slug test of observation well EEA-M5 at the Explosive Experimental Area, Naval Surface Warfare Center, Dahlgren Site, Dahlgren, Virginia. For location of observation well, see plate 1. 


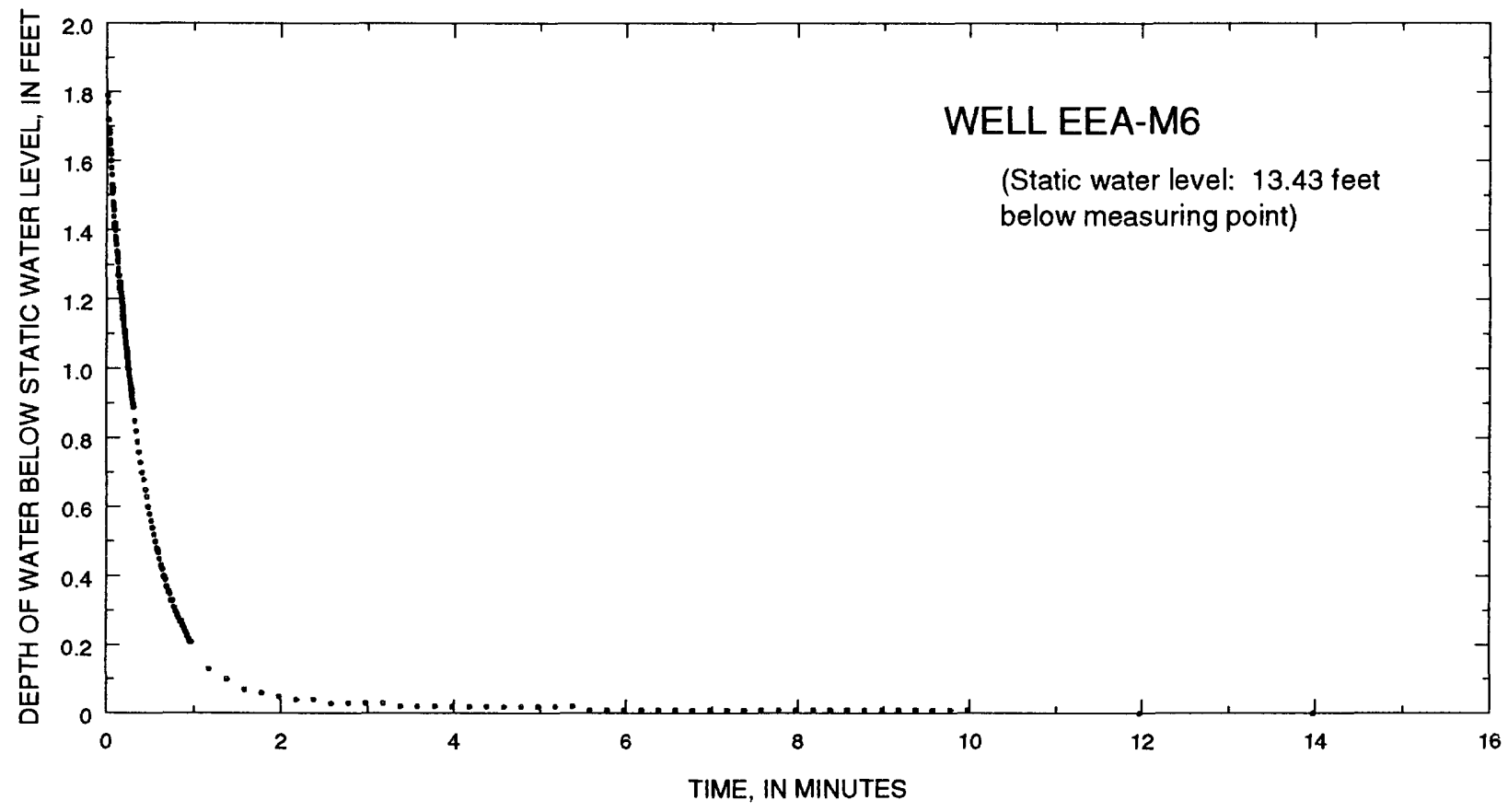

Figure 47. Rising-head data for slug test of observation well EEA-M6 at the Explosive Experimental Area, Naval Surface Warfare Center, Dahlgren Site, Dahlgren, Virginia. For location of observation well, see plate 1.

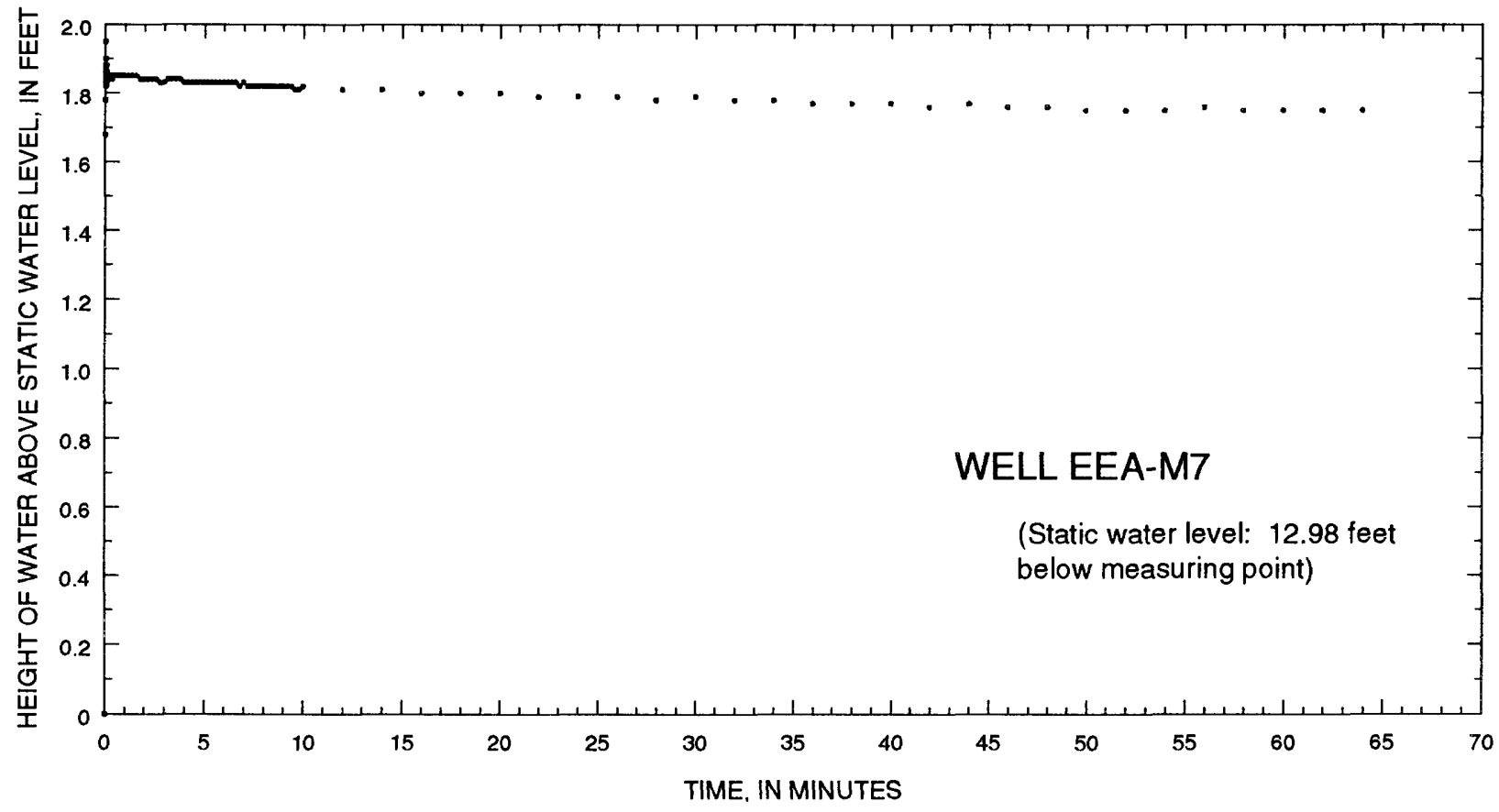

Figure 48. Falling-head data for slug test of observation well EEA-M7 at the Explosive Experimental Area, Naval Surface Warfare Center, Dahlgren Site, Dahlgren, Virginia. For location of observation well, see plate 1. 


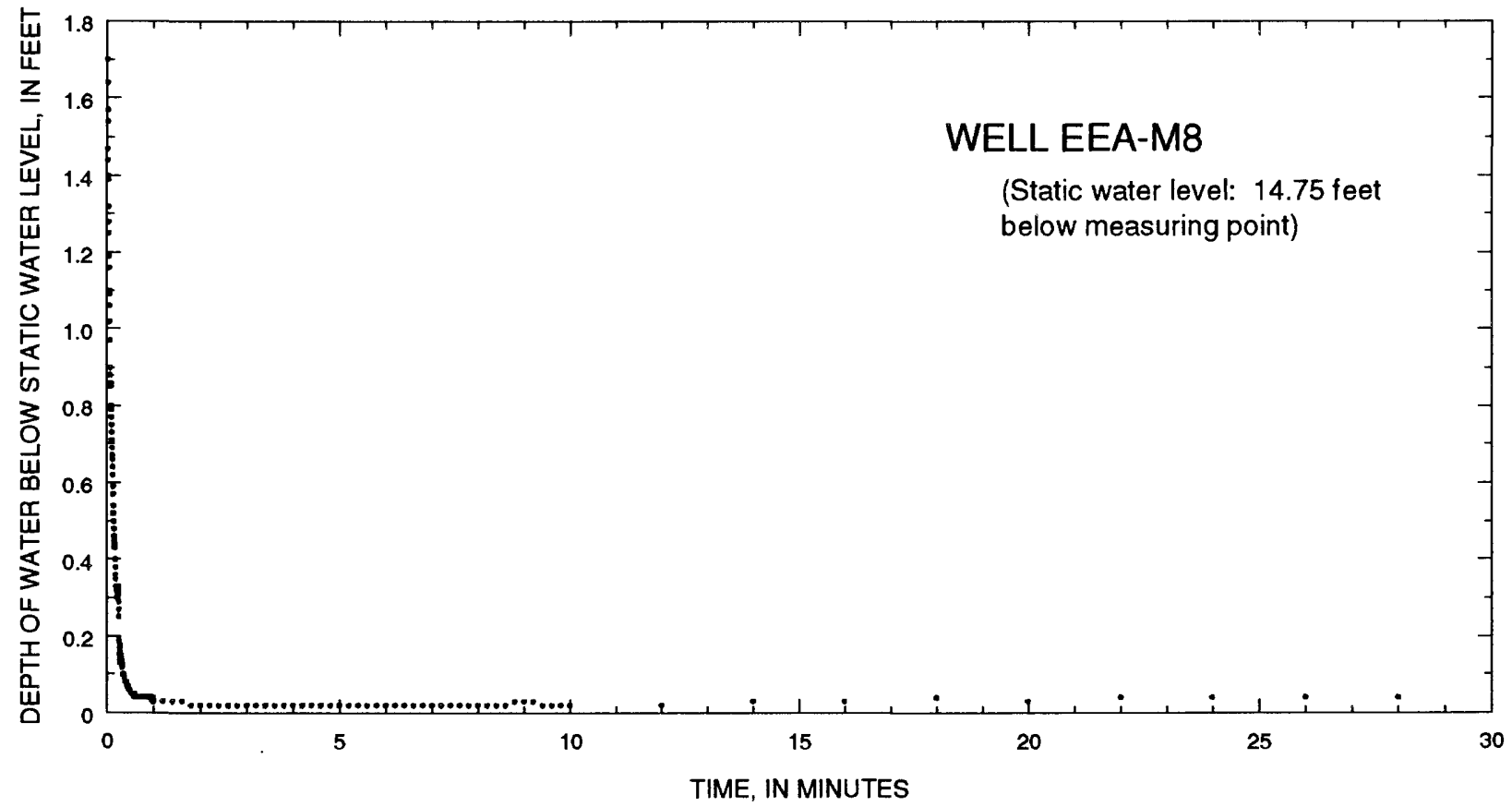

Figure 49. Rising-head data for slug test of observation well EEA-M8 at the Explosive Experimental Area, Naval Surface Warfare Center, Dahlgren Site, Dahlgren, Virginia. For location of observation well, see plate 1. 